\title{
Blow up for a Holonomic System
}

\author{
By
}

\author{
Orlando NeTO*
}

\begin{abstract}
We introduce a functor that associates to a holonomic system of microdifferential equations $\mathscr{M}$ on a contact manifold $X$ and a closed Lagrangian submanifold $\Lambda$ of $X$ a contact manifold $\tilde{X}$ and a holonomic system $\tilde{\mathscr{M}}$ on $\tilde{X}$. The manifold $\tilde{X}$ is an open set of the blow up of $X$ along a certain ideal of the sheaf of holomorphic functions on $X$. Moreover the restriction of $\mathscr{M}$ to the complementary of $\Lambda$ and the restriction of $\tilde{\mathscr{M}}$ to the complementary of the exceptional divisor of $\widetilde{X}$ are isomorphic as systems of microdifferential equations.
\end{abstract}

\section{§o. Introduction}

The structure of a regular holonomic system is well known at a generical point of the characteristic variety (cf. Kashiwara Kawai [11]). Nevertheless we know very little about it near a general singularity.

Hironaka proved a celebrated Theorem of resolution of singularities (cf. [7]). Roughly speaking it can be stated in the following way:

Given a complex manifold $X$ and a subvariety $Y$ of $X$ there is a new complex manifold $\tilde{X}$ and a holomorphic and bimeromorphic map $\pi: \widetilde{X} \rightarrow X$ with the following properties:

(i) If $S$ is the singular locus of $Y$ then the restriction of $\pi$ to $\pi^{-1}(X \backslash S)$ is a biholomorphic map onto $X \backslash S$.

(ii) The singularities of $\pi^{-1}(Y)$ are not "bad". The complex manifold $\tilde{X}$ is obtained by successively blowing up $X$ along convenient submanifolds.

The purpose of this paper is to built a notion of blow up for a holonomic system of microdifferential equations.

This should be a functor that associates to a holonomic $\mathcal{E}$-module $\mathscr{M}$

Communicated by M. Kashiwara, December 28, 1990.

1991 Mathematics Subject Classification: 58G15, 14E15, 53C15

* Departamento de Matematica, Universidade Classica de Lisboa, 1700 Lisboa Codex, Portugal. 
on a manifold $X$ and a certain submanifold $A$ of $X$ a holonomic $\varepsilon$-module $\tilde{\mathscr{M}}$ on a manifold $\tilde{X}$ with the following properties:

(i) There is a holomorphic bimeromorphic map $\pi: \widetilde{X} \rightarrow X$.

(ii) If $U,[\widetilde{U}]$ is an open set of $X[\tilde{X}]$ and $\pi$ induces a biholomorphic map from $\widetilde{U}$ onto $U$ then the microdifferential systems $\left.\mathscr{M}\right|_{U}$ and $\left.\tilde{\mathscr{M}}\right|_{\tilde{U}}$ are isomorphic.

(iii) The singularities of the support of $\tilde{\mathscr{M}}$ are never "worse" then the singularities of the support of $\mathscr{H}$. Moreover if the submanifold $\Lambda$ of $X$ was well chosen then the singularities of the support of $\tilde{\mathscr{M}}$ will be not as "bad" has the singularities of $\mathscr{M}$.

A first approach to the construction of this functor could be as follows. Let $X$ be a complex manifold, $\mathscr{M}$ an $\mathcal{E}_{X}$-module and let $\lambda$ be a point of $X$. Let $\pi: \widetilde{X} \rightarrow X$ be the blow up of $X$ along $\{\lambda\}$. The bimeromorphic map $\pi$ induces a bimeromorphic map $\hat{\pi}: T^{*} \tilde{X} \rightarrow T^{*} X$. The domain of $\hat{\pi}$ is $T^{*}(\tilde{X} \backslash E)$ where $E=\pi^{-1}(\{\lambda\})$ is the exceptional divisor of the blow up (cf. the end of this introduction). The domain of $\hat{\pi}$ is an open set of $T^{*} X$ and we did not get anything interesting.

Nevertheless if we consider the associated map $\tilde{\pi}: \mathbb{P}^{*}(\widetilde{X} \backslash E) \rightarrow \mathbb{P}^{*} X$ then we notice that there is a canonical extension $\tilde{\pi}$ of $\tilde{\pi}$ to $\mathbb{P}^{*} \tilde{X} \backslash \mathbb{P}_{E}^{*} \tilde{X}$. Here $\mathbb{P}^{*} \tilde{X}$ is the projective cotangent bundle of $\tilde{X}$ and $\mathbb{P}_{E}^{*} \tilde{X}$ is the projective conormal bundle of $\tilde{X}$ along $E$.

We remember that in [SKK] the sheaf of microdifferential operators was defined on the projective cotangent bundle.

There is a canonical isomorphism

$$
\left.\left.\widetilde{\boldsymbol{\pi}}^{-1} \mathcal{E}_{X}\right|_{\boldsymbol{P} *(\tilde{X} \backslash E)} \rightarrow \mathcal{E}_{\tilde{X}}\right|_{\boldsymbol{P} *(\tilde{X} \backslash E)} .
$$

There is a canonical sub ring $\mathcal{A}_{(1)}$ of $\mathcal{E}_{X}$ such that the restriction of (1) to $\mathcal{A}_{(1)}$ has a canonical extension to a morphism

$$
\Phi:\left.\widetilde{\boldsymbol{\pi}}^{-1} \mathcal{A}_{(1)} \rightarrow \mathcal{E}_{\tilde{X}}\right|_{\boldsymbol{P} * \tilde{X} \backslash P_{E}^{*} \tilde{X}}
$$

A holonomic $\mathcal{E}_{\widetilde{X}-\text {-module is }} \mathscr{A}_{(1)}$-coherent and therefore the $\left.\mathcal{E}_{\widetilde{X}}\right|_{\boldsymbol{P}^{*} \tilde{X} \backslash \boldsymbol{P}_{I}^{*} \tilde{X}^{-} \text {-module }}$

$$
\tilde{\mathscr{M}}=\left.\mathcal{E}_{\tilde{X}}\right|_{\boldsymbol{P} * \tilde{X} \backslash \boldsymbol{P}_{E}^{*} \tilde{X}} \otimes \mathcal{A}_{(1)} \mathscr{M}
$$

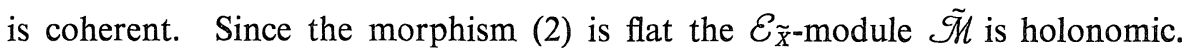
We call $\tilde{\mathscr{M}}$ the blow up of the $\mathcal{E}$-module $\mathscr{M}$ along $\Lambda$.

We need a microlocal version of the notion of blow up of an $\mathcal{E}$-module 
introduced above. In order to do that some problems must be solved.

First we have to study the blow up of a contact manifold along a Lagrangian submanifold. The reasons why we use contact manifolds instead of symplectic manifolds are the same why we use projective cotangent bundles instead of cotangent bundles in the construction above. Roughly speaking contact manifolds are the odd dimensional equivalent of homogeneous symplectic manifolds. For its definition cf. $\S 4$ or $[\mathrm{SKK}]$. We show in $\S 9$ that the blow up of a Lagrangian submanifold has a canonical structure of "contact manifold with logarithmic poles along its exceptional divisor". This generalization of the notion of contact manifold is introduced in $\S 4$. Sections 2 and 3 study the equivalent generalization of the notion of symplectic manifold, the notion of logarithmic symplectic manifold. In $\S 1$ we recall some basic facts on logarithmic differential forms.

We also have to quantize logarithmic symplectic manifolds.

In Chapter II we built and study sheaves of microdifferential operators on a logarithmic symplectic manifold. They are introduced in Sections 5 and 8. In Sections 6 and 7 we generalize the Division Theorems and results on quantized contact manifolds to the "logarithmic" case. In $\S 6$ we also study some special both side Ideals of the ring of microdifferential operators. This ideals are essential in the construction of the blow up.

Finally in Chapter III we generalize the construction discussed in the beginning of this Introduction. We present now a description in local coordinates of that construction. Let $X$ be a copy of $\mathbb{C}^{2}$ with coordinates $(x, y)$. Let $\lambda$ be the origin. The blow up $\tilde{X}$ of $X$ along $\{\lambda\}$ is the patching of two copies $X_{0}, X_{1}$ of $C^{2}$ with coordinates $\left(x, \frac{y}{x}\right),\left(\frac{x}{y}, y\right)$ by

$$
x=\frac{x}{y} y, \frac{y}{x}=\left(\frac{x}{y}\right)^{-1} \text {. }
$$

The restrictions of $\pi$ to $X_{0}, X_{1}$ are given respectively by

$$
x=x, y=x \frac{y}{x}, x=\frac{x}{y} y, y=y .
$$

If $E=\pi^{-1}(\lambda)$ is the exceptional divisor of $\tilde{X}$ then $E \cap X_{0}=\{x=0\}, E \cap X_{1}=\{y=$ $0\}$. Since the construction is symmetric on $x$ and $y$ we will from now on ignore $X_{1}$. Put $x_{0}=x, y_{0}=\frac{y}{x}$. Let $\left(x_{0}, y_{0}, \xi_{0}, \eta_{0}\right)$ be the canonical system of coordinates of $T^{*} X_{0}$ associated to $\left(x_{0}, y_{0}\right)$. The bimeromorphic map $\pi_{0}: T^{*} \tilde{X} \rightarrow T^{*} X$ 
is given by

$$
x=x_{0}, \quad y=x_{0} y_{0}, \quad \xi=\xi_{0}-\frac{y_{0}}{x_{0}} \eta_{0}, \quad \eta=\frac{1}{x_{0}} \eta_{0} .
$$

Its domain is therefore $\left\{x_{0} \neq 0\right\}$. We can understand (4) as a description of the bimeromorphic map $\tilde{\pi}: \mathbb{P}^{*} \tilde{X} \rightarrow \mathbb{P}^{*} X$ in homogeneous coordinates. If we multiply in $(4)(\xi, \eta)$ by $x$ we obtain another description of $\pi_{1}$.

$$
x=x_{0}, \quad y=x_{0} y_{0}, \quad \xi=x_{0} \xi_{0}+y_{0} \eta_{0}, \quad \eta=\eta_{0} .
$$

We conclude from $\left(4^{\prime}\right)$ that the domain of $\tilde{\pi}$ contains the complementary of $\left\{x_{0} \xi_{0}+y_{0} \eta_{0}=\eta_{0}=0\right\}$. This last set equals $\mathbb{P} \tilde{X}_{E} \tilde{X}$.

If

$$
\Phi:\left.\left.\tilde{\pi}^{-1} \mathcal{E}_{X}\right|_{\boldsymbol{P} *(\tilde{X} \backslash E)} \rightarrow \mathcal{E}_{\tilde{X}}\right|_{\boldsymbol{P} *(\tilde{X} \backslash E)}
$$

is the quantized contact transformation associated to the change of coordinates $x=x_{0}, y=x_{0} y_{0}$ then

$$
\begin{aligned}
\Phi(x) & =x_{0}, & \Phi\left(x \partial_{y}\right) & =\partial_{y_{0}}, \\
\Phi(y) & =x_{0} y_{0} & \Phi\left(\partial_{y}\left(x \partial_{x}+y \partial_{y}\right)\right) & =\partial_{x_{0}} \partial_{y_{0}} .
\end{aligned}
$$

If $\mathcal{A}_{(1)}$ is the sub $\mathcal{E}_{X}$-algebra of $\mathcal{E}_{X}$ generated by $x \partial_{y}$ and $\partial_{x}\left(x \partial_{x}+y \partial_{y}\right)$ then there is an extension of $\left.\Phi\right|_{\pi} ^{-1} \mathcal{A}_{(1)}$ to a morphism

$$
\tilde{\Phi}:\left.\tilde{\pi}^{-1} \mathcal{A}_{(1)} \rightarrow \mathcal{E}_{\tilde{X}}\right|_{\boldsymbol{P} * \tilde{X} \backslash \boldsymbol{P}_{E}^{*} \tilde{X}}
$$

\section{Contents}

§. Introduction

\section{Chapter I}

$\S 1$. Logarithmic differential forms

$\S 2$. Logarithmic symplectic manifolds

$\S 3$. Homogeneous logarithmic symplectic manifolds

\$4. Logarithmic contact manifolds

Chapter II

$\S 5$. Logarithmic microdifferential operators

§6. Division Theorems

§7. Quanitzed contact transformations

§8. Quantized logarithmic contact manifolds 


\section{Chapter III}

§. Blow up of a quantized contact manifold along a Lagrangian submanifold

$\S 10$. Blow up of a logarithmic contact manifold along its residual submanifold

$\S 11$. Total blow up of a logarithmic contact manifold along a Lagrangian submanifold

\section{Chapter I. Logarithmic Symplectic Manifolds}

In this Chapter we introduce generalizations of the notions of symplectic manifold, homogeneous symplectic manifold and contact manifold. We allow the differential forms involved in the definitions of the concepts defined above to have logarithmic poles along a fixed divisor with normal crossings.

These manifolds have properties very similar to the ones they generalize. For instance, it is still possible to prove Darboux type Theorems in this context. The local model of a logarithmic symplectic manifold is still a vector bundle, the vector bundle $\pi: T^{*}\langle X \mid Y\rangle \rightarrow X$ whose sheaf of sections is the locally free $\mathcal{O}_{X}$-module $\Omega_{X}^{1}\langle Y\rangle$ of logarithmic differential forms of $X$ with poles along a divisor with normal crossings $Y$.

There is a canonical differential form $\theta$ of degree 1 on $T^{*}\langle X \mid Y\rangle$ with logarithmic poles along $\pi^{-1}(Y)$ (cf. $\S 1$.). Suppose that $\left(x_{1}, \cdots, x_{n}\right)$ is a system of local coordinates on a open set $U$ of $X$ such that $Y \cap U=\left\{x_{1}=0\right\}$. Then there is a system of local coordinates $\left(x_{1}, \cdots, x_{n}, \xi_{1}, \cdots, \xi_{n}\right)$ in $\pi^{-1}(U)$ such that

$$
\left.\theta\right|_{\pi^{-1}(U)}=\xi_{1} \frac{d x_{1}}{x_{1}}+\xi_{2} d x_{2}+\cdots+\xi_{n} d x_{n} .
$$

The subsets $\left\{x_{1}=0\right\}$ and $\left\{x_{1}=\xi_{1}=0\right\}$ are invariantly defined. They are called respectively the set of poles and the residual submanifold of $T^{*}\langle X \mid Y\rangle$. The existence of the residual submanifold is the main new phenomena that we find in this generalization of the notion of symplectic manifold. For instance, the residual submanifold contains involutive subsets of codimension superior to the dimension of $X$. Moreover it has a canonical structure of symplectic manifold. This two submanifolds will be essential in the construction of the blow up.

\section{§1. Logarithmic Differential Forms}

Let $X$ be a complex manifold. A subset $Y$ of $X$ is called a divisor with 
normal crossings if for any $x^{0} \in Y$ there is an open neighbourhood $U$ of $x^{0}$, a system of local coordinates $\left(x_{1}, \cdots, x_{n}\right)$ defined on $U$ and an integer $\nu$ such that

$$
Y \cap U=\left\{x_{1} \cdots x_{\vartheta}=0\right\} .
$$

Fixed a divisor with normal crossings $Y$ and an open subset $U$ of $X$ let $j: U_{0}=$ $U \backslash Y \hookrightarrow U$ be the inclusion map. Let $f$ be a holomorphic function defined on $U$. If the set of zeros of $f$ is contained in $Y \cap U$ then we denote by $\delta f$ the global section $d f / f$ of $j_{*} \Omega_{U_{0}}^{1}$. Otherwise $\delta f$ will denote the differential $d f$ of $f$.

We remark that the correspondence $f \mapsto \delta f$ is not a morphism of sheaves.

Let $\Omega_{X}^{*}\langle Y\rangle$ be the smallest subcomplex of $j_{*} \Omega_{X_{0}}^{*}$ stable by exterior product and containing $\mathcal{O}_{X}$ and $\delta f$ whenever $f$ is a local section of $\mathcal{O}_{X}$. The local sections of $\Omega_{X}^{*}\langle Y\rangle$ are called logarithmic differential forms with poles along $Y$.

Let $\Theta_{X}$ be the sheaf of vector fields of $X$. Let $I_{Y}$ be the defining Ideal of $Y$, that is, the Ideal of the local sections of $\mathcal{O}_{X}$ that vanish along $Y$. We say that a vector field $u$ of $X$ is tangent to $Y$ if $u I_{Y} \subset I_{Y}$. Let $\Theta_{X}\langle Y\rangle$ be the sheaf of the vector fields of $X$ that are tangent to $Y$.

The $\mathcal{O}_{X}$-modules $\Omega_{X}^{1}\langle Y\rangle$ and $\Theta_{X}\langle Y\rangle$ are locally free and dual of each other. Given an open set $U$ and a system of local coordinates $\left(x_{1}, \cdots, x_{n}\right)$ on $U$ verifying (1.0.1) we will denote by $\left(\delta_{x_{i}}\right)$ or $\left(\frac{\delta}{\delta x_{i}}\right)$ the dual basis of $\left(\delta x_{i}\right)$.

We notice that if moreover $x_{i}$ vanishes at some point of $U$ for $\nu+1 \leq i \leq n$ then

$$
\begin{array}{lll}
\delta x_{i}=\frac{d x_{i}}{x_{i}}, & \delta_{x_{i}}=x_{i} \partial_{x_{i}} & \text { for } \quad 1 \leq i \leq \nu, \\
\delta x_{i}=d x_{i}, & \delta_{x_{i}}=\partial_{x_{i}} & \text { for } \nu+1 \leq l \leq n .
\end{array}
$$

Let $W_{m}\left(\Omega_{X}^{*}\right)\langle Y\rangle$ be the smallest sub $\mathcal{O}_{X}$-module of $\Omega_{X}^{*}\langle Y\rangle$ stable by exterior product and containing $\delta f_{1} \cdots \delta f_{l}$ whenever $\left(f_{1}, \cdots, f_{l}\right)$ is local section of $\mathcal{O}_{X}^{l}$ and $l \leq m$. The $\mathcal{O}_{X}$-modules $\left(W_{m}\right)$ constitute an increasing filtration of $\Omega_{X}\langle Y\rangle$ by subcomplexes. We will denote by $W_{m}\left(\Omega_{X}^{k}\langle Y\rangle\right)$ the sheaf of sections of $W_{m}\left(\Omega_{X}^{*}\langle Y\rangle\right)$ of degree $k$.

For $1 \leq l \leq \nu$ put $Y_{l}=\left\{x_{l}=0\right\}$. The set $Y_{l}$ is a closed submanifold of $U$ and an irreducible component of $Y \cap U$. If $1 \leq l_{1} \leq \cdots \leq l_{k} \leq \nu$ we put $Y_{l_{1}, \cdots, l_{k}}=$ $Y_{l_{1}} \cap \cdots \cap Y_{l_{k}}$. If $0 \leq m \leq p$ the support of the sheaf $G_{m}^{W}\left(\Omega_{U}^{p}\langle Y \cap U\rangle\right)$ is the union of the submanifolds $Y_{l_{1}, \cdots, l_{m}}$. Otherwise the sheaf $\operatorname{Gr}_{m}^{W}\left(\Omega_{U}^{p}\langle Y \cap U\rangle\right)$ vanishes.

Given integers $1 \leq l_{1} \leq \cdots \leq l_{m} \leq \nu$ we can define a morphism of sheaves 


$$
\operatorname{Res}_{Y_{l_{1}}, \cdots, l_{m}}: G r_{m}^{W}\left(\Omega_{U}^{p}\langle Y \cap U\rangle\right) \rightarrow \Omega_{Y}^{p-m}, \cdots, l_{m}
$$

in the following way: If $\left\{j_{1}, \cdots, j_{p}\right\} \cap\left\{l_{l}, \cdots, l_{m}\right\}=\phi$ and $f$ is a local section of $\mathcal{O}_{U}$ then

$$
\operatorname{Res}_{Y_{l_{1}, \cdots, l_{m}}}\left(f d x_{j_{1}} \cdots d x_{j_{p}} \delta x_{l_{1}} \cdots \delta x_{l_{m}}\right)=\left.f\right|_{Y_{l_{1}, \cdots, l_{m}}} d x_{j_{1}} \cdots d x_{j_{p}} .
$$

We call the differential form $\operatorname{Res}_{Y_{l_{1}}, \cdots, l_{m}} \alpha$ the Poincaré residue of $\alpha$ along $Y_{l_{1}, \cdots, l_{m}}$. For a global construction of the Poincaré residue cf. [2].

\section{§2. Logarithmic Symplectic Manifolds}

In this paper all the vector spaces will be over the field of complex numbers.

Let $E$ be a vector space of dimension $2 n$ and $\sigma$ a symplectic form on $E$. Let

$$
e_{j}, j \in A, f_{k}, k \in B,
$$

where $A, B \subset\{1, \cdots, n\}$, be a family of vectors of $E$. We say that (2.0.1) is a partial symplectic basis for $\sigma$ if

$$
\sigma\left(f_{j}, e_{k}\right)=\delta_{j k}, \sigma\left(f_{i}, f_{j}\right)=\sigma\left(e_{k}, e_{l}\right)=0 \quad i, j \in A, k, l \in B .
$$

If $A=B=\{1, \cdots, n\}$ then (2.0.1) is called a symplectic basis for $\sigma$.

The symplectic form $\sigma$ defines an isomorphism $H$ from the dual $E^{\prime}$ of $E$ onto $E$ in the following way: given a linear form $\alpha$ on $E$ then $H(\alpha)$ is the only vector of $E$ such that

$$
\langle u, \alpha\rangle=\sigma(u, H(\alpha)), \quad u \in E .
$$

One calls $H(\alpha)$ the Hamiltonian vector of $\alpha$.

(2.0.2) We notice that the isomorphism $H$ determines $\sigma$. Moreover the isomorphism $H$ defines a symplectic form $\{\star, \star\}$ on $E^{\prime}$ by

$$
\{\alpha, \beta\}=\sigma(H(\alpha), H(\beta)) \quad \alpha, \beta \in E^{\prime} .
$$

Definition 2.1. Let $X$ be a complex manifold an $Y$ a divisor with normal crossings of $X$. Let

$$
\pi: T^{*}\langle X \mid Y\rangle \rightarrow X
$$

be the vector bundle with sheaf of sections $\Omega_{X}^{1}\langle Y\rangle$. We will call (2.1.1) the logarithmic cotangent bundle of $X$ along $Y$. Let

$$
\tau: T\langle X \mid Y\rangle \rightarrow X
$$


be the vector bundle with sheaf of sections $\Theta_{X}\langle Y\rangle$. We call (2.1.2) the logarith mic tangent bundle of $X$ along $Y$.

Remark 2.2. Given a section $\alpha$ of $\Omega_{X}^{1}\left(\Theta_{X}\right)$ we will represent its value at $x^{0} \in X$ as a section of $\Omega_{X}^{1}\left(\Theta_{X}\right)$ by $\alpha_{\left(x^{0}\right)} \in T_{x 0}^{*} X\left(\in T_{x^{0}} X\right)$. Given a section $\alpha$ of $\Omega_{X}^{1}\langle Y\rangle\left(\Theta_{X}\langle Y\rangle\right)$ we will represent its value at $x^{0} \in X$ as a section of $\Omega_{X}^{1}\langle Y\rangle$ $\left(\Theta_{X}\langle Y\rangle\right)$ by $\alpha_{\left\langle x^{0}\right\rangle} \in T_{x 0}^{*}\langle X \mid Y\rangle\left(T_{x^{0}}\langle X / Y\rangle\right)$.

Definition 2.3. Let $X$ be a complex manifold and $Y$ a divisor with normal crossings of $X$. We say that a locally exact section $\sigma$ of $\Omega_{X}^{2}\langle Y\rangle$ is a logarithmic symplectic form with poles along $Y$ if $\sigma_{\left\langle x^{0}\right\rangle}$ is a symplectic form on $T_{x^{0}}\langle X \mid Y\rangle$ for any $x^{0} \in X$.

We say that a complex manifold $X$ with a logarithmic symplectic form along a divisor with normal crossings $Y$ of $X$ is a logarithmic symplectic manifold with poles along $Y$. If $X_{1}, X_{2}$ are logarithmic symplectic manifolds with logarithmic symplectic forms $\sigma_{1}, \sigma_{2}$ and $\varphi$ is a holomorphic map form $X_{1}$ into $X_{2}$ such that $\varphi^{*} \sigma_{2}=\sigma_{1}$ then $\varphi$ is called a morphism of logarithmic symplectic manifolds. If moreover $\varphi$ is biholomorphic we say that $\varphi$ is an isomorphism of logarithmic symplectic manifolds or a canonical transformation.

Remark 2.4. (i) If $Y$ is the empty set we get the usual definition of symplectic manifold.

(ii) A logarithmic symplectic manifold has always even dimension.

(iii) Suppose that $X$ has dimension $2 n$. A locally exact section $\sigma$ of $\Omega_{X}^{2}\langle Y\rangle$ is a logarithmic symplectic form with poles along $Y$ if and only if $\sigma^{n}$ is a generator of $\Omega_{X}^{2 n}\langle Y\rangle$.

(iv) We notice that a morphism of logarithmic symplectic manifolds is not necessarily a local homeomorphism (cf. Remark 10.3.).

The Hamiltonian isomorphisms $H_{x 0}: T_{x 0}^{*}\langle X \mid Y\rangle \rightarrow T_{x 0}\langle X \mid Y\rangle$ induce an isomorphism of $\mathcal{O}_{X}$-modules

$$
H: \Omega_{X}^{1}\langle Y\rangle \rightarrow \Theta_{X}\langle Y\rangle .
$$

If $\alpha$ is a local section of $\Omega_{X}^{1}\langle Y\rangle$ then $H(\alpha)$ is the only local section $u$ of $\Theta_{X}\langle Y\rangle$ such that $\iota(u) \sigma=\alpha$, where $\iota(u) \sigma$ is the interior product of $u$ and $\sigma$. We notice that

$$
\left\{\alpha_{\left\langle x^{0}\right\rangle}, \beta_{\left\langle x^{0}\right\rangle}\right\}_{x^{0}}=\{\alpha, \beta\}\left(x^{0}\right),
$$

where $\left\{\hbar,{ }^{2}\right\}_{x^{0}}$ is the canonical symplectic form of $T_{x 0}^{*}\langle X / Y\rangle$.

Definition 2.5. Given a complex manifold $X$ we say that a $\mathbb{C}$-bilinear mor- 
phism

$$
\{\star, \star\}: \mathcal{O}_{X} \times \mathcal{O}_{X} \rightarrow \mathcal{O}_{X}
$$

is a Poisson bracket if it verifies the following conditions:

(i) $\{f, g\}=-\{g, f\}$

(ii) $\{f g, h\}=f\{g, h\}+g\{f, h\}$

(iii) $\{\{f, g\}, h\}+\{\{g, h\}, f\}+\{\{h, f\}, g\}=0$.

We call $\{f, g\}$ the Poisson bracket of $f$ and $g$.

If $f$ is a local section of $\mathcal{O}_{X}$ the derivation $g \mapsto\{f, g\}$ determines a vector field $H_{f}$, the Hamiltonian vector field of $f$.

We call a complex manifold $X$ endowed with a Poisson bracket a Poisson manifold.

If $\left(X_{1},\{\star, \star\}_{1}\right),\left(X_{2},\{\star, \star\}_{2}\right)$ are Poisson manifolds and $\varphi: X_{1} \rightarrow X_{2}$ is a complex map such that $\left\{\varphi^{*} f, \varphi^{*} g\right\}_{1}=\varphi^{*}\{f, g\}_{2}$, for any holomorphism functions $f, g$ defined in an open set of $X_{2}$ we call $f$ a morphism of Poisson manifolds.

Example 2.6. A logarithmic symplectic manifold has a canonical structure of Poisson manifold. Actually the bilinear form

$$
(f, g) \mapsto H(d f)(g)
$$

is a Poisson bracket on $\mathcal{O}_{X}$.

Definition 2.7. Let $X$ be a Poisson manifold. An analytical subset $V$ of $X$ is called involutive if $\left\{I_{V}, I_{V}\right\} \subset I_{V}$.

Proposition 2.8. Let $\sigma$ be a logarithmic symplectic form on a symplectic manifold $X$. Then we can recover $\sigma$ from the Poisson bracket it determines.

Proof. By (2.1.2) it is enough to show that, given $x^{0} \in X$ we can recover the Hamiltonian isomorphism $H_{x 0}: T_{x 0}^{*}\langle X \mid T\rangle \rightarrow T_{x 0}\langle X \mid Y\rangle$ from the Poisson bracket of $\mathcal{O}_{X}$. This can easily be acomplished once we fix a system of coordinates in an open neighbourhood of $x^{0}$ verifying (1.0.1).

Q.E.D.

Corollary 2.9. Let $X_{1}, X_{2}$ be logarithmic complex manifolds and $\varphi$ a biholomorphic map from $X_{1}$ onto $X_{2}$. Then $\varphi$ is a canonical transformation if and only if it is a morphism of Poisson manifolds.

Example 2.10. If $X$ is a complex manifold and $Y$ a divisor with normal crossings of $X$ then the vector bundle $\pi: T^{*}\langle X \mid Y\rangle \rightarrow X$ has a canonical struc- 
ture of logarithmic symplectic manifold with poles along $\pi^{-1} Y$.

For $i=1,2$ let $Y_{i}$ be a divisor with normal crossings of a complex manifold $X_{i}$. If $f: X_{1} \rightarrow X_{2}$ is a holomorphic map such that $f^{-1} Y_{2}=Y_{1}$ then we have a canonical morphism of vector bundles

$$
\rho_{f}: X_{1} \times_{X_{2}} T^{*}\left\langle X_{2} / Y_{2}\right\rangle \rightarrow T^{*}\left\langle X_{1} / Y_{1}\right\rangle
$$

defined in the following way: if $\alpha$ is a local section of $\Omega_{X_{2}}^{1}\left\langle Y_{2}\right\rangle$ then $\rho_{f}(\alpha)=$ $f^{*} \alpha$. The composition of $\rho_{\pi}$ with the diagonal map

$$
T^{*}\langle X \mid Y\rangle \rightarrow T^{*}\langle X \mid Y\rangle \times_{X} T^{*}\langle X \mid Y\rangle
$$

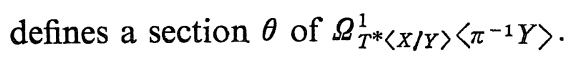

We call $\theta$ the canonical 1 -form of $T^{*}\langle X / Y\rangle$.

Given an integer $\nu$ and a system of local coordinates $\left(x_{1}, \cdots, x_{n}\right)$ on an open set $U$ of $X$ verifying (1.0.1), there is one and only one family of holomorphic functions $\xi_{i}, 1 \leq i \leq n$, defined on $\pi^{-1}(U)$ such that

$$
\left.\theta\right|_{\pi^{-1}(U)}=\sum_{i=1}^{\nu} \xi_{i} \frac{d x_{i}}{x_{i}}+\sum_{i=\nu+1}^{n} \xi_{i} d x_{i} .
$$

The functions

$$
x_{1}, \cdots, x_{n}, \xi_{1}, \cdots, \xi_{n}
$$

define a system of local coordinates on $\pi^{-1}(U)$, called the system of canonical coordinates with poles along $Y$ associated to the system of local coordinates $\left(x_{1}, \cdots, x_{n}\right)$.

Remark 2.10.1. We notice that $\xi_{1}, \cdots, \xi_{n}$ depend not only of $\left(x_{1}, \cdots, x_{n}\right)$ but also of $\nu$ and $U$. Nevertheless there is one and only one $\nu$ verifying the additional condition " $x_{i}$ vanishes at some point of $U$ for $\nu+1 \leq i \leq n$ ". Moreover if we fix $\nu$ then $\xi_{1}, \cdots, \xi_{n}$ will not depend of the open subset of $U$ we choose.

The 2-form $\sigma=d \theta$ is called the canonical 2-form of $T^{*}\langle X \mid Y\rangle$. The canonical 2-form is a sympletic form with poles along $\pi^{-1} Y$.

Given holomorphic functions $f, g$, defined on an open set $V$ contained in $\pi^{-1} U$, we have

$$
\{f, g\}=\sum_{i=1}^{\nu} x_{i}\left(\frac{\partial f}{\partial \xi_{i}} \frac{\partial g}{\partial x_{i}}-\frac{\partial f}{\partial x_{i}} \frac{\partial g}{\partial \xi_{i}}\right)+\sum_{i=\nu+1}^{n}\left(\frac{\partial f}{\partial \xi_{i}} \frac{\partial g}{\partial x_{i}}-\frac{\partial f}{\partial x_{i}} \frac{\partial g}{\partial \xi_{i}}\right) .
$$

In particular

$$
\left\{\xi_{i}, x_{j}\right\}= \begin{cases}\delta_{i j} x_{j} & \text { if } 1 \leq j \leq \nu \\ \delta_{i j} & \text { if } \nu+1 \leq j \leq n\end{cases}
$$


If $1 \leq i \leq \nu$ then

$$
\left.d \xi_{i}\right|_{\left\{x_{i}=0\right\}}=d \operatorname{Res}_{\left\{x_{i}=0\right\}} \sigma, \quad 1 \leq i \leq \nu .
$$

We will now show that any logarithmic symplectic manifold is locally isomorphic to $T^{*}\left\langle\boldsymbol{C}^{n} /\left\{x_{1} \cdots x_{\nu}=0\right\}\right\rangle$ for some integer $\nu$.

Definition 2.11. Let $(X, \sigma)$ be a logarithmic symplectic manifold with poles along a divisor with normal crossings $Y$. Let $U$ be an open set of $X$ and $Y_{0}$ a global smooth hypersurface contained in $Y \cap U$. A holomorphic function $\xi$ defined on $U$ is called a residual function along $Y_{0}$ if

$$
\left.d \xi\right|_{Y_{0}}=d \operatorname{Res}_{Y_{0}}\left(\left.\sigma\right|_{U}\right) .
$$

Remark 2.11.1. If $\xi, \eta$ are residual functions along $Y_{0}$ then there is a constant $\lambda$ such that $\xi-\eta-\lambda \in I_{Y_{0}}$.

Proposition 2.12. Let $(X, \sigma)$ be a logarithmic symplectic manifold with poles along a divisor with normal crossings $Y$. Let $x^{0}$ be a point of $X$. Let $x_{1}, \cdots, x_{v}, \xi_{1}, \cdots, \xi_{v}$ be holomorphic functions defined in an open neighbourhood $V$ of $x^{0}$ such that $Y \cap V=\left\{x_{1} \cdots x_{\nu}=0\right\}, d x_{1} \cdots d x_{\nu}$ does not vanish along $Y \cap V$ and $\xi_{i}$ is a residual function along $\left\{x_{i}=0\right\}$ for $1 \leq i \leq \nu$.

Then there is an open neighbourhood $U$ of $x^{0}$ and a differential form $\alpha$ of degree 2 such that

$$
\left.\sigma\right|_{U}=\sum_{i=1}^{\nu} d \xi_{i} \delta x_{i}+\alpha
$$

Proof. There are holomorphic functions $\eta_{i}, 1 \leq i \leq \nu$, and a differential form $\beta$ of degree 1, defined in a neighbourhood $U$ of $x^{0}$ such that

$$
d\left(\sum_{i=1}^{\nu} \eta_{i} \delta x_{i}+\beta\right)=\sigma .
$$

The functions $\eta_{i}$ are residual functions along $\left\{x_{i}=0\right\}$. By Remark 2.11.1 there are constants $\lambda_{i}$ and holomorphic functions $f_{i}$ such that

$$
\sum_{i=1}^{\nu} \eta_{i} \delta x_{i}+\beta=\sum_{i=1}^{\nu} \xi_{i} \delta x_{i}+\beta+\sum_{i=1}^{\nu} f_{i} d x_{i}+\sum_{i=1}^{\nu} \lambda_{i} \delta x_{i} . \quad \text { Q.E.D. }
$$

Corollary 2.13. Let $(X, \sigma)$ be a logarithmic symplectic manifold of dimension $2 n$ with poles along a divisor with normal crossings $Y$. Then the number of irreducible components of $Y$ at $x^{0}$ is smaller or equal to $n$ at any point $x^{0}$ of $Y$.

Proof. We will use the notations of Proposition 2.12. We fix $x^{0} \in Y$. Let $\nu$ be the number of irreducible components of $Y$ at $x^{0}$. Then by (2.12.3) there is a constant $C$ such that 


$$
\sigma^{n} \equiv C d \xi_{1} \cdots d \xi_{\nu} \delta x_{1} \cdots \delta x_{\nu} \alpha^{n-\nu} \quad\left(\bmod W_{\nu-1} \Omega_{X}^{2 n}\langle Y\rangle\right)
$$

Therefore the residue of $d \xi_{1} \cdots d \xi_{y} \delta x_{1} \cdots \delta x_{y}$ along $\left\{x_{1}=\cdots=x_{\nu}=0\right\}$ does not vanish at $x^{0}$. Hence

$$
d \xi_{1} \cdots d \xi_{\nu} d x_{1} \cdots d x_{v}\left(x^{0}\right) \neq 0 .
$$

Corollary 2.14. Let $(X, \sigma)$ be a logarithmic symplectic manifold with poles along a divisor with normal crossings $Y$. Let $\left(x_{1}, \cdots, x_{n}, \xi_{1}, \cdots, \xi_{n}\right)$ be a system of local coordinates in an open neighbourhood $V$ of $X$ such that $x_{1}, \cdots$, $x_{y}, \xi_{1}, \cdots, \xi_{y}$ verify the conditions of Proposition 2.12. Then there are local sections $u_{i}$, $v_{i}$ of $\Theta_{X}\langle Y\rangle$ such that

$$
\frac{1}{x_{i}} H_{\xi_{i}}=\frac{\partial}{\partial x_{i}}+u_{i}, \quad H\left(\delta x_{i}\right)=-\frac{\partial}{\partial \xi_{i}}+v_{i} .
$$

Proof. For $1 \leq i \leq \nu$ there are differential forms $\beta_{i}, \gamma_{i}$ of degree 1 such that:

$$
\iota\left(x_{i} \frac{\partial}{\partial x_{i}}\right) \sigma=d \xi_{i}-x_{i} \beta_{i}, \quad \iota\left(-x_{i} \frac{\partial}{\partial \xi_{i}}\right) \sigma=d x_{i}-x_{i} \gamma_{i}
$$

Therefore

$$
\begin{aligned}
& H_{\xi_{i}}=x_{i} \frac{\partial}{\partial x_{i}}+x_{i} H\left(\beta_{i}\right), \\
& H_{x_{i}}=-x_{i} \frac{\partial}{\partial \xi_{i}}+x_{i} H\left(\gamma_{i}\right) .
\end{aligned}
$$

Remark 2.15. It follows from Proposition 2.14 that, with the notations of Proposition 2.12, the following relations hold:

(i) There are holomorphic functions $f_{i j}, 1 \leq i, j \leq \nu$, such that

$$
\left\{\xi_{i}, x_{j}\right\}=\delta_{i j} x_{j}+x_{i} x_{j} f_{i j} .
$$

(ii) Given a holomorphic function $f$ the functions $\left\{\xi_{i}, f\right\},\left\{x_{i}, f\right\}$ vanish along $\left\{x_{i}=0\right\}$.

(iii) For $1 \leq i, j \leq \nu\left\{d \xi_{i}, \delta x_{j}\right\}_{x^{0}}=\delta_{i j}$.

(iv) For any differential form $\alpha$ of degree $1\left\{d \xi_{i}, \alpha\right\}_{x^{0}}=0$.

Definition 2.16. Let $(X, \sigma)$ be a logarithmic symplectic manifold of dimension $2 n$ with poles along a divisor with normal crossings $Y$. Let $U$ be an open set of $X$ and let $A, B$ be subsets of $\{1, \cdots, n\}$. A family of holomorphic functions 


$$
x_{j}, j \in A, \quad \xi_{k}, k \in B
$$

defined on $U$ is called a partial system of logarithmic symplectic coordinates for $(X, \sigma)$ on $U$ if it verifies the following conditions:

(i) There is an integer $\nu$ such that $\{1, \cdots, \nu\}$ is contained in $A$ and $Y \cap U=$ $\left\{x_{1} \cdots x_{\nu}=0\right\}$.

(ii) The holomorphic function $\xi_{k}$ is a residual function along $\left\{x_{k}=0\right\}$, for $1 \leq k \leq \nu, k \in B$.

(ii) For $i, j \in A, k, l \in B$

$$
\left\{\xi_{i}, x_{j}\right\}=\left\{\begin{array}{lll}
\delta_{i j} x_{j} & \text { if } \quad 1 \leq j \leq \nu ; \\
\delta_{i j} & \text { if } \quad \nu+1 \leq j \leq n
\end{array}\right.
$$

and

(iv) The vectors

$$
\left\{\xi_{k}, \xi_{l}\right\}=\left\{x_{i}, x_{j}\right\}=0
$$

$$
\delta x_{j}, j \in A, d \xi_{k}, k \in B,
$$

constitute a partial symplectic basis of $T_{x 0}^{*}\langle X \mid Y\rangle$.

A partial system of logarithmic symplectic coordinates is called a system of logarithmic symplectic coordinates if $A=B=\{1, \cdots, n\}$.

Theorem 2.17. Let $X$ be a logarithmic symplectic manifold of dimension $2 n$ with poles along a divisor with normal crossings $Y$. Given $x^{0} \in X$ and a partial system of symplectic local coordinates in a neighbourhood of $x^{0}$ there is a system of logarithmic symplectic coordinates that extends the partial system above.

We will first notice some properties of the vector space $T_{x 0}^{*}\langle X \mid Y\rangle$.

Lemma 2.18. (i) The choice of a family of functions $x_{1}, \cdots, x_{\nu}$ defined in an open neighbourhood $U$ of $x^{0}$, vanishing at $x^{0}$ and verifying (1.0.1) determines a supplement of the subspace $\operatorname{Im}\left(T_{x 0}^{*} X \rightarrow T_{x 0}^{*}\langle X \mid Y\rangle\right)$ of $T_{x 0}^{*}\langle X \mid Y\rangle$, the span of $\delta x_{i\left\langle x^{0}\right\rangle}, 1 \leq i \leq \nu$.

(ii) Given a residual function along $\left\{x_{i}=0\right\} \xi_{i}$ the vector $r_{i}=d \xi_{i\left\langle x^{0}\right\rangle}$ does not depend of the choice of the function $\xi_{i}$.

(iii) The span of

$$
\delta x_{1}, \cdots, \delta x_{v}, r_{1}, \cdots, r_{\nu}
$$

is a symplectic vector subspace of $T_{x 0}^{*}\langle X \mid Y\rangle$ that admits (2.18.2) as a symplectic basis. Moreover the vectors $r_{1}, \cdots, r_{\nu}$ are in the symplectic orthogonal of $\operatorname{Im}\left(T_{x 0}^{*} X \rightarrow T_{x 0}^{*}\langle X \mid Y\rangle\right)$. 
(iv) Given the partial symplectic basis (2.16.2) there is a symplectic basis of $T_{x 0}^{*}\langle X \mid Y\rangle$

$$
e_{j}, f_{k}, 1 \leq j, k \leq n,
$$

such that $e_{j}=\delta x_{j}\left\langle x^{0}\right\rangle, j \in A, f_{k}=d \xi_{k\left\langle x^{0}\right\rangle}, k \in B, f_{k}=r_{k}, 1 \leq k \leq \nu$ and $e_{j}, f_{k} \in$ $\operatorname{Im}\left(T_{x 0}^{*} X \rightarrow T_{x 0}^{*}\langle X \mid Y\rangle\right)$ for $j, k \geq \nu+1$.

Proof. There is one and only one linear map

$$
T_{x 0}^{*}\left\{x_{i}=0\right\} \rightarrow T_{x 0}^{*}\langle X \mid Y\rangle
$$

such that the diagram bellow commutes.

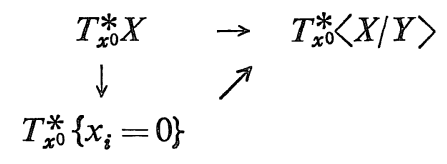

The vector $r_{i}$ is the image of $\operatorname{Res}_{\left\{x_{i}=0\right\}} \sigma\left(x^{0}\right)$ by the map (2.18.1). This proves (ii). Statement (iii) is a straightforward consequence of Remark 2.15. Finally (iv) lollows from (iii).

Q.E.D.

2.19. We will now prove Theorem 2.17.

Given the partial system of logarithmic symplectic local coordinates (2.16.2) there is a symplectic basis (2.18.3) verifying the conditions of (iv) and holomorphic functions

$$
q_{1}, \cdots, q_{n}, p_{1}, \cdots, p_{n}
$$

such that, for $1 \leq j, k \leq n, q_{j}=x_{j}, j \in A, p_{k}=\xi_{k}, k \in B ; d q_{j_{\left\langle x^{0}\right\rangle}}=e_{j}, d p_{k_{\left\langle x^{0}\right\rangle}}=f_{k}$ and moreover $p_{k}$ is a residual function along $\left\{x_{k}=0\right\}$ for $1 \leq k \leq \nu$. The functions (2.18.4) constitute a system of local coordinates for $X^{0}$ in some neighbourhood of $x^{0}$. Actually

$$
d p_{1} \cdots d p_{n} \frac{d q_{1}}{q_{1}} \cdots \frac{d q_{v}}{q_{v}} d q_{v+1} \cdots d q_{n}
$$

constitute a local generator of $\Omega_{X}^{2 n}\langle Y\rangle$ in some neighbourhood of $x^{0}$.

Suppose that there is $k_{0} \notin B$ such that $k_{0} \geq \nu+1$. Consider the system of equations

$$
H\left(\delta x_{j}\right) \xi_{k_{0}}=-\delta_{k_{0} j}, j \in A, H_{\xi_{k}} \xi_{k_{0}}=0, k \in B .
$$

We conclude from Corollary 2.14. that the vector fields $\left(\left(1 / x_{k}\right) H_{p_{k}}\right)\left(x^{0}\right), 1 \leq$ $k \leq \nu$, span a supplement of $\operatorname{Im}\left(T_{x} 0\langle X \mid Y\rangle \rightarrow T_{x} 0 X\right)$. Therefore the vector fields 


$$
H\left(\delta x_{k}\right), k \in A, \frac{1}{x_{k}} H_{\xi_{k}}, k \leq \nu, k \in B, H_{\xi_{k}}, k \geq \nu+1, k \in B,
$$

are linearly independent at $x^{0}$. Moreover, the vector fields (2.19.2) commute two by two. Therefore we conclude from Frobenius Theorem that, for a conveniently chosen initial condition along

$$
\left\{p_{j}=0, j \in A, q_{k}=0, k \in B\right\},
$$

$d \xi_{k_{0}\left\langle x^{0}\right\rangle}$ will equal $d p_{k_{0}\left\langle x^{0}\right\rangle}$. Hence we can extend (2.16.1) by $\xi_{k_{0}}$. We can proceed in the same way to extend (2.16.1) by a function $x_{j_{0}}$ for any $j_{0} \notin A$.

We can therefore suppose that $A=[1, n]$ and that there is an integer $l, 1 \leq$ $l \leq \nu$, such that $B=[l+1, n]$.

To finish the proof of the Theorem it is now enough to show that there is a function $f$ such that

$$
\begin{aligned}
\frac{1}{x_{j}}\left\{x_{j}, f\right\} & =\frac{1}{x_{j} x_{l}}\left(\left\{p_{l}, x_{j}\right\}-x_{l} \delta_{j l}\right), \quad 1 \leq j \leq \nu, \\
\left\{x_{j}, f\right\} & =\frac{1}{x_{l}}\left\{p_{l}, x_{j}\right\}, \quad \nu+1 \leq j \leq n, \\
\frac{1}{x_{k}}\left\{\xi_{k}, f\right\} & =\frac{1}{x_{k} x_{l}}\left\{p_{l}, \xi_{k}\right\}, \quad l+1 \leq k \leq n .
\end{aligned}
$$

We notice that, by Remark 2.15, the right hand sides of the equations in (2.19.4) are all holomorphic in a neighbourhood of $x^{0}$. The existence of the function $f$ is guaranteed by the Frobenius theorem. The function

$$
\xi_{l}=p_{l}+x_{l} f
$$

is a residual function along $\left\{x_{l}=0\right\}$ and we can extend (2.16.2) by $\xi_{l}$.

Q.E.D.

Remark 2.20. Let $x_{j}, j \in A, \xi_{k}, k \in B$, be a partial system of logarithmic symplectic local coordinates in an open neighbourhood of a point $x^{0} \in X$. Let $e_{1}, \cdots, e_{n}, f_{1}, \cdots, f_{n}$ be a symplectic basis of $T_{x 0}^{*}\langle X \mid Y\rangle$. Suppose that $\delta x_{j}=$ $e_{j}, d \xi_{k}=f_{k}$, for $j \in A, k \in B$. Then we can choose functions $x_{j}, j \notin A, \xi_{k}, k \notin B$ such that $x_{1}, \cdots, x_{n}, \xi_{1}, \cdots, \xi_{n}$ is a system of logarithmic symplectic local coordinates and $\delta x_{j}=e_{j}, d \xi_{k}=f_{k}$, for $1 \leq j, k \leq n$. Moreover we can arbitrate the values of $x_{j}\left(x^{0}\right), \xi_{k}\left(x^{0}\right)$ for $j \notin A, k \notin B$.

Corollary 2.21. Let $\sigma$ be a logarithmic symplectic form on a complex manifold $X$ with poles along a divisor with normal crossings $Y$. Given $x^{0} \in X$ 
let $\nu$ be the number of irreducible components of $Y$ at $x^{0}$. Then there is a system of local coordinates $\left(x_{1}, \cdots, x_{n}, \xi_{1}, \cdots, \xi_{n}\right)$ on $U$ such that $Y \cap U=\left\{x_{1} \cdots x_{v}\right.$ $=0\}$ and

$$
\left.\sigma\right|_{U}=\sum_{i=1}^{\nu} d \xi_{i} \frac{d x_{i}}{x_{i}}+\sum_{i=\nu+1}^{n} d \xi_{i} d x_{i}
$$

Proof. By Theorem 2.17 and Proposition 2.8 its enough to show that there are holomorphic functions $x_{1}, \cdots, x_{v}$ such that $(2.12 .1)$ holds and

$$
\left\{x_{i}, x_{j}\right\}=0 \quad 1 \leq i, j \leq \nu .
$$

This can be done in the following way: suppose that there are functions $x_{1}, \cdots, x_{y}$ verifying (2.12.1) and an integer $l, 0 \leq l \leq \nu$ such that

$$
\left\{x_{i}, x_{j}\right\}=0 \quad 1 \leq i, j \leq l .
$$

We can show that there is a function $f$ such that

$$
\left\{x_{\dot{z}}, x_{l+1} e^{f}\right\}=0 \quad 1 \leq i \leq l .
$$

Since the method of proof is similar to the one used in Theorem 2.17 we omit it.

Q.E.D.

\section{§3. Homogeneous Logarithmic Symplectic Manifolds}

Let $X$ be a complex manifold. A group action $\alpha: \mathbb{C}^{*} \times X \rightarrow X$ is called a free group action of $\mathbb{C}^{*}$ on $X$ if for each $x \in X$ the isotropy subgroup $\left\{t \in \mathbb{C}^{*}\right.$ : $\alpha(t, x)=x\}$ equals $\{1\}$. A manifold $X$ with a free group action $\alpha$ of $\mathbb{C}^{*}$ is called a conic manifold. We associate to each free group action $\alpha$ of $\mathbb{C}^{*}$ on $X$ a vector field $\rho$, the radial vector field of $\alpha$, in the following way:

$$
\rho f=\left.\frac{\partial}{\partial t} \alpha_{t}^{*} f\right|_{t=1}, \quad f \in \mathcal{O}_{X}
$$

Here $\alpha_{t}(x)=\alpha(t, x)$. We put

$$
\mathcal{O}_{X}(\lambda)=\left\{f \in \mathcal{O}_{X}: \rho f=\lambda f\right\}
$$

for any $\lambda \in \mathbb{C}$ and

$$
\mathcal{O}_{X}^{h}=\bigoplus_{k \in Z} \mathcal{O}_{X}(k)
$$

A section $f$ of $\mathcal{O}_{X}(\lambda)$ is called a homogeneous function of degree $\lambda$. Given conic complex manifolds $\left(X_{1}, \alpha_{1}\right),\left(X_{2}, \alpha_{2}\right)$ a holomorphic map $\varphi: X_{1} \rightarrow X_{2}$ is called homogeneous if it commutes with the actions $\alpha_{1}, \alpha_{2}$, that is, if 


$$
\alpha_{2, t} \varphi=\varphi \alpha_{1, t}, \quad \text { for any } t \in C^{*} .
$$

Definition 3.1. A logarithmic symplectic manifold $(X, \sigma)$ with a free group action $\alpha$ is called a homogeneous symplectic manifold if

$$
\alpha_{t}^{*} \sigma=t \sigma, \quad t \in C^{*} .
$$

If $\left(X_{1}, \sigma_{1}\right),\left(X_{2}, \sigma_{2}\right)$ are homogeneous symplectic manifolds and $\varphi: X_{1} \rightarrow X_{2}$ is a canonical transformation we say that $\varphi$ is a homogeneous canonical transformation if it is homogeneous.

Given a homogeneous logarithmic symplectic manifold $(X, \sigma)$ we call the logarithmic differential form of degree 1

$$
\theta=\imath(\rho) \sigma
$$

the canonical 1-form of $(X, \sigma)$.

We notice that a canonical transformation $\varphi: X_{1} \rightarrow X_{2}$ is a homogeneous canonical transformation if and only if $\varphi^{*} \theta_{2}=\theta_{1}$.

We will now prove a Darboux Theorem in the homogeneous case

Definition 3.2. We say that a partial system of logarithmic symplectic coordinates $x_{j}, j \in A, \xi_{k}, k \in B$, of $(X, \sigma)$ is a partial system of homogeneous logarithmic symplectic coordinates if the functions $x_{j}, j \in A$, are homogeneous of degree 0 and the functions $\xi_{k}, k \in B$, are homogeneous of degree 1 .

Let $(X, \sigma)$ be a homogeneous logarithmic symplectic manifold with poles along a divisor with normal crossings $Y$. Let $U$ be an open set of $X$ and $Y_{0}$ a closed smooth hypersurface contained in $Y \cap U$. A residual function along $Y_{0}$ is called homogeneous if it is homogeneous of degree 1.

If $\xi_{0}, \xi_{0}^{\prime}$ are two homogeneous residual functions along $Y_{0}$ then $\xi_{0}-\xi_{0}^{\prime}$ vanishes along $Y_{0}$. Hence, given $x^{0} \in Y_{0}$ we can define the residual value of $x^{0}$ along $Y_{0}$ as $\xi_{0}\left(x^{0}\right)$.

Theorem 3.3. Let $(X, \mathfrak{S})$ be a homogeneous logarithmic symplectic manifold of dimension $2 n$ with poles along a divisor with normal crossings $Y$. We fix $x^{0} \in$ $X$. Let $\nu$ be the number of irreducible components of $Y_{x^{0}}$. Let

$$
y_{j}, j \in A, \eta_{k}, k \in B,
$$

be a partial system of homogeneous symplectic coordinates on an open neighbourhood $U$ of $x^{0}$ and $b_{k}, 1 \leq k \leq n$, be a family of complex numbers verifying the following conditions: 
(i) The residual value of $x^{0}$ along $Y_{k}$ equals $b_{k}$ for $1 \leq k \leq \nu$.

(ii) $\eta_{k}\left(x^{0}\right)=b_{k}$, for any $k \in B$.

(iii) There is an integer $J$ such that $b_{J} \neq 0$ and $J \notin A \cap[\nu+1, n]$.

Then there is a system of homogeneous logarithmic symplectic coordinates $\left(x_{1}\right.$, $\cdots, x_{n}, \xi_{1}, \cdots, \xi_{n}$ ) on a neigbourhood $V$ of $x^{0}$ such that

$$
\begin{array}{cl}
x_{j} / y_{j} \in \mathcal{O}_{X}^{*}(V) & \text { for } 1 \leq j \leq \nu, \\
x_{j}=y_{j} \quad \text { for } & \nu+1 \leq j \leq n, \\
\xi_{k}=\eta_{k} \quad \text { for } k \in B .
\end{array}
$$

Proof. We may assume that there is an integer $l$ such that $A \cap[1, \nu]=$ $[l+1, \nu]$. Let $A_{0}, B_{0}$ be subsets of $[1, n]$ such that there is a partial system of homogeneous symplectic coordinates

$$
x_{j}, \xi_{k}, \quad j \in A_{0}, k \in B_{0}
$$

verifying (3.3.1). Let

$$
q_{1}, \cdots, q_{n}, p_{1}, \cdots, p_{n}
$$

be a system of logarithmic symplectic coordinates that extends (3.3.2). We will introduce the following assumption:

$$
A_{0}=[1 . \nu] \cup([\nu+1, n] \backslash\{J\}), \quad B_{0}=[l+1, n] .
$$

(3.3.5) We will now show that we can assume $A_{0}=[1, n]$.

We can suppose $J \geq \nu+1$. There are holomorphic functions $a_{k}, 1 \leq k \leq l$, such that relatively to the system of local coordinates (3.3.3),

$$
\rho=\sum_{k=1}^{n} p_{k} \frac{\partial}{\partial p_{k}}+\sum_{k=1}^{l} a_{k} \frac{\partial}{\partial p_{k}}+a \frac{\partial}{\partial q_{J}} .
$$

(3.3.6) Since $d \iota(\rho) \sigma-\sigma=\sum_{k=1}^{l} d a_{k} \delta x_{k}-d a d \xi_{J}$ the functions $a, a_{1}, \cdots, a_{l}$ depend only on $x_{1}, \cdots, x_{l}, \xi_{J}$.

Choose a holomorphic function $f$ depending only on $x_{1}, \cdots, x_{b}, \xi_{J}$ such that

$$
\frac{\partial f}{\partial \xi_{J}}=-\frac{a}{\xi_{J}} .
$$

Then the function $x_{J}=q_{J}+f$ is a homogeneous of degree 0 and we have proved the claim (3.3.5)

Suppose $l \geq 2$. There are holomorphic functions $a_{k}, 1 \leq k \leq l$, such that, relative to the local coordinate system (3.3.3), 


$$
\rho=\sum_{k=1}^{l}\left(p_{k}+a_{k}\right) \frac{\partial}{\partial p_{k}}+\sum_{k=l+1}^{n} \xi_{k} \frac{\partial}{\partial \xi_{k}}
$$

By a reasoning analogous to (3.3.6) the holomorphic functions $a_{k}, 1 \leq k \leq l$ depend only on $x_{1}, \cdots, x_{l}$. Therefore the holomorphic functions $\xi_{k}=p_{k}+a_{k}$ are homogeneous of degree 1 and

$$
\sigma=\sum_{k=1}^{n} d \xi_{k} \delta x_{k} .
$$

(3.3.7) We will now prove (3.3.4) under the hypothesis $J \geq \nu+1$.

Suppose that there is $k_{0}$ such that $k_{0} \geq \nu+1$ and $k_{0} \notin B_{0}$.

Lemma 3.3.8. Let $(E, \sigma)$ be a symplectic vector space of dimension $2 n$. Let $b_{k}, 1 \leq k \leq n$ be a family of complex numbers. Let $\rho_{0}$ be a vector of $E$ and $\varepsilon_{j}, j \in A, \phi_{k}, k \in B, A, B \subset[1, n]$ be a partial symplectic basis of $(E, \sigma)$ verifying the following conditions:

(i) $\sigma_{0}\left(\rho_{0}, \varepsilon_{j}\right)=0, j \in A, \sigma_{0}\left(\rho_{0}, \phi_{k}\right)=b_{k}, k \in B$.

(ii) $\varepsilon_{j}, j \in A, \phi_{k}, k \in B, \rho_{0}$ are linearly independent.

(iii) There is an integer $J$ such that $J \notin A$ and $b_{J} \neq 0$.

Then we can find $\varepsilon_{j}, j \notin A, \phi_{k}, k \notin B$, such that $\varepsilon_{j}, \phi_{k}, 1 \leq j, k \leq n$, is a symplectic basis for $\left(E, \sigma_{0}\right)$ and

$$
\sigma_{0}\left(\rho, \varepsilon_{j}\right)=0, j \in A, \sigma_{0}\left(\rho, \phi_{k}\right)=b_{k}, 1 \leq j, k \leq n .
$$

Proof. cf. (Hörmander [6], Theorem 21.1.9).

Put $E=T_{x} 0\langle X \mid Y\rangle, \sigma_{0}=\sigma_{\left\langle x^{0}\right\rangle}, \rho_{0}=\rho_{\left\langle x^{0}\right\rangle}, \varepsilon_{j}=H\left(e_{j}\right)$ for $j \in A, \phi_{k}=H\left(f_{k}\right)$ for $k \in B$ and $\phi_{k}=H\left(r_{k}\right)$ for $1 \leq k \leq \nu$. By the Lemma 3.3.8 there are vectors $e_{j}$, $j \notin A, f_{k}, k \notin B$ of $T_{x}^{*}\langle X \mid Y\rangle$ such that the vectors $\varepsilon_{j}=H\left(e_{j}\right), \phi_{k}=H\left(f_{k}\right)$ satisfy (3.3.9). We can suppose by Remark 2.20 that the functions (3.3.3) verify the relations

$$
\delta x_{j}\left\langle x^{0}\right\rangle=e_{j} \text { for } j \notin A \text { and } d \xi_{k_{\left\langle x^{0}\right\rangle}}=f_{k}, p_{k}\left(x^{0}\right)=\lambda_{k}, \quad \text { for } k \notin B .
$$

We want to find a function $\xi_{k_{0}}$ such that

$$
\frac{\partial \xi_{k_{0}}}{\partial p_{j}}=\delta_{k_{0} j}, j \in A, \frac{\partial \xi_{k_{0}}}{\partial q_{k}}=0, k \in B, \rho \xi_{k_{0}}=\xi_{k_{0}} .
$$

Therefore we want to find a function $f$, depending only on $p_{j}, j \notin A, q_{k}, k \notin B$, such that

$$
\rho f-f=p_{k_{0}}-\rho p_{k_{0}} .
$$

The equation (3.3.10) is equivalent to the equation 


$$
\rho_{1} f-f=p_{k_{0}}-\rho_{1} p_{k_{0}},
$$

where $\rho_{1}$ is the vector field we obtain after dropping from $\rho$ the coefficients of $\partial / \partial q_{k}, k \in B, \partial / \partial p_{j}, j \in A$. The coefficients of $\rho_{1}$ do not depend on $q_{k}, k \in B, p_{j}$, $j \in A$. Actually take $\rho_{2}=\rho-\Sigma_{j \in A} p_{j}\left(\partial / \partial p_{j}\right)$. Since

$$
\left[H_{f}, \rho\right]=(1-\lambda) H_{f}
$$

for any holomorphic function $f$ of degree $\lambda$ we have

$$
\begin{array}{ll}
{\left[\frac{\partial}{\partial p_{j}}, \rho_{2}\right]=\left[-H_{q_{j}}, \rho\right]-\frac{\partial}{\partial p_{j}}=0,} & j \in A, \\
{\left[\frac{\partial}{\partial q_{k}}, \rho_{2}\right]=\left[-H_{p_{k}}, \rho\right]=0,} & k \in B .
\end{array}
$$

We conclude from (3.3.9) that for any constant $\mu$ there is one and only one solution $f$ of (3.3.10) such that $f-\mu p_{j}$ vanishes along $\left\{p_{J}=b_{J}\right\}$. We can choose $\mu$ in such a way that $d f\left(x^{0}\right)=d p_{k}\left(x^{0}\right)$. We use the function $\xi_{k_{0}}=p_{k_{0}}+f$ to extend the system of partial symplectic coordinates (3.3.2).

We can find in a similar way a function $x_{j_{0}}$ for $j \notin A_{0}$.

(3.3.12) Finally we will prove (3.3.4) under the hypothesis $J \leq \nu$.

Take $A_{1}=A_{0} \backslash\{J\}$. Suppose that there is a $k_{0}$ such that $k_{0} \geq \nu+1$ and $k_{0} \notin$ $B$. We can find, by the procedure described above, a holomorphic function $\xi_{k_{0}}$, homogeneous of degree 1 , such that $\left\{\xi_{k_{0}}, x_{j}\right\}=\delta_{k_{0} j},\left\{\xi_{k_{0}}, \xi_{k}\right\}=0$, for $j \in A$, $k \in B, d \xi_{k_{0}}\left(x^{0}\right)=f_{k_{0}}$, and the function $f=\xi_{k_{0}}-p_{k_{0}}$ depends only on $p_{j}, j \notin A_{1}, q_{k}$, $k \notin B$. Then we want a function $g$, homogeneous of degree 0 , such that

$$
\left\{x_{j}, g\right\}=\left\{\xi_{k}, g\right\}=0, j \in A, k \in B,\left\{\xi_{k_{0}}, x_{J} e^{g}\right\}=0 .
$$

That is, we want a function $g$ depending only on $p_{j}, j \notin A_{1}, q_{k}, k \notin B_{0}$, such that

$$
\left\{\xi_{k_{0}}, g\right\}=-\frac{\partial f}{\partial p_{J}}, \text { and } \rho g=0 .
$$

Since $\left[\rho, H_{\xi_{0}}\right]=0$ the function $g$ exists because of Frobenius Theorem. We can substitute $x_{J}$ by $x_{J} e^{g}$ and $B_{0}$ by $B_{0} \cup\left\{k_{0}\right\}$. We can enlarge the set $A_{0}$ by a similar method.

This ends the proof of Theorem 3.3.

Remark 3.4. We notice that, if there is a $J$ such that $J \notin A$ and $b_{J} \neq 0$ then we can suppose $x_{j}=y_{j}$ for $j \neq J$. If moreover $J \geq \nu+1$ then we can suppose $x_{j}=y_{j}$ for $1 \leq j \leq n$.

A homogeneous logarithmic symplectic manifold is locally isomorphic 
to $\stackrel{\circ}{T}^{*}\langle X \mid Y\rangle$ in the category of homogeneous symplectic manifolds.

Corollary 3.5. Let $\sigma$ be a homogeneous logarithmic symplectic form on a complex manifold $X$ with poles along a divisor nith normal crossings $Y$. Given $x^{0} \in X$ let $\nu$ be the number of irreducible components of $Y$ at $x^{0}$. Then there is a system of local coordinates $\left(x_{1}, \cdots, x_{n}, \xi_{1}, \cdots, \xi_{n}\right)$ on $U$ such that $Y \cap U=\left\{x_{1}\right.$ $\left.\cdots x_{\nu}=0\right\}, x_{1}, \cdots, x_{n}$ are homogeneous of degree $0, \xi_{1}, \cdots, \xi_{n}$ are homogeneous of degree 1 and

$$
\left.\sigma\right|_{U}=\sum_{i=1}^{\nu} d \xi_{i} \frac{d x_{i}}{x_{i}}+\sum_{i=\nu+1}^{n} d \xi_{i} d x_{i}
$$

Proof. It is quite similar to the proof of (2.15). Therefore we omit it

Remark 3.6. If $(X, \sigma)$ is a homogeneous logarithmic symplectic manifold and $x_{j}, 1 \leq j \leq n, \xi_{k}, 1 \leq k \leq n$, is a system of homogeneous logarithmic symplectic coordinates for $\sigma$ on an open set $U$ of $X$ then

$$
\left.\rho\right|_{U}=\sum_{i=1}^{n} \xi_{i} \frac{\partial}{\partial \xi_{i}} \text { and }\left.\theta\right|_{U}=\sum_{i=1}^{n} \xi_{i} \delta x_{i}
$$

Definition 3.7. Given a homogeneous logarithmic symplectic manifold $(X, \sigma)$ with poles along a divisor with normal crossings $Y$ and a smooth hypersurface $Y_{0}$ contained in $Y$ we call residual submanifold of $X$ along $Y_{0}$ to the set of points of $Y_{0}$ of residual value 0 . If $Y$ is smooth we call residual set of $X$ to the residual submanifold of $X$ along $Y$.

Proposition 3.8. Let $X$ be a homogeneous logarithmic symplectic manifold with poles along a smooth divisor $Y$. Let $Z$ be the residual submanifold of $X$. Then:

(i) $Y, Z$ are involutive submanifolds of $X$.

(ii) The manifold $Z$ has a canonical structure of homogeneous symplectic manifold.

Proof. Statement (i) is an immediate consequence of Corollary 3.5.

Let $X_{0}$ be a Poisson manifold. We say that a submanifold $Y_{0}$ of $X_{0}$ is invariant if $\left\{I_{Y_{0}}, \mathcal{O}_{X_{0}}\right\} \subset I_{Y_{0}}$ (cf. Kashiwara Fernandes [10]).

An invariant submanifold of a Poisson manifold has a canonical Poisson structure.

By Corollary 3.5 the set of poles $Y$ is an invariant submanifold of $X$ and the residual submanifold $Z$ is an invariant submanifold of $Y$. Moreover the 
$\mathbb{C}^{*}$ action of $X$ induces a $\mathbb{C}^{*}$-action on $Z$. Given $p^{0} \in Z$ there is a conic neighbourhood $U$ of $p^{0}$ in $X$ and a system of local coordinates $\left(x_{1}, \cdots, x_{n}\right.$, $\xi_{1}, \cdots, \xi_{n}$ ) on $U$ such that $\left.\sigma\right|_{U}=d \xi_{1} d x_{1} / x_{1}+\sum_{i=2}^{n} d \xi_{i} d x_{i}$. We can easily verify that the Poisson structure of $Z \cap U$ is determined by

$$
\sum_{i=2}^{n} d \tilde{\xi}_{i} d \tilde{x}_{i},
$$

where $\tilde{x}_{i}=\left.x_{i}\right|_{z \cap U}, \tilde{\xi}_{i}=\left.\xi_{i}\right|_{z \cap U}$. By Proposition 2.8 (3.8.1) does not depend of the choice of the system of local coordinates $\left(x_{1}, \cdots, x_{n}, \xi_{1}, \cdots, \xi_{n}\right)$ Q.E.D

Example 3.9. Let $X$ be a complex manifold and $Y$ a smooth divisor of $X$. Then the residual submanifold of $\stackrel{\circ}{T}^{*}\langle X \mid Y\rangle$ is isomorphic to $\stackrel{\circ}{T}^{*} Y$.

\section{§4. Logarithmic Contact Mamifolds}

Definition 4.1. Let $X$ be a complex manifold of dimension $2 n+1, n \geq 0$, and $Y$ a divisor with normal crossings of $X$. A local section $\omega$ of $\Omega_{X}^{1}\langle Y\rangle$ is called a logarithmic contact form with poles along $Y$ if $\omega(d \omega)^{n}$ is a local generator of $\Omega_{X}^{2 n+1}\langle Y\rangle$.

We say that a locally free sub $\mathcal{O}_{X}$-module $\mathcal{L}$ of $\Omega_{X}^{1}\langle Y\rangle$ is a logarithmic contact structure on $X$ with poles along $Y$ if it is locally generated by a logarithmic contact form with poles along $Y$. We say that a complex manifold with a logarithmic contact structure with poles along a divisor with normal crossings $Y$ is a logarithmic contact manifold with poles along $Y$. We call $Y$ the set of poles of the logarithmic contact manifold $(X, \mathcal{L})$.

Let $\left(X_{1}, \mathcal{L}_{1}\right),\left(X_{2}, \mathcal{L}_{2}\right)$ be logarithmic contact manifolds. We say that a holomorphic map $\varphi: X_{1} \rightarrow X_{2}$ is a contact transformation if for any local generator of $\mathcal{L}_{2}$ its inverse image by $\varphi$ is a local generator of $\mathcal{L}_{1}$.

Let $Y_{0}$ be a smooth irreducible component of $Y$. We say that a point $x^{0}$ of $Y$ is in the residual set of $X$ along $Y_{0}$ if the residue along $Y_{0}$ of all the sections of $\mathcal{L}$ vanishes at $x^{0}$.

Remark 4.2. (i) Given a logarithmic contact form $\omega$ and a nowhere vanishing holomorphic function $\varphi, \varphi \omega$ is a logarithmic contact form.

(ii) We say that two logarithmic contact forms $\omega_{1}, \omega_{2}$, are equivalent if there is a nowhere vanishing holomorphic function $\varphi$ such that $\omega_{2}=\varphi \omega_{1}$.

(iii) We notice that it is equivalent to give a structure of logarithmic contact manifold along $Y$ and to give an open covering $\left(U_{i}\right)$ of $X$ and logarithmic contact forms $\omega_{i} \in \Gamma\left(U_{i}, \Omega_{X}^{1}\langle Y\rangle\right)$ with poles along $U_{i} \cap Y$ and verifying the condition 
" $\omega_{i}$ is equivalent to $\omega_{j}$ on $U_{i} \cap U_{j}$ ".

Proposition 4.3. There is an equivalence of categories between the category of logarithmic contact manifolds and the category of homogeneous logarithmic symplectic manifolds.

Proof. Let $(X, \mathcal{L})$ be a logarithmic contact manifold along a divisor with normal crossings $Y$. We put

$$
\hat{X}=\operatorname{Specan}\left(\oplus_{k \in Z^{2}} \mathcal{L}^{\otimes(-k)}\right) .
$$

We will denote by $r$ the canonical projection $\hat{X} \rightarrow X$. The complex manifold $\hat{X}$ with the projection $r$ is the $\boldsymbol{C}^{*}$-bundle we obtain after removing the zero section of the line bundle associated to $\mathcal{L}$. Moreover $\hat{X}$ has a canonical structure of homogeneous symplectic manifold. Actually let $\omega$ be a local generator of $\mathcal{L}$. Locally

$$
\hat{X}=\operatorname{Specan}\left(\mathcal{O}_{X}\left[\omega, \omega^{\otimes-1}\right]\right)
$$

Let $\eta$ be the image of $\omega^{\otimes-1}$ by the canonical morphism $\gamma^{-1} \mathcal{O}_{X}\left[\omega, \omega^{\otimes-1}\right] \rightarrow \mathcal{O}_{\hat{X}}$. The logarithmic differential form $\eta r^{*} \omega$ does not depend on the choice of $\omega$ and $d\left(\eta r^{*} \omega\right)$ is a logarithmic symplectic form with poles along $r^{-1}(Y)$.

(4.3.2) The logarithmic differential form $\eta r^{*} \omega$ is the canonical 1-form of the logarithmic symplectic manifold $\hat{X}$.

Choose a system of local coordinates $x_{i}, 1 \leq i \leq 2 n+1$, in the open set $U$ of $X$ where $\omega$ is defined. Then $\eta, r^{*} x_{i}, 1 \leq i \leq 2 n+1$, is a system of local coordinates of $\hat{X}$ on $\gamma^{-1} U$ and relatively to this system of coordinates the radial vector fileld $\rho$ of $\hat{X}$ is given by $\eta \partial / \partial \eta$. Now the statement (4.3.2) follows from the equality

$$
i\left(\eta \frac{\partial}{\partial \eta}\right)\left(\eta d r^{*} \omega+d \eta \cdot r^{*} \omega\right)=\eta r^{*} \omega
$$

(4.3.3) We notice that if $Y_{0}$ is a smooth hypersurface contained in $Y$ and $Z_{0}$ is the residual set of $X$ along $Y_{0}$ then the set of poles of $\hat{X}$ equals $\gamma^{-1} Y$ and the residual set of $\hat{X}$ along $Y_{0}$ equals $\gamma^{-1} Z_{0}$.

Let now $X$ be a homogeneous logarithmic symplectic manifold. Let $\theta$ be the canonical 1-form of $X$ and let $Y$ be the set poles of $X$. Let $X_{*}$ be the quotient of $X$ by its $C^{*}$ action. Then $X_{*}$ is a complex manifold and the canonical epimorphism $r: X \rightarrow X_{*}$ is a $\boldsymbol{C}^{*}$-bundle. Put $Y_{*}=r(Y)$. Let $\mathcal{L}_{*}$ be the sub $\mathcal{O}_{X_{*}}$-module of $\Omega_{X_{*}}^{1}\left\langle Y_{*}\right\rangle$ generated by the logarithmic differential forms $s^{*} \theta$, where $s$ is a holomorphic section of $r$. Then $\mathcal{L}_{*}$ is a structure of logarithmic 
contact manifold with poles along $Y_{*}$.

Q.E.D.

Remark 4.4. Let $X$ be a logarithmic contact manifold and $\hat{X}$ the associated homogeneous logarithmic symplectic manifold. Let $r: \hat{X} \rightarrow X$ be the canonical projection. From now on we will often identify $X$ with $\hat{X}$. We will also identify a sheaf $\mathcal{E}$ on $\hat{X}$ that is constant along the fibers of $r$ with the sheaf $r_{*} \mathcal{E}$ on $X$.

Let $\mathbb{P}^{*}\langle X \mid Y\rangle$ be the projective bundle associated to $T^{*}\langle X \mid Y\rangle$. We call $\mathbb{P}^{*}\langle X \mid Y\rangle$ the projective logarithmic cotangent bundle of $X$ with poles along $Y$.

The projective bundle $\mathbb{P}^{*}\langle X \mid Y\rangle$ has a canonical structure of logarithmic contact manifold. Moerover the associated homogeneous logarithmic symplectic manifold equals $\stackrel{\circ}{T}^{*}\langle X \mid Y\rangle$.

A logarithmic contact manifold of dimension $2 n$ is locally isomorphic to $\mathbb{P}^{*}\left\langle\mathbb{C}^{n} /\left\{x_{1} \cdots x_{2}=0\right\}\right\rangle$, for some integer $\nu$.

Theorem 4.5. Let $X$ be a complex manifold of dimension $2 n+1$.

(i) Let $\omega$ be a logarithmic contact form of $X$. Given a point $x^{0}$ in the domain of $\omega$ there are holomorphic functions $x_{1}, \cdots, x_{n+1}, \zeta_{1}, \cdots, \zeta_{n+1}$ defined in an open neighbourhood $U$ of $X$ such that

$$
\left.\omega\right|_{U}=\sum_{i=1}^{n+1} \zeta_{i} \delta x_{i}
$$

Moreover there is an $i$ such that $\zeta_{i}\left(x^{0}\right) \neq 0$ and for any $i_{0}$ such that $\zeta_{i_{0}}\left(x^{0}\right) \neq 0$ the functions

$$
x_{i}, 1 \leq i \leq n+1, \frac{\zeta_{i}}{\zeta_{i_{0}}}, 1 \leq i \leq n+1, i \neq i_{0}
$$

are a local system of coordinates for $X$ on $U$.

(ii) Let $\mathcal{L}$ be a logarithmic contact structure on $X$ with poles along a divisor with normal crossings $Y$. Given a point $x^{0}$ of $X$, suppose that $Y_{x_{0}}$ has irreducible components $Y_{1}, \cdots, Y_{\nu}$ and that the residual values of $x^{0}$ along $Y_{i}$ vanish for $1 \leq i \leq \nu$. Then there is a system of coordinates $\left(x_{1}, \cdots, x_{n+1}, p_{1}, \cdots, p_{n}\right)$ in $a$ neighbourhood $U$ of $x^{0}$ such that the logarithmic differential form

$$
d x_{n+1}-\sum_{i=1}^{\nu} p_{i} \frac{d x_{i}}{x_{i}}-\sum_{i=v+1}^{n} p_{i} d x_{i}
$$

is a local generator of $\mathcal{L}$ and $Y_{i} \cap U=\left\{x_{i}=0\right\}$, for $1 \leq i \leq \nu$. 
Proof. Let $X^{\prime}$ be the domain of $\omega$. Let $Y$ be the set of poles of $\omega$. Let $\nu$ be the number of irreducible components of $Y$ at $x^{0}$. Put $\mathcal{L}=\mathcal{O}_{X^{\prime}} \omega$. Choose $y^{0} \in \hat{X}^{\prime}$ such that $r\left(y^{0}\right)=x^{0}$. We can find a system of homogeneous symplectic coordinates $x_{j}, 1 \leq j \leq n+1, \xi_{k}, 1 \leq k \leq n+1$, such that

$$
\eta r^{*} \omega=\sum_{i} \xi_{i} \delta x_{i}
$$

and $\gamma^{-1}(Y) \cap V=\left\{x_{1} \cdots x_{v}=0\right\}, x_{i}\left(y^{0}\right)=0$ for $\nu+1 \leq i \leq n$. We can suppose $V=$ $r^{-1}(U)$ for some open neighbourhood $U$ of $x^{0}$. The functions $x_{i}, \xi_{i} / \eta, 1 \leq i \leq$ $n+1$ are homogeneous of degree 0 and therefore determine holomorphic functions on $U$ that we will denote respectively by $x_{i}, \zeta_{i}$. They obviously satisfy (4.5.1).

Suppose now that $\xi_{i}\left(y^{0}\right)=0$ for $1 \leq i \leq n+1$. Then the set $\left\{y^{0}\right\}$ would be invariant by the action of $\alpha$ and $\alpha$ wouldn't be a free group action. Therefore there is an $i$ such that $\zeta_{i}\left(x^{0}\right) \neq 0$.

Finally if $\zeta_{i_{0}}\left(x^{0}\right) \neq 0$ then $\xi_{i_{0}}\left(y^{0}\right) \neq 0$ and

$$
\delta x_{i_{0}}+\sum_{i \neq i_{0}} \frac{\xi_{i}}{\xi_{i_{0}}} \delta x_{i}
$$

determines a logarithmic contact form on $U$. Since there is a nonvanishing constant $C$ such thai

$$
\omega_{0}\left(d \omega_{0}\right)^{n} \equiv C \delta x_{1} \cdots \delta x_{n+1} \wedge_{i \neq i_{0}} d \frac{\zeta_{i}}{\zeta_{i_{0}}}\left(\bmod W_{\nu-1}\left(\Omega_{X}^{2 n+1}\langle Y\rangle\right)\right)
$$

the differential form $d x_{1} \cdots d x_{n+1} \wedge_{i \neq i_{0}} d \zeta_{i} / \zeta_{i_{0}}$ does not vanish at $x^{0}$. This proves (i). We can suppose $i_{0}=n+1$. If we put $p_{i}=-\zeta_{i} / \zeta_{n+1}$, for $1 \leq i \leq n$ then $\omega_{0}=$ (4.5.2).

Q.E.D.

\section{Chapter II. Quantized Logarithmic Contact Manifolds}

Let $X$ be a complex manifold and $Y$ a divisor with normal crossings of $X$. In $\S 5$ we will build a sheaf $\mathcal{E}_{\langle X / Y\rangle}$ on the vector bundle $T^{*}\langle X \mid Y\rangle$. This sheaf is a natural generalization of the sheaf $\mathcal{E}_{X}$ of microdifferential operators on $T^{*} X$. It is a "microlocalization" of the sheaf $\mathscr{D}_{X}\langle Y\rangle$ of the differential operators tangent to $Y$ in the same sense $\mathcal{E}_{X}$ is a "microlocalization" of $\mathscr{D}_{X}$.

Theorem 5.10 and its Corollaries will be systematically used through all the paper. Roughly speaking they allow us to extend results on "noncommutative polynomials" to "non commutative power series".

In $\S 8$ we introduce the notions of quantized logarithmic contact manifold 
and self dual quantized logarithmic contact manifold. Roughly speaking a quantized logarithmic contact manifold is a ringed space $(M, \mathcal{E})$ where $M$ is a logarithmic contact manifold and moreover $(M, \mathcal{E})$ is locally isomorphic to the ringed space $\left(\mathbb{P}^{*}\langle X \mid Y\rangle, \mathcal{E}_{\langle X / Y\rangle}\right)$. We call the sheaf $\mathcal{E}$ a quantization of the contact manifold $M$. A self dual quantized logarithmic contact manifold is a quantized logarithmic contact manifold with a globally defined adjoint morphism similar to the adjoint morphism locally defined in $\mathcal{E}_{X}$.

We introduce a globally defined notion of subprincipal symbol of a sectoin of a self dual quantized logarithmic contact manifold and use it to prove the global existence of the both side ideals that where locally studied in $\$ 6$. This ideals are deeply related with the set of poles and the residual submanifold of the underlying logarithmic contact manifold.

\section{§5. The Sheat of Logarithmic Microdifferential Operators}

Let $X$ be a complex manifold and $\mathcal{L}$ a Lie algebra of derivations of $\mathcal{O}_{X}$ that is a locally free $\mathcal{O}_{X}$-module. Let $\mathscr{D}_{\mathcal{L}}$ be the sub $\mathcal{O}_{X}$-algebra of the sheaf of differential operators $\mathscr{D}_{X}$ generated by $\mathcal{L}$. We endow $\mathscr{D}_{\mathcal{L}}$ with the filtration induced by the canonical filtration of $\mathscr{D}_{X}$.

Proposition 5.1. The vector bundle $X_{\mathcal{L}}=\operatorname{Specan}\left(\mathrm{gr} \mathscr{D}_{\mathcal{L}}\right)$ has a canonical structure of Poisson manifold.

Proof. Let $\pi$ be the canonical projection from $X_{\mathcal{L}}$ onto $X$. The Lie bracket of $\mathscr{D}_{\mathcal{L}}$ induces a structure of Poisson algebra in $\operatorname{gr} \mathscr{D}_{\mathcal{L}}$. Moreover the sheaves $\mathcal{O}_{\left[X_{\mathcal{L}}\right]}:=\pi^{-1} \pi_{*} \mathcal{O}_{X_{\mathcal{L}}^{h}}$ and $\pi^{-1} \operatorname{gr} \mathscr{D}_{\mathcal{L}}$ are isomorphic. We obtain in this way canonical morphisms of sheaves

$$
\begin{gathered}
\sigma \frac{\mathcal{L}}{m}: \pi^{-1} \mathscr{D}_{\mathcal{L}}(m) \rightarrow \mathcal{O}_{X \mathcal{L}^{(m)},}, \\
\sigma \mathcal{L}_{:} \pi^{-1} \mathscr{D}_{\mathcal{L}} \rightarrow \mathcal{O}_{X_{\mathcal{L}}}^{h} .
\end{gathered}
$$

Let $U$ be an open set of $X,\left(x_{1}, \cdots, x_{n}\right)$ a system of local coordinates of $X$ on $U$ and $u_{1}, \cdots, u_{k}$ a basis of $\left.\mathcal{L}\right|_{U}$. Then $\left(x_{1}, \cdots, x_{n}, \xi_{1}, \cdots, \xi_{k}\right)$ is a system of local coordinates of $X_{\mathcal{L}}$ on $\pi^{-1}(U)$, where $\xi_{i}=\sigma\left(u_{\dot{j}}\right)$ for $1 \leq i \leq k$.

The Poisson structure of $\mathcal{O}_{\left[X_{\mathcal{L}}\right]}$ is determined by

$$
\{f, g\}=0, \quad\left\{\xi_{i}, f\right\}=u_{i} f, \quad\left\{\xi_{i}, \xi_{j}\right\}=\sigma\left(\left[u_{i}, u_{j}\right]\right),
$$

for $f, g$ local sections of $\pi^{-1} \mathcal{O}_{X}, 1 \leq i, j \leq n$. Since for any $x^{0} \in X_{\mathcal{L}}$ there is a unique extension of the Poisson structure of $\mathcal{O}_{\left[X_{\mathcal{L}}\right], x^{0}}$ to $\mathcal{O}_{X_{\mathcal{L}}, x^{0}}$ then it is 
enough to show that it is possible to extend the Poisson structure of $\mathcal{O}_{\left[X_{\mathcal{L}}\right]}$ to $\mathcal{O}_{X_{\mathcal{L}}}$ locally. If $u_{i}=\sum_{j} a_{i j} \partial_{x_{j}}$ an $\left[u_{i}, u_{j}\right]=\sum_{l} b_{i j}^{l} u_{l}$ we define

$$
\{f, g\}=\sum_{i, j}\left(a_{i j}\left(\frac{\partial f}{\partial \xi_{i}} \frac{\partial g}{\partial x_{j}}-\frac{\partial f}{\partial x_{i}} \frac{\partial g}{\partial \xi_{j}}\right)+\sum_{l} b_{i j}^{l}\left(\frac{\partial f}{\partial \xi_{i}} \frac{\partial g}{\partial \xi_{j}}-\frac{\partial f}{\partial \xi_{j}} \frac{\partial g}{\partial \xi_{i}}\right) \xi_{l}\right)
$$

for any section $f, g$ of $\mathcal{O}_{X_{\mathcal{L}}}$.

Q.E.D.

Definition 5.2. Let $Y$ be a divisor with normal crossings of $X$. We denote by $\mathscr{D}_{X}\langle Y\rangle$ the ring $\mathscr{D}_{\Theta_{X}}\langle Y\rangle$ and call it the ring of differential operators of $X$ tangent to $Y$. We will denote by $\sigma_{Y}$ the principal symbol morphism $\sigma^{\Theta_{X}}\langle Y\rangle$ introduced in the proof of Proposition 5.1.

Proposition 5.3. The vector bundles $X_{\Theta_{X}}\langle Y\rangle$ and $T^{*}\langle X \mid Y\rangle$ are isomorphic as vector bundles and as Poisson manifolds.

Proof. Since gr $\mathscr{D}_{X}\langle Y\rangle$ is isomorphic to $\mathrm{S}\left(\Theta_{X}\langle Y\rangle\right)$, the symmetric algebra of $\left(\Theta_{X}\langle Y\rangle\right)$, then Specan $\left(\operatorname{gr} \mathscr{D}_{X}\langle Y\rangle\right)$ equals the dual of the vector bundle with sheaf of sections $\Theta_{X}\langle Y\rangle$. Given a system of local coordinates $\left(x_{1}, \cdots, x_{n}\right)$ on an open set $U$ of $X$ subordinated to $Y \cap U$ let $(x, \xi)$ be the associated system of canonical coordinates in $T^{*}\langle X \mid Y\rangle$. For $1 \leq i \leq n$ put $\eta_{i}=\sigma_{Y}\left(\delta_{x_{i}}\right)$, where $\delta_{x_{i}}$ is the vector field introduced in section 1.1. The functions $\left(x_{1}, \cdots, x_{n}, \eta_{1}, \cdots, \eta_{n}\right)$ define a system of homogeneous logarithmic symplectic coordinates outside the zero section. Therefore $\left\{\xi_{i}-\eta_{i}, x_{j}\right\}$ vanishes for $1 \leq i, j \leq n$. Hence $\xi_{i}-\eta_{i}$ depends only on $x_{1}, \cdots, x_{n}$. We conclude that the functions $\xi_{i}-\eta_{i}$ are homogeneous of degree 0 and 1 . Therefore

$$
\sigma_{Y}\left(\delta_{x_{i}}\right)=\xi_{i}, \quad 1 \leq i \leq n . \quad \text { Q.E.D. }
$$

Definition 5.4. Let $U$ be an open set of $X$ and let $\left(x_{1}, \cdots, x_{n}\right)$ be a system of local coordinates subordinated to the divisor with normal crossings $Y \cap U$. Given a section $P$ of $\mathscr{D}_{X}\langle Y\rangle$ we define the total symbol of $P$ as the element $\left(P_{j}\right)$ of $\mathcal{O}_{\left[T^{*}\langle X / Y\rangle\right]}$ determined by

$$
e^{-\langle x, \xi\rangle_{\nu}} P e^{\langle x, \xi\rangle_{\nu}}
$$

where $\langle x, \xi\rangle_{\nu}=\sum_{i=1}^{\nu} \xi_{i} \log x_{i}+\sum_{i=v+1}^{n} x_{i} \xi_{i}$.

Proposition 5.5. (i) Given two sections $P, Q$ of $\mathscr{D}_{X}\langle Y\rangle$

$$
\begin{gathered}
(P+Q)_{l}=P_{l}+Q_{l} \\
(P Q)_{l}=\sum_{\substack{l=j+k-|\alpha| \\
\alpha \in N^{n}}} \frac{1}{\alpha !}\left(\partial_{\xi}^{\alpha} P_{j}\right)\left(\delta_{x}^{\alpha} Q_{k}\right) .
\end{gathered}
$$


(ii) If $\left(\tilde{x}_{1}, \cdots, \tilde{x}_{n}\right)$ is another system of local coordinates of $X_{n}$ on $U$ such that $Y \cap U=\left\{\tilde{x}_{1}, \cdots, \tilde{x}_{\nu}=0\right\}$ and $\tilde{x}_{i} / x_{i}$ is holomorphic for $1 \leq i \leq \nu$ then the associated systems of canonical coordinates are related by

$$
\xi_{k}=\frac{\delta\langle\tilde{x}, \tilde{\xi}\rangle_{v}}{\delta x_{k}}, \quad 1 \leq k \leq n
$$

Moreover, for any $l \in \mathbb{Z}$,

$$
\widetilde{P}_{l}(\tilde{x}, \tilde{\xi})=\sum_{\substack{\sigma \\ \alpha_{1}, \cdots, \alpha_{\sigma}}} \frac{1}{\sigma ! \alpha_{1} ! \cdots \alpha_{\sigma} !}\left\langle\tilde{\xi}, \delta_{x}^{\alpha_{1}} \tilde{x}\right\rangle_{\nu} \cdots\left\langle\tilde{\xi}, \delta_{x}^{\alpha_{\sigma}} \tilde{x}\right\rangle_{\nu} \partial_{\xi}^{\alpha_{1}+\cdots+\alpha_{\sigma}} P_{k}(x, \xi) .
$$

Here the indexes run over $k \in \mathbb{Z}, \sigma \in \mathbb{N}, \alpha_{1}, \cdots, \alpha_{\sigma} \in \mathbb{N}^{n}$, such that $\left|\alpha_{1}\right|, \cdots,\left|\alpha_{\sigma}\right|$ $\geq 2$ and $l=k+\sigma-\sum_{i=1}^{\sigma}\left|\alpha_{i}\right|$. For $\beta \in N^{n}\left\langle\tilde{\xi}, \delta_{x}^{\beta} \tilde{x}\right\rangle_{\nu}$ denotes

$$
\sum_{j=1}^{\nu} \tilde{\xi}_{j} \delta_{x}^{\beta} \log \tilde{x}_{j}+\sum_{j=\nu+1}^{n} \tilde{\xi}_{j} \delta_{x}^{\beta} \tilde{x}_{j}
$$

Proof. The proof of this Proposition is the obvious generalization of the proof in the case $\nu=0$. By (5.5.1) its enough to prove (5.5.2) and (5.5.3) when $P=\xi^{\alpha}$. This can be accomplished by induction in $|\alpha|$. The induction step of (5.5.3) uses (5.5.2).

Q.E.D.

Definition 5.6. Let $X_{n}$ be a copy of $\mathbb{C}^{n}$ with coordinates $\left(x_{1}, \cdots, x_{n}\right)$. Let $\nu$ be an integer smaller or equal to $n$ and $Y_{\nu}$ the divisor with normal crossings $\left\{x_{1} \cdots x_{\nu}=0\right\}$ of $X_{n}$. Let $U$ be an open set of $T^{*}\left\langle X_{n} / Y_{\nu}\right\rangle$. We denote by

$$
\hat{\varepsilon}_{\left\langle X_{n} / Y_{\nu}\right\rangle}(m)(U)
$$

the space of formal series $\sum_{j \leq m} P_{j}$ where $P_{j}$ is a section of $\mathcal{O}_{T^{*}\left\langle X_{n} / Y_{\nu}\right\rangle(j)}$ on $U$. The correspondence

$$
U \mapsto \hat{\varepsilon}_{\left\langle X_{n} / Y_{y}\right\rangle}(m)(U)
$$

defines a sheaf of $\boldsymbol{C}$-modules denoted $\hat{\varepsilon}_{\left\langle x_{n} / Y_{\nu}\right\rangle}{ }^{(m)}$. We put

$$
\hat{\varepsilon}_{\left\langle X_{n} / Y_{\nu}\right\rangle}=U_{m} \hat{\varepsilon}_{\left\langle X_{n} / Y_{\nu}\right\rangle}{ }^{(m)} \text {. }
$$

Given sections $P=\sum P_{j}, Q=\sum Q_{k}$ of $\hat{\varepsilon}_{\left\langle X_{n} / Y_{\nu}\right\rangle}$ defined in an open set $U$ of $T^{*}\left\langle X_{n} / Y_{y}\right\rangle$ we define the sum and product of $P$ and $Q$ respectively by the formulas (5.5.1) and (5.5.2). We say that a section $\sum P_{j}$ of $\hat{\mathcal{E}}_{\left\langle X_{n} / Y_{\nu}\right\rangle}(U)$ is a $\log$ arithmic microdifferential operator if for any compact set $K$ of $U$ there is a constant $C$ such that

$$
\sup _{K}\left|P_{-j}\right| \leq C^{j} j !, \quad j \geq 0
$$


We will denote by $\mathcal{E}_{\left\langle X_{n} / Y_{\nu}\right\rangle}$ the subsheaf of $\hat{\mathcal{E}}_{\left\langle X_{n} / Y_{\nu}\right\rangle}$ whose sections are logarithmic microdifferential operators. We will consider $\mathcal{E}_{\left\langle X_{n} / Y_{\nu}\right\rangle}$ endowed with the filtration induced by $\hat{\mathcal{E}}_{\left\langle X_{n} / Y_{\nu}\right\rangle}$.

We will denote the section $\left(\xi_{i}\right)$ by $\delta_{x_{i}}$ for $1 \leq i \leq n$. If $\nu+1 \leq i \leq n$ then we will usually denote $\left(\xi_{i}\right)$ by $\partial_{x_{i}}$ instead of $\delta_{x_{i}}$. We introduce the following convention. If in a statement we denote a $\left(\xi_{i}\right)$ by $\partial_{x_{i}}$ we will do it in that statement whenever possible.

Let $X_{n}$ be a copy of $C^{n}$ with coordinates $\left(x_{1}, \cdots, x_{n}\right)$. Suppose $\mu \leq \nu$ and put $Y_{\mu_{\nu}}=\left\{x_{\mu_{+1}} \cdots x_{\nu}=0\right\}$. Let $\iota$ denote the canonical isomorphism

$$
\left.\left.T^{*}\left\langle X_{n} / Y_{\mu}\right\rangle\right|_{X_{n} \backslash Y_{\mu \nu}} \underset{\rightarrow}{\rightarrow} T^{*}\left\langle X_{n} / Y_{\nu}\right\rangle\right|_{X_{n} \backslash Y_{\mu \nu}}
$$

If we consider in $T^{*}\left\langle X_{n} / Y_{\nu}\right\rangle\left[T^{*}\left\langle X_{n} / Y_{\mu}\right\rangle\right]$ the system of canonical coordinates $(x, \xi)[(x, \tilde{\xi})]$ associated to $(x)$ then

$$
\begin{array}{ll}
\iota^{*} \xi_{i}=\tilde{\xi}_{i}, & \text { if } 1 \leq i \leq \nu \text { or } \nu+1 \leq i \leq n, \\
\iota^{*} \xi_{i}=x_{i} \tilde{\xi}_{i}, & \text { if } \mu+1 \leq i \leq \nu .
\end{array}
$$

Proposition 5.7. (i) The sheaves $\mathcal{E}_{\left\langle x_{n} / Y_{\nu}\right\rangle}$ and $\hat{\mathcal{E}}_{\left\langle x_{n} / Y_{\nu}\right\rangle}$ are associative $\boldsymbol{C}$ Algebras.

(ii) Let $X_{n}\left[\tilde{X}_{n}\right]$ be a copy of $C^{n}$ with coordinates $\left(x_{1}, \cdots, x_{n}\right)\left[\left(y_{1}, \cdots, y_{n}\right)\right]$. Let $\tilde{X}=\left(\tilde{x}_{1}, \cdots, \tilde{x}_{n}\right)$ be a biholomorphic map from an open set $U$ of $X_{n}$ into an open set $V$ of $\tilde{X}_{n}$. Put $Y_{\nu}=\left\{x_{1} \cdots x_{\nu}=0\right\}, \hat{Y}_{\nu}=\left\{y_{1} \cdots y_{\nu}=0\right\}$. If $\tilde{x}^{-1}\left(\hat{Y}_{\nu}\right)=Y_{\nu} \cap U$ then formula (5.3.3) defines isomorphisms of filtered $\boldsymbol{C}$-Algebras

$$
\begin{aligned}
& \left.\left.\mathcal{E}_{\left\langle X_{n} / Y_{\nu}\right\rangle}\right|_{U} \underset{\sim}{\rightarrow} \mathcal{E}_{\left\langle\tilde{X}_{n} / \tilde{Y}_{\nu}\right\rangle}\right|_{V} \\
& \left.\hat{\varepsilon}_{\left\langle X_{n} / Y_{\nu}\right\rangle}\right|_{U} \underset{\left.\hat{\varepsilon}_{\left\langle\tilde{X}_{n} / \tilde{Y}_{\nu}\right\rangle}\right|_{V}}{ }
\end{aligned}
$$

(iii) There are canonical isomorphisms

$$
\begin{aligned}
& \left.\left.\mathcal{E}_{\left\langle X_{n} / Y_{\nu}\right\rangle}\right|_{X_{n} \backslash Y_{\mu \nu}} \underset{\sim}{\sim} \mathcal{E}_{\left\langle X_{n} / Y_{\mu}\right\rangle}\right|_{X_{n} \backslash Y_{\mu \nu}} \\
& \left.\hat{\mathcal{E}}_{\left\langle X_{n} / Y_{\nu}\right\rangle}\right|_{X_{n} \backslash Y_{\mu \nu}} \underset{\left.\mathcal{E}_{\left\langle X_{n} / Y_{\mu}\right\rangle}\right|_{X_{n} \backslash Y_{\mu \nu}}}{ }
\end{aligned}
$$

The isomorphisms (5.7.2) are explicitly given by

$$
\widetilde{P}_{l}(x, \tilde{\xi})=\sum_{\substack{\sigma \\ \alpha_{1}, \cdots, \alpha_{\sigma}}} \frac{1}{\sigma ! \alpha_{1} ! \cdots \alpha_{\sigma} !}\left(\sum_{i=\mu+1}^{\nu} \xi_{i}\right)^{\sigma} \partial_{\xi}^{\alpha_{1}+\cdots+\alpha_{\sigma}} P_{k}(x, \xi) .
$$

Here the indexes run over $k \in Z, \alpha_{1}, \cdots, \alpha_{\sigma} \in\{0\}^{\mu} \times \boldsymbol{N}^{\nu-\mu} \times\{0\}^{n-\nu}$ such that $\left|\alpha_{1}\right|, \cdots,\left|\alpha_{\sigma}\right| \geq 2$ and $l=k+\sigma-\sum_{i=1}^{\sigma}\left|\alpha_{i}\right|$. In particular 


$$
\begin{aligned}
& \delta_{x_{i}} \mapsto \delta_{x_{i}}, \quad \text { for } 1 \leq i \leq \mu \text {, } \\
& \delta_{x_{i}} \mapsto x_{i} \partial_{x_{i}}, \quad \text { for } \mu+1 \leq i \leq \nu \text {, } \\
& \partial_{x_{i}} \mapsto \partial_{x_{i}}, \quad \text { for } \nu+1 \leq i \leq n \text {. }
\end{aligned}
$$

(iv) The correspondence " $P \mapsto$ total symbol of $P$ " defines an immersion of $\pi^{-1} \mathscr{D}_{X_{n}}\left\langle Y_{\nu}\right\rangle$ into $\mathcal{E}_{\left\langle X_{n} / Y_{\nu}\right\rangle}$.

(v) The restriction of $\mathcal{E}_{\left\langle X_{n} / Y_{\nu}\right\rangle}\left[\hat{\mathcal{E}}_{\left\langle X_{n} / Y_{\nu}\right\rangle}\right]$ to the zero section of $T^{*}\left\langle X_{n} / Y_{v}\right\rangle$ equals $\mathscr{D}_{X_{n}}\left\langle Y_{\nu}\right\rangle$.

Lemma 5.7.1. Let $X_{n}^{\prime}$ be the open set $\left\{0<\operatorname{Im~} z_{\dot{\xi}}<2 \pi, \mu+1 \leq i \leq \nu\right\}$ of $a$ copy $Z_{n}$ of $\mathbb{C}^{n}$ with coordinates $\left(z_{1}, \cdots, z_{n}\right)$. Let $(z, \zeta)$ be the system of coordinates of $T^{*}\left\langle Z_{n} / Y_{\mu}\right\rangle$ associated to $(z)$. Let $\chi: X_{n}^{\prime} \rightarrow X_{n} \backslash Y_{\mu \nu}$ be the biholomorphic map defined by:

$$
\begin{array}{ll}
\chi^{*} x_{i}=e^{z_{i}}, & \text { if } \mu+1 \leq i \leq \nu, \\
\chi^{*} x_{\dot{i}}=z_{i}, & \text { if } 1 \leq i \leq \mu \text { or } \nu+1 \leq i \leq n .
\end{array}
$$

Let $\tilde{\chi}: T^{*}\left\langle X_{n} \mid Y_{\nu}\right\rangle\left|{ }_{X_{n} \backslash Y_{\mu \nu}} \rightarrow T^{*}\left\langle Z_{n} \mid Y_{\nu}\right\rangle\right|_{X_{n}^{\prime}}$ be the biholomorphic map induced by $\chi$. Then the following morphism of sheaves is an isomorphism of filtered $\mathbb{C}$ Algebras

$$
\begin{gathered}
\left.\left.\hat{\mathcal{E}}_{\left\langle Z_{n} / Y_{\mu}\right\rangle}\right|_{X_{n}^{\prime}} \stackrel{\sim}{\rightarrow} \hat{\mathcal{E}}_{X n / Y,}\right|_{X_{n} \backslash Y \mu \nu} \\
\sum_{j} P_{j} \mapsto \sum_{j} P_{j} \circ \tilde{\chi}
\end{gathered}
$$

Moreover $\Sigma_{j} P_{j}$ is a logarithmic microdifferential operator iff $\Sigma_{j} P_{j} \circ \tilde{\chi}$ is a logarithmic microdifferential operator.

Proof. It is an immediate consequence of the following facts. For $1 \leq i \leq n$

$$
\chi_{*}\left(\left.\partial_{z_{i}}\right|_{X_{n}^{\prime}}\right)=\delta_{x_{i}}, \quad \tilde{\chi}^{*} \zeta_{i}=\xi_{i}, \quad \tilde{\chi}\left(\left.\partial_{\xi_{i}}\right|_{X_{n}^{\prime}}\right)=\partial_{\xi_{i} \cdot} \quad \text { Q.E.D. }
$$

Proof of Proposition 5.7. (i) By Lemma 5.7.1 if $P, Q, R \in \hat{\mathcal{E}}_{\left\langle X_{n} / Y_{\nu}\right\rangle}(U)$ then $(P Q) R=P(Q R)$ in an open dense subset of $U$. Therefore $\hat{\mathcal{E}}_{\left\langle X_{n} / Y_{v}\right\rangle}$ is an associative Algebra. Also by Lemma 5.7.1 if $P, Q$ are logarithmic microdifferential operators then $P Q$ is a logarithmic microdifferential operator outside of a divisor with normal crossings of $U$. By the Cauchy estimates $P Q$ is a logarithmic microdifferential operator on $U$.

(ii) By the remarks made in the proof of Lemma 5.7.1 we can deduce (ii) from its particular case $\nu=0$ using the isomorphisms (5.7.2). For the proof of statement (ii) with $\nu=0 \mathrm{cf}$. [SKK] and [9].

(iii) By (ii) there is an isomorphism 


$$
\left.\left.\hat{\mathcal{E}}_{\left\langle z_{n} / Y \mu\right\rangle}\right|_{X_{n}^{\prime}} \underset{\sim}{\sim} \hat{\mathcal{E}}_{\left\langle X_{n} / Y_{\mu}\right\rangle}\right|_{X_{n} \backslash Y \mu \nu}
$$

associated to the change of coordinates introduced in Lemma 5.8. We define (5.7.2) as the composition of (5.7.5) with the inverse of (5.7.4).

Q.E.D.

Lemma 5.8. Let $X$ be a complex manifold and $Y$ a divisor with normal crossings of $X$. Let $U$ be an open set of $X$ and let $\left(x_{1}, \cdots, x_{n}\right),\left[\left(\tilde{x}_{1}, \cdots, \tilde{x}_{n}\right)\right]$ be $a$ system of local coordinates for $X$ on $U$ such that $Y \cap U=\left\{x_{1} \cdots x_{\nu}=0\right\}\left[=\left\{\tilde{x}_{1} \cdots\right.\right.$ $\left.\tilde{x}_{\tilde{\nu}}=0\right\}$ ] for a certain integer $\nu[\tilde{\nu}]$. Let $\varepsilon[\tilde{\varepsilon}]$ be an imbedding of $U$ into a copy $X_{n}\left[\tilde{X}_{n}\right]$ such that $\varepsilon^{-1}\left(Y_{\nu}\right)=\tilde{\varepsilon}^{-1} Y_{\tilde{\nu}}=Y_{\nu}$. Let $V[\tilde{V}]$ be the image of $\varepsilon[\tilde{\varepsilon}]$. Let $\pi[\tilde{\pi}]$ be the canonical projection of $T^{*}\left\langle X_{n} / Y_{\nu}\right\rangle$ onto $X_{n}\left[T^{*}\left\langle\tilde{X}_{n} / Y_{\tilde{\nu}}\right\rangle\right.$ onto $\left.\tilde{X}_{n}\right]$. There is a canonical isomorphism

$$
\left.\pi^{-1} \mathscr{D}_{X_{n}}\left\langle Y_{\nu}\right\rangle\right|_{V} \underset{\sim}{\rightarrow} \tilde{\pi}^{-1} \mathscr{D}_{\tilde{X}_{n}}\left\langle Y_{\tilde{\nu}}\right\rangle \mid \tilde{V}
$$

Moreover there is one and only one isomorphism

$$
\left.\varepsilon_{\left\langle X_{n} / Y_{\nu}\right\rangle}\right|_{V} \underset{\rightarrow}{\sim} \mathcal{E}_{\left\langle\tilde{X}_{n} / Y \tilde{v}\right\rangle} \mid \tilde{V}
$$

that extends (5.8.1).

Proof. The existence follows from Proposition 5.7. The uniqueness follows from the fact that the Lemma is true when $\nu=\tilde{\nu}=0$ and from Lemma 5.7.1. For the prove in the case $\nu=\tilde{\nu}=0 \mathrm{cf}$. [SKK] or [15]. Q.E.D.

Let $X$ be a complex manifold and $Y$ be a divisor with normal crossings of $X$. We can cover $X$ with copies of open sets $X^{(i)}$ of $C^{n}$ such that there is an integer $\nu_{i}$ verifying $X^{(i)} \cap Y=\left\{x_{\mathrm{I}}^{(i)} \cdots x_{v_{i}}^{(i)}=0\right\}$. We can glue the sheaves $\mathcal{E}_{\left\langle X^{(i)} / X^{(i)} \cap Y\right\rangle}\left[\hat{\varepsilon}_{\left\langle X^{(i)} / X^{(i)} \cap Y\right\rangle}\right]$ using the morphisms introduced in Lemma 5.8 . Again by Lemma 5.8 the sheaf obtained in this way does not depend of the choices of the open sets, the coordinate systems or the integers $\nu_{i}$.

We will denote it by $\mathcal{E}_{\langle X / Y\rangle}\left[\hat{\mathcal{E}}_{\langle X / Y\rangle}\right]$. We call $\mathcal{E}_{\langle X / Y\rangle}\left[\hat{\mathcal{E}}_{\langle X / Y\rangle}\right]$ the sheaf of [formal] logarithmic microdifferential operators of $X$ with poles along $Y$.

The sheaf $\mathcal{E}_{\langle X / Y\rangle}\left[\hat{\mathcal{E}}_{\langle X / Y\rangle}\right]$ has a canonical structure of sheaf of filtered $\boldsymbol{C}$ algebras and $\operatorname{gr} \hat{\mathcal{E}}_{\langle X / Y\rangle}$ is canonically isomorphic to $\mathcal{O}_{T^{*}\langle X / Y\rangle}^{h}$. We will denote respectively by $\sigma, \sigma_{m}$ the natural morphisms of sheaves of sets [sheaves of $\boldsymbol{C}$ modules]

$$
\begin{gathered}
\sigma: \hat{\mathcal{E}}_{\langle X / Y\rangle} \rightarrow \mathcal{O}_{T *\langle X, Y\rangle}^{h}, \\
\sigma_{m}: \hat{\mathcal{E}}_{\langle X / Y\rangle(m)} \rightarrow \mathcal{O}_{T^{*}\langle X / Y\rangle(m)} .
\end{gathered}
$$

If $P \in \hat{\mathcal{E}}_{\langle X / Y\rangle(m)}, Q \in \hat{\mathcal{E}}_{\langle X / Y\rangle(n)}$ then $[P, Q] \in \hat{\mathcal{E}}_{\langle X / Y\rangle(m+n+1)}$ and 


$$
\sigma_{m+n-1}([P, Q])=\left\{\sigma_{m}(P), \sigma_{n}(Q)\right\} .
$$

Proposition 5.9. (i) There is a canonical isomorphism $\left.\mathcal{E}_{\langle X / Y\rangle}\right|_{X \mid Y} \widetilde{\longrightarrow}$ $\left.\mathcal{E}_{X}\right|_{X \backslash Y}$.

(ii) There is a canonical imbedding of $\pi^{-1} \mathscr{D}_{X}\langle Y\rangle$ into $\mathcal{E}_{\langle X / Y\rangle}$.

(iii) The restriction of $\mathcal{E}_{\langle X / Y\rangle}$ to the zero section of $T^{*}\langle X \mid Y\rangle$ equals $\mathscr{D}_{X}\langle Y\rangle$.

Theorem 5.10. The filtered $\boldsymbol{C}$-Algebras $\mathcal{E}_{\langle X / Y\rangle}$ and $\hat{\mathcal{E}}_{\langle X / Y\rangle}$ have zariskian fibers.

Corollary 5. 1 1. (i) The rings $\mathcal{E}_{\langle X / Y\rangle}$ and $\hat{\mathcal{E}}_{\langle x / Y\rangle}$ are (left and right) noetherian rings.

(ii) The inclusions $\pi^{-1} \mathscr{D}_{X}\langle Y\rangle \hookrightarrow \mathcal{E}_{\langle X / Y\rangle}, \mathcal{E}_{\langle X / Y\rangle} \hookrightarrow \hat{\mathcal{E}}_{\langle X / Y\rangle}$ are flat morphisms.

In order to prove Theorem 5.10 we will now introduce an immersion of the ring of logarithmic microdifferential operators into the ring of microdifferential operators of a higher dimensional manifold.

5.12. Let $X_{n+\nu}$ be a copy of $\mathbb{C}^{n+\nu}$ with coordinates $\left(y_{1}, \cdots, y_{n}, z_{1}, \cdots, z_{\nu}\right)$. Put $X_{n+\nu}^{*}=X_{n+\nu} \backslash\left\{y_{1} \cdots y_{\nu}=0\right\}$. Let $(y, z, \eta, \zeta)$ be the canonical coordinates of $T^{*} X_{n+\nu}^{*}$. For $1 \leq i \leq \nu$ let $\alpha_{i},[\alpha]$ be the action of $\mathbb{C}^{*}\left(\mathbb{C}^{* \nu} \times \mathbb{C}^{\nu}\right)$ in $T^{*} X_{n+\nu}^{*}$ given by

$$
\begin{gathered}
\alpha_{i}\left(t_{i}, y, z, \eta, \zeta\right)=\left(t_{i} y, t_{i}^{-1} z, t_{i}^{-1} \eta, t_{i} \zeta\right) \\
\alpha(t, h, y, z, \eta, \zeta)=\left(t y^{\prime}, y^{\prime \prime}, t^{-1} z, t^{-1} \eta^{\prime}, \eta^{\prime \prime}, \zeta+h\right),
\end{gathered}
$$

where $t=\left(t_{1}, \cdots, t_{v}\right), h=\left(h_{1}, \cdots, h_{v}\right), y=\left(y_{1}, \cdots, y_{n}\right), y^{\prime}=\left(y_{1}, \cdots, y_{v}\right), y^{\prime \prime}=\left(y_{v+1}, \cdots\right.$, $\left.y_{n}\right), z=\left(z_{1}, \cdots, z_{v}\right), \quad \eta^{\prime}=\left(\eta_{1}, \cdots, \eta_{v}\right), \eta^{\prime \prime}=\left(\eta_{\nu+1}, \cdots, \eta_{n}\right), t_{i} y=\left(y_{1}, \cdots, t_{i} y_{i}, \cdots, y_{n}\right)$, $t y^{\prime}=\left(t_{1} y_{1}, \cdots, t_{v} y_{v}\right)$ and so on.

For $1 \leq i \leq \nu$ let

$$
\rho_{i}=z_{i} \partial_{z_{i}}-\zeta_{i} \partial_{\zeta_{i}}-\left(y_{i} \partial_{y_{i}}-\eta_{i} \partial_{\eta_{i}}\right)
$$

be the radial vector field of the $C^{*}$-action $\alpha_{i}$. Define $\psi: T^{*} X_{n+\nu}^{*} \rightarrow T^{*}\left\langle X_{n} / Y_{\nu}\right\rangle$ by:

$$
\begin{aligned}
& \psi^{*} x_{i}=z_{i} y_{i}, \quad \psi^{*} \xi_{i}=y_{i} \eta_{i}, \quad 1 \leq i \leq \nu, \\
& \psi^{*} x_{i}=y_{i}, \quad \psi * \xi_{i}=\eta_{i}, \quad \nu+1 \leq i \leq n .
\end{aligned}
$$

The fibers of $\psi$ are the orbits of the action of $\alpha$. Given a complex number $\lambda$ define $\mathcal{O}_{T^{*} X_{n+\nu}^{*}}^{\prime}(\lambda)=\left\{f \in \mathcal{O}_{T^{*} X_{n+\nu}^{*}}(\lambda): \rho_{i} f=\partial_{\zeta_{i}} f=0,1 \leq i \leq \nu\right\}$. The map $\psi$ induces isomorphisms of sheaves 


$$
\psi_{\lambda}: \mathcal{O}_{T^{*}\left\langle X n / Y_{\nu}\right\rangle}(\lambda) \rightarrow \psi_{* *} \mathcal{O}_{T^{*} X_{n+\nu}^{*}}(\lambda), \quad \lambda \in C .
$$

Let $\hat{\mathcal{E}}_{X_{n+\nu}}^{\prime}$ be the sheaf of the formal microdifferential operators $P \in \hat{\mathcal{E}}_{X_{n+\nu}}$ such that

$$
\left[z_{i} \partial_{z_{i}}-y_{i} \partial_{y_{i}}, P\right]=\left[P, z_{i}\right]=0 \quad \text { for } 1 \leq i \leq \nu .
$$

We will now build an isomorphism of filtered $\boldsymbol{C}$-modules

$$
\Psi: \hat{\mathcal{E}}_{\left\langle X_{n} / Y_{\nu}\right\rangle} \rightarrow \psi_{*} \hat{\mathcal{E}}_{X_{n+\nu}^{*}}
$$

We define $\Psi_{0}:\left.\left.\hat{\mathcal{E}}_{X_{n}}\right|_{X_{n} \backslash Y_{\nu}} \rightarrow \psi_{*} \hat{\mathcal{E}}_{X_{n+\nu}^{*}}\right|_{X_{n} \backslash Y_{\nu}}$ by $\sum_{j} Q_{j} \mapsto \Sigma_{j} Q_{j} \circ \psi$. Let now $\Sigma_{j} P_{j}$ be the total symbol of a section $P$ of $\hat{\varepsilon}_{\left\langle X_{n} \backslash Y_{\nu}\right\rangle}$. If $\sum_{j} Q_{j}$ is the principal symbol of $\left.P\right|_{X_{n} \mid Y_{\nu}}$ as a section of $\mathcal{E}_{X_{n}}$ then by (5.7.3) all the $Q_{j}$ 's have a unique extension $\widetilde{Q}_{j}$ to the domain of $P$. We can now define $\Psi(P)=\Sigma_{j} \widetilde{Q}_{j} \circ \varphi$. We can easily verify that $\Psi_{0}$ is a morphism of filtered $\boldsymbol{C}$-algebras and therefore the same happens with $\Psi$.

Once we prove the morphism $\Psi$ is an isomorphism then Theorem 5.10 will be a consequence of the following Lemma.

Lemma 5.12.1. The sheaves $\mathcal{E}_{X_{n+\nu}^{*}}^{\prime}$ and $\hat{\mathcal{E}}_{X_{n^{*}}^{*}}^{\prime}$ have zariskian fibers.

Proof. Let $\hat{\mathcal{E}}_{X_{n+\nu}^{*}}^{\prime \prime}$ be the sheaf of formal microdifferential operators $P \in$ $\hat{\mathcal{E}}_{X_{n+\nu}^{*}}^{\prime}$ such that $\left[P, z_{j}\right]=0$ for $1 \leq i \leq \nu$. The sheaf $\mathcal{E}_{X_{n+\nu}^{*}}^{\prime \prime}$ has zariskian fibers. (cf. [SKK] and [15]). Fixed $p^{0} \in T^{*} \tilde{X}_{0}$ define, for $0 \leq i \leq \nu$,

$$
E_{l}=\left\{P \in \mathcal{E}_{X_{n_{+}}^{*}}^{\prime \prime} \text { such that }\left[\rho_{i}, P\right]=0 \text { for } 1 \leq i \leq l\right\} .
$$

Lemma 5.12.2. For $0 \leq i \leq \nu$ there is a sub $\boldsymbol{C}$-module $E_{l}^{\prime}$ of $E_{0}$ such that $E_{l} \oplus E_{l}^{\prime}=E_{0}$.

Proof. If $l=0$ the statement is obvious. Suppose $1 \leq l \leq \nu$. There are holomorphic functions $p_{1}, \cdots, p_{n+\nu-1}$, homogeneous of degree 0 , and a holomorphic function $\eta$, homogeneous of degree 1 , such that

$$
y_{i}, 1 \leq i \leq n, z_{i}, 1 \leq i \leq \nu, i \neq l, z_{l} y_{l}, p_{i}, 1 \leq i \leq n+\nu-1, \eta
$$

is a system of local coordinates for $X_{n+\nu}^{*}$ in a neighbourhood of $p^{0}$. Relatively to this system of coordinates

$$
\rho_{l}=-y_{l} \partial_{y l}, \quad \rho=\eta_{l} \partial_{\eta l} .
$$

Given $P \in E_{l-1}$ there are holomorphic functions $a_{i}, i \in Z$, such that $P=\sum_{i} a_{i} \eta^{i}$ 
and $\rho a_{i}=\rho_{k} a_{i}=0$ for $1 \leq k \leq l-1, i \in \mathbb{Z}$. For each $i$ there are holomorphic functions $a_{i j}$ such that

$$
a_{i}=\sum_{j \geq 0} a_{i j}\left(y_{l}-y_{l}\left(p^{0}\right)\right)^{j}
$$

and $\rho_{l} a_{i j}=0$ for all $j$. Therefore $E_{l-1}=E_{l} \oplus\left(y_{l}-y_{l}\left(p^{0}\right)\right) E_{l-1}$. We take $E_{l}^{\prime}=$ $E_{l-1}^{\prime} \oplus\left(y_{l}-y_{l}\left(p^{0}\right)\right) E_{l-1}$. Q.E.D.

Lemma 5.12.1 is a consequence of Lemma 5.12.2 for $l=\nu$ and the fact that if $I$ is a left ideal (right ideal) of $\hat{\mathcal{E}}_{X_{n+\nu}^{*}, p^{0}}^{\prime *}$ then $I \oplus E_{\nu}^{\prime}$ is a left ideal (right ideal) of $\hat{\varepsilon}_{X_{n+\nu}^{*}, p^{0}}$.

Q.E.D.

Theorem 5.13. Let $k$ be a field and $A$ a (left) noetherian $k$-algebra. Suppose that there is a both side ideal I of $A$ such that $[A, A] \subset I,[A, I] \subset I^{2}$ and $I^{N}$ $=0$ for $N \gg 0$. Then, given a both side ideal $\Re$ of $A$ the ring $\bigoplus_{k \geq 0} \Re^{k}$ is noetherian.

Corollary 5.14. If for any $a \in \mathfrak{N} 1-a$ is invertible in $A$ then the filtration of $A$ defined by $F_{k} A=\Re^{-k}$ if $k \leq 0, F_{k} A=A$ if $k \geq 0$ is zariskian.

Proof of the Theorem 5.13. There are elements $u_{1}, \cdots, u_{l}$ of $A$ such that $\Re=\sum_{i=1}^{l} A u_{i}$. Let $\mathcal{G}$ be the Lie algebra over $k$ generated by the indeterminates $T_{1}, \cdots, T_{l}$, and verifying the relations $(a d \mathcal{G})^{N} \mathcal{G}=0$. We define recursively a family $\left(\mathscr{N}_{k}\right)_{k \geq 0}$ of left ideals of $A$ by $\Re_{0}=A, \Re_{k+1}=A\left[\Re, \mathscr{N}_{k}\right] \subset I^{k}$. Let $B$ be the Lie algebra of the derivations of $\bigoplus_{k \geq 0} \Re_{k} T^{k}$, where $T$ is an indeterminate.

Let $\varphi: \mathcal{G} \rightarrow \mathscr{B}$ be the morphism of Lie algebras defined by $\varphi\left(T_{i}\right)=\left[u_{i}, *\right] T$. Let $U_{\varphi}(B)$ be the $k$-algebra generated by $\mathcal{G}$ and $A$ with fundamental relations

$$
[x, a]=\varphi(x)(a), \quad x \in \mathcal{G}, a \in A .
$$

We have an epimorphism

$$
s: U_{\varphi}(B) \rightarrow \oplus_{k \geq 0} \Re^{k} T^{k}
$$

defined by $s(a)=a$ for $a \in A$ and $s\left(T_{j}\right)=u_{j} T$ for $1 \leq j \leq l$.

It is therefore enough to show that $U_{\varphi}(B)$ is a noetherian ring. Let $U(\mathcal{G})$ be the universal enveloping algebra of $\mathcal{G}$. We introduce a structure of right $A$-module in $A \otimes_{k} U(\mathcal{G})$ by

$$
(1 \otimes x) a:=a \otimes x+\varphi(x)(a) \otimes 1 .
$$

We endow in this way $A \otimes_{k} U(\mathcal{G})$ with a structure of ring for which $A \otimes_{k} U(\mathcal{G})$ and $U_{\varphi}(\mathcal{G})$ are isomorphic. The canonical filtration of $U(\mathcal{G})$ induces a filtra- 
tion on $A \otimes_{k} U(\mathcal{G})$. The associated graduated ring is an epimorphic image of $A\left[T_{1}, \cdots, T_{l}\right]$.

Q.E.D.

Corollary 5.15. Let $A$ be a k-algebra with a zariskian filtration. Let $\eta$ be $a$ both side ideal of $A_{0}$ verifying the conditions of Corollary 5.14. Let $B$ be a $k$-subalgebra of $A$ and consider in $B$ the induced filtration. If $B$ contains an element of $A$, invertible of order 1 , and $\cap_{k}\left(B_{0}+\Upsilon^{k}\right)$ equals $A_{0}$ then $B$ equals $A$.

Proof. Given a nonnegative integer $l$ put $\Re_{l}=\left(\mathscr{l}+A_{l}\right) / A_{l}$. Then by Corollary 5.14 the filtration of $A / A_{l}$ given by $F_{k}\left(A / A_{l}\right)=A / A_{l}$ if $k \geq 0$, $F_{k}\left(A / A_{l}\right)=\Re_{l}^{-k}$ if $k \leq 0$ is zariskian. Consider in $\left(B_{0}+A_{l}\right) / A_{l}$ the induced filtration. Since

$$
0 \rightarrow \operatorname{gr}\left(\left(B_{0}+A_{l}\right) / A_{l}\right) \rightarrow \operatorname{gr}\left(A_{0} / A_{l}\right)
$$

is exact then $\left(B_{0}+A_{l}\right) / A_{l}$ equals $A_{0} / A_{l}$. Therefore $B_{0}+A_{l}$ equals $A_{0}$. Since the filtration of $A$ is zariskian then $B_{0}$ equals $A_{0}$.

Q.E.D.

We will now finish the proof of Theorem 5.10. Choose $p^{0} \in T^{*} X_{n+\nu}^{*}$. Put

$$
A=\hat{\mathcal{E}}_{X_{n+\nu}^{*}, p^{0}}, \quad B=\operatorname{Im}\left(\Psi_{p^{0}}: \hat{\mathcal{E}}_{\left\langle X_{n} / Y_{\nu}\right\rangle, \pi\left(p^{0}\right)} \rightarrow \hat{\mathcal{E}}_{X_{n+\nu}^{*}, p^{0}}\right) .
$$

Let $m$ be the maximal ideal of gr $A_{0}$ and put $\Re=\sigma_{0}^{-1} m$.

If $p^{0}$ is in the zero section the surjectivity of $\Psi_{p^{0}}$ is trivial. We can therefore suppose that $p^{0} \in \stackrel{\circ}{T}^{*} X_{n+\nu}^{*}$. Put

$$
w_{i}=y_{i} z_{i}, w_{i+n}=z_{i} \text { for } 1 \leq i \leq \nu, w_{i}=y_{i} \text { for } \nu+1 \leq i \leq n .
$$

We can choose $p_{1}, \cdots, p_{n+\nu-1} \in \mathcal{O}_{T^{k} X_{n+\nu, p^{0}}^{k}}(0), \eta \in \mathcal{O}_{T^{*} X_{n+\nu}^{*}, p^{0}(1)}$ invertible, $P_{1}, \cdots$, $P_{n+\nu-1} \in B_{0}, P \in B_{1}$ such that $\sigma_{0}\left(P_{i}\right)=p_{i}, 1 \leq i \leq n+\nu-1, \sigma_{1}(P)=\eta$ and $(w, p, \eta)$ is a system of local coordinates for $T^{*} X_{n+\nu}^{*}$ in some neighbourhood of $p^{0}$. Moreover $B$ contains an invertible element of $A$ of order 1 . Given $Q \in A_{0}$ and a nonnegative integer $k$ there is a polynomial function $\Pi$ of

$$
w_{i}-w_{i}\left(p^{0}\right), 1 \leq i \leq n+\nu, p_{i}-p_{i}\left(p^{0}\right), 1 \leq i \leq n+\nu-1,
$$

such that $\sigma(Q)-\Pi \in m^{k}$. Therefore by the Corollary $5.15 B$ equals $A$.

This ends the proof of Theorem 5.10.

\section{§6. Division Theorems}

We will associate to each logarithmic microdifferential operator $P$ the 
C-linear isomorphism $a d_{P}$ of $\mathcal{E}_{\langle X / Y\rangle}$ defined by $a d_{P}(Q)=[P, Q]$.

Theorem 6.1. Let $P$ be a logarithmic microdifferential operator defined in a neighbourhood of $\left(x^{0}, \xi^{0}\right) \in T^{*}\langle X \mid Y\rangle$. Assume that $\partial_{\xi_{\mu}}^{j} \sigma(P)$ is zero at $\left(x^{0}, \xi^{0}\right)$ for $0 \leq j \leq l-1$ and different from zero for $j=1$. Then for any section $Q$ of $\mathcal{E}_{\langle x / Y\rangle}$ defined in a neighbourhood of $\left(x^{0}, \xi^{0}\right)$ there are unique sections $S$ and $R$ of $\varepsilon_{\langle x / Y\rangle}$ defined in a neighbourhood of $\left(x^{0}, \xi^{0}\right)$ such that

$$
Q=S P+R \text { and } a d_{x_{\mu}}^{l}(R)=0 .
$$

Moreover ord $R \leq$ ord $Q$.

Remark 6.2. (i) We notice that $a d_{x_{y}}^{k} R=0$ iff there are microdifferential operators $R^{(0)}, \cdots, R^{(k-1)}$ such that $a d_{x y} R^{(i)}=0$ for $1 \leq i \leq l-1$ and $R=$ $\sum_{i=0}^{k-1} R^{(i)} \delta_{x_{\mu}}^{i}$.

(ii) With the same hypothesis there exists also $\tilde{S}$ and $\widetilde{R}$ such that $Q=P \widetilde{S}+\widetilde{R}$ and $a d_{x_{\mu}}^{k}=0$. Moreover $\widetilde{X}$ and $\widetilde{R}$ are unique and ord $\widetilde{R} \leq$ ord $Q$.

(iii) We can interchange $x_{\mu}$ adn $\delta_{x_{\mu}}$ in the statement of the theorem.

(iv) If an operator $A$ commutes with $P, Q$ and $x_{\mu}$ then $A$ also commutes with $S$ and $R$.

Proof. We will admit the Theorem with the additional hypothesis $Y=\phi$ (cf. [SKK] or [15]). We will use the constructions introduced during the proof of Theorem 5.10 .

We will consider in $X_{n+v}^{*}$ the system of coordinates (5.15.1).

Let $U$ be an open neighbourhood of $\left(x^{0}, \xi^{0}\right)$ in which $P$ and $Q$ are defined. Put $\tilde{P}=\Psi(P), \tilde{Q}=\Psi(Q)$. Given $\left(y^{0}, z^{0}, \eta^{0}, \zeta^{0}\right) \in T^{*} X_{n+\nu}^{*}$ if $\psi\left(y^{0}, z^{0}, \eta^{0}, \zeta^{0}\right)=$ $\left(x^{0}, \xi^{0}\right)$ then $\partial_{w_{\mu}}^{j} \sigma(\widetilde{P})\left(y^{0}, z^{0}, \eta^{0}, \zeta^{0}\right)$ equals zero for $0 \leq j \leq l-1$ and is different from zero for $j=l$. Then there are unique microdifferential operators $\tilde{S}$ and $\widetilde{R}$ defined in a neighborhood of $\left(y^{0}, z^{0}, \eta^{0}, \zeta^{0}\right)$ such that $\tilde{Q}=\tilde{S} \widetilde{P}+\widetilde{R}$ and $a d_{w_{\mu}}^{l} \widetilde{R}=0$. By Remark $6.2 z_{i}$ and $\rho_{i}$ commute with $\widetilde{R}$ and $\widetilde{S}$ for $1 \leq i \leq \nu$.

Hence there are logarithmic microdifferential operators $R, S$, defined in a neighbourhood of $\left(x^{0}, \xi^{0}\right)$, such that $\Psi(R)=\widetilde{R}, \Psi(S)=\widetilde{S}$. We can easily verify that they have the desired properties.

Q.E.D.

Corollary 6.3. With the same hypothesis on $P$ there exists an invertible microdifferential operator $A$ and a microdifferential operator $W$ such that

$$
P=A W, \quad W=\delta_{x_{\mu}}^{l}+\sum_{j=0}^{l-1} R_{j} \delta_{x_{\mu}}^{j}
$$

and $\left[x_{\mu}, R_{j}\right]=0$ for $0 \leq j \leq l-1$, ord $W \leq l$. 
We will now use the Division Theorem to study the both side ideals of $\varepsilon_{\langle X / Y\rangle}$

Let $A$ be an associative ring with identity. We say that a proper both side ideal $I$ of $A$ is prime if given two both side ideals $I_{1}, I_{2}$ of $A, I_{1} I_{2}=I$ implies $I=I_{1}$ or $I=I_{2}$. We notice that a both side ideal $I$ of $A$ is prime if and only if $a A b \in I$ implies $a \in I$ or $b \in I$ for any $a, b \in A$. A maximal both side ideal is always prime.

Definition 6.4. Let $\mathcal{A}$ be a coherent sheaf of rings over a topological space $X$. We say that a coherent ideal [both side ideal] $\mathcal{G}$ of $\mathcal{A}$ is proper [prime, maximal] along a subset $Y$ of $X$ if the stalk $\mathcal{G}_{x}$ of $\mathcal{G}$ is a proper [prime, maximal] ideal of $\mathcal{A}_{x}$ for any $x \in Y$ and $\mathcal{I}_{x}$ equals $\mathcal{A}_{x}$ for $x \notin Y$.

Remark 6.5. The following relations are a consequence of the Division Theorem for $1 \leq i \leq \nu$.

$$
\begin{aligned}
\left(x_{i}\right)=\varepsilon_{\left\langle X_{n} / Y_{\nu}\right\rangle} x_{i}=x_{i} & \varepsilon_{\left\langle X_{n} / Y_{\nu}\right\rangle}, \\
{\left[\delta_{x_{i}}, \varepsilon_{\left\langle X_{n} / Y_{\nu}\right\rangle}\right] } & \subset\left(x_{i}\right), \\
\left(\delta_{x_{i}}+\lambda\right) & =\varepsilon_{\left\langle X_{n} / Y_{\nu}\right\rangle x_{i}}+\varepsilon_{\left\langle X_{n} / Y_{\nu}\right\rangle}\left(\delta_{x_{i}}+\lambda\right) \\
& =x_{i} \varepsilon_{\left\langle x_{n} / Y_{\nu}\right\rangle}+\left(\delta_{x_{i}}+\lambda\right) \varepsilon_{\left\langle X_{n} / Y_{\nu}\right\rangle} .
\end{aligned}
$$

Theorem 6.6. (i) For $1 \leq i \leq \nu\left(x_{i}\right)$ is the only both side ideal of $\mathcal{E}_{\left\langle X_{n} / Y_{\nu}\right\rangle}$ that is prime along $\left\{x_{i}=0\right\}$.

(ii) For $1 \leq i \leq \nu$ the both side ideals $\left(\delta_{x_{i}}+\lambda\right), \lambda \in C$, are the only both side ideals of $\mathcal{E}_{\left\langle x_{n} / Y_{\nu}\right\rangle}$ that are maximal along $\left\{x_{i}=\xi_{i}=0\right\}$.

Proof. Let's fix an integer $i, 1 \leq i \leq \nu$. The fact that $\left(x_{i}\right)$ is a prime ideal along $\left\{x_{i}=0\right\}$ is an obvious consequence of the Division Theorem and Remark 6.5 .

Let $\mathcal{G}$ be a prime ideal along $\left\{x_{i}=0\right\}$. Suppose that $x_{i} \notin \mathcal{G}$. Given a non zero section $P$ of $\mathcal{G}$ there is a holomorphic function $f$ and a positive integer $m$ such that $\sigma(P)=f x_{i}^{m}$ and $f$ does not vanish along $\left\{x_{i}=0\right\}$. Suppose $m \geq 2$. Then there are logarithmic microdifferential operators $A_{0}, A_{1}, \cdots, A_{m}$ such that

$$
P=\sum_{j=0}^{m} A_{j} x_{i}^{j}, \quad\left[\delta_{x_{i}}, A_{j}\right]=0,
$$

and ord $A_{j}<$ ord $A_{m}$ for $0 \leq j \leq m-1$.

Then $\left[\delta_{x_{i}}, P\right]=\left(\left[\delta_{x_{i}}, A_{n}\right] x_{i}^{m-1}+\sum_{j=1}^{m} j A_{j} x_{i}^{j-1}\right) x_{i}$. Hence $\left[\delta_{x_{i}}, P\right] x_{i}^{-1} \in \mathcal{G}$ and $\mathfrak{S}\left(\left[\delta_{x_{i}}, P\right] x_{i}^{-1}\right)=f^{\prime} x_{i}^{m-1}$ for some holomorphic function $f^{\prime}$ that does not vanish along $\left\{x_{i}=0\right\}$. We can therefore suppose $m=1$. Repeating the reasoning 
above with $m=1$ we conclude that $A_{1}+\left[\delta_{x_{i}}, A_{1}\right] \in \mathcal{J}$. Since this logarithmic microdifferential operator is invertible in a generic point of $\left\{x_{\dot{i}}=0\right\}$ then the assumption $x_{8} \notin \mathcal{G}$ leaded us into a contradiction. We can now conclude from the Division Theorem that $\mathcal{G}$ equals $\left(x_{\dot{\xi}}\right)$.

(ii) Let $\mathcal{G}$ be a maximal ideal along $\left\{x_{i}=\xi_{i}=0\right\}$ and $P$ a local non zero section of $\mathcal{G}$ defined in a neighbourhood of a point in the residual set $\left\{x_{\dot{\varepsilon}}=\xi_{\dot{z}}=0\right\}$. The logarithmic microdifferential operators $\left[\delta_{x_{i}}, P\right]$ and $\left[P, x_{i}\right]$ cannot vanish simultaneously otherwise $P$ would be invertible in a generic point of $\left\{x_{i}=\xi_{i}=\right.$ $0\}$. Since $\left[\delta_{x_{i}}, P\right],\left[P, x_{i}\right] \in\left(x_{i}\right)$ we can repeat the argument of the proof of (i) and conclude that $\left(x_{i}\right) \subset \mathcal{G}$.

Choose now $P \in \mathcal{G} \backslash\left(x_{i}\right)$. We can suppose that, at a generical point of $\left\{x_{i}=\xi_{i}=0\right\}, \sigma(P)=\xi_{i}^{m}$. Hence we can suppose that, at a generical point of $\left\{x_{i}=\xi_{i}=0\right\}, P=\delta_{x_{i}}^{m}+\sum_{j=0}^{m-1} R_{j} \delta_{x_{i}}^{j}$, where $\left[R_{j}, x_{i}\right]=\left[\delta_{x_{i}}, R_{j}\right]=0$ for $1 \leq i \leq m-1$.

If the logarithmic microdifferential operators $R_{j}$ are constant then there are complex numbers $\lambda_{1}, \cdots, \lambda_{m}$ such that

$$
P=\mathbb{I}_{j=1}^{m}\left(\delta_{x_{i}}+\lambda_{j}\right) .
$$

Otherwise let $m_{0}$ be the highest $j$ such that $R_{j}$ is not constant. Then $n>1$ and there is a microdifferential operator $S$ such that $\left[S, R_{m_{0}}\right] \neq 0$ and $\left[\delta_{x_{i}}, S\right]=\left[S, x_{i}\right]$ $=0$. The integer $m_{0}$ must be positive. There is a microdifferential operator $U$ defined in a generical point of the support of $[S, P]$ such that $\sigma(U[S, P])=$ $\xi_{i}^{m_{0}}$ and

$$
U[S, P]=\delta_{x_{i}}^{m_{0}}+\sum_{j=0}^{m_{0}-1} R_{j}^{\prime} \delta_{x_{i}}^{j},
$$

where $R_{j}^{\prime}$ verifies the same conditions as the operator $R_{j}$ considered above. After repeating this operation a finite number of times we will obtain an operator $P$ verifying (6.6.2). Therefore there is a $\lambda$ such that $\left(\delta_{x_{i}}+\lambda\right) \subset \mathcal{G}$ in a generic point of $\left\{x_{\dot{\varepsilon}}=\xi_{i}=0\right\}$. We conclude now from the Division Theorem that $\mathcal{G} \subset\left(\delta_{x_{i}}+\lambda\right)$.

Q.E.D.

\section{§7. Quantized Contact Transformations}

Definition 7.1. Let $X[\tilde{X}]$ be a complex manifold and $Y[\tilde{Y}]$ a divisor with normal crossings of $X[\tilde{X}]$. Let $U$ be an open set of $T^{*}\langle X / Y\rangle$ and $\varphi: U \rightarrow$ $T^{*}\langle\tilde{X} / \tilde{Y}\rangle$ be a symplectic transformation, homogeneous outside the zero section. We say that a filtered $\mathbb{C}$-algebras isomorphism

$$
\Phi:\left.\varphi^{-1} \mathcal{E}_{\langle\tilde{X} / \tilde{Y}\rangle} \rightarrow \mathcal{E}_{\langle X / Y\rangle}\right|_{U}
$$


is a quantization of $\varphi$ if the following diagram commutes for any integer $m$.

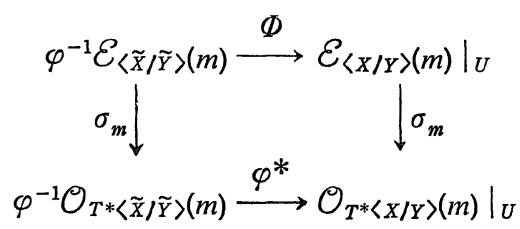

We call the pair $(\varphi, \Phi)$ a quantized contact transformation.

Definition 7.2. Let $X$ be a complex manifold and $Y$ be a divisor with normal crossings of $Y$. We say that a family

$$
\left(P_{1}, \cdots, P_{n}, Q_{1}, \cdots, Q_{n}\right)
$$

of logarithmic microdifferential operators defined in an open set $U$ of $T^{*}\langle X \mid Y\rangle$ is a system of quantized contact coordinates for $\mathcal{E}_{\langle X / Y\rangle}$ on $U$ if

(i) $P_{1}, \cdots, P_{n}$ have order $1, Q_{1}, \cdots, Q_{n}$ have order 0 .

(ii) The principal symbols of the logarithmic microdifferential operators (7.2.1) define a system of homogeneous logarithmic symplectic coordinates for the homogeneous logarithmic symplectic manifold $U$.

(iii) The following commutation relations hold:

$$
\begin{gathered}
{\left[P_{k}, Q_{j}\right]= \begin{cases}\delta_{j k} Q_{j} & \text { if } \sigma\left(\left[P_{j}, Q_{j}\right]\right)=\sigma\left(Q_{j}\right) \\
\delta_{j k} & \text { if } \sigma\left(\left[P_{j}, Q_{j}\right]\right)=1,\end{cases} } \\
{\left[P_{i}, P_{j}\right]=\left[Q_{i}, Q_{j}\right]=0 \quad 1 \leq i, j \leq n}
\end{gathered}
$$

Theorem 7.3. Let $X$ be a complex manifold and $Y$ a divisor with normal crossings of $X$. Let $\left(P_{1}, \cdots, P_{n}, Q_{1}, \cdots, Q_{n}\right)$ be a system of quantized contact coordinates for $\mathcal{E}_{\langle X / Y\rangle}$ on $U$. Let $\nu$ be the only integer such that $\left[P_{k}, Q_{k}\right]=Q_{k}$ for $1 \leq i \leq \nu$ and $\left[P_{k}, Q_{k}\right]=1$ for $\nu+1 \leq i \leq n$. Let $X_{n}$ be a copy of $\mathbb{C}^{n}$ with coordinates $\left(x_{1}, \cdots, x_{n}\right)$ and let $(x, \xi)$ be the associated system of canonical coordinates of $T^{*}\langle X \mid Y\rangle$. Let $\varphi: U \rightarrow T^{*}\left\langle X_{n} / Y_{\nu}\right\rangle$ be the contact transformation defined by $\varphi^{*} x_{i}=\sigma\left(Q_{i}\right), \varphi^{*} \xi_{i}=\sigma\left(P_{i}\right)$ for $1 \leq i \leq n$.

Then there is one and only one quantization $\Phi$ of the contact transformation $\varphi$ such that

$$
\Phi\left(x_{i}\right)=Q_{i}, \quad \Phi\left(\delta_{x_{i}}\right)=P_{i}, \quad 1 \leq i \leq n .
$$

Proof. We can identify $U$ with its image by $\varphi$. We are then reduced to prove the following statement.

(7.3.1) Let $U$ be an open set of $T^{*}\left\langle X_{n} / Y_{\nu}\right\rangle$ and $\left(P_{1}, \cdots, P_{n}, Q_{1}, \cdots, Q_{n}\right)$ be a system of quantized contact coordinates for $\mathcal{E}_{\left\langle X_{n} / Y_{\nu}\right\rangle}$ on $U$ such that $\sigma\left(Q_{i}\right)=$ 
$x_{i}, \sigma\left(P_{i}\right)=\xi_{i}, 1 \leq i \leq n$. Then there is an automorphism $\Phi$ of $\left.\varepsilon_{\left\langle x_{n} / Y_{y}\right\rangle}\right|_{U}$ such that $\Phi\left(x_{i}\right)=Q_{i}, \Phi\left(\delta_{x_{i}}\right)=P_{i}, 1 \leq i \leq n$

We will first prove the uniqueness. Let $\Phi, \Phi^{\prime}$ be two quantizations of $\mathrm{id}_{U}$ and let $p^{0}$ be a point of $U$. Put $A=\tilde{\mathcal{E}}_{\left.\left\langle X_{n} / Y\right\rangle\right\rangle, p^{0}}$ and $B=\left\{P \in A: \Phi(P)=\Phi^{\prime}(P)\right\}$. Then $B$ equals $A$ by Theorem 5.10 and Corollary 5.15 .

We will now prove the existence. We assume the Theorem proved in the case $\nu=0$ (cf. [SKK] or [15]). By the Division Theorem there are unique microdifferential operators $A_{i}, B_{i}, C_{i}, D_{i}, 1 \leq i \leq \nu$, such that

$$
\begin{array}{ll}
Q_{i}=A_{i} x_{i}+D_{i}, & {\left[\delta_{x_{i}}, D_{i}\right]=0,} \\
P_{i}=\delta_{x_{i}}+B_{i} x_{i}+C_{i}, & {\left[\delta_{x_{i}}, C_{i}\right]=0 .}
\end{array}
$$

Moreover $A_{i}$ is invertible of order $0, B_{i}, C_{i} \in \mathcal{E}_{(0)}, D_{i} \in \mathcal{E}_{(-1)}$. We conclude from the commutation relations 7.2 (iii) and Remark 6.5 that $\left[C_{i}, D_{i}\right] \equiv D_{\dot{i}}$ $\left(\bmod \left(x_{i}\right)\right)$. Therefore $\left[C_{i}, D_{i}\right]=D_{i} . \quad$ Henceforth $D_{i}=0$.

We will first prove the (7.3.1) under the following assumption.

(7.3.3) $\quad C_{i}$ is constant for $1 \leq i \leq \nu$.

We will use again the construction introduced in the proof of Theorem 5.10. For $1 \leq i \leq \nu$ put

$$
\begin{array}{rlrl}
\widetilde{P}_{i} & =\Psi\left(A_{i}\right)^{-1}\left(\partial_{y_{i}}+z_{i} \Psi\left(B_{i}\right)+\frac{1}{y_{i}} C_{i}\right), & \\
\tilde{Q}_{i} & =y_{i} \Psi\left(A_{i}\right), & & \\
R_{n+i} & =\partial_{z_{i}}+y_{i} \Psi\left(B_{i}\right), & S_{n+i}=z_{i}, & 1 \leq i \leq \nu, \\
\tilde{P}_{i} & =\Psi\left(P_{i}\right), & \tilde{Q}_{i}=\Psi\left(Q_{i}\right), \nu+1 \leq i \leq n .
\end{array}
$$

Then $(\tilde{P}, R, \tilde{Q}, S)$ is a system of quantized contact coordinates for $T^{*} X_{n+\nu}^{*}$ on $\psi^{-1}(U)$. Let $\tilde{\Phi}$ be the quantization of $\operatorname{id}_{\psi^{-1}(U)}$ such that

$$
\begin{array}{ll}
\tilde{\Phi}\left(y_{i}\right)=\tilde{Q}_{i}, \quad \tilde{\Phi}\left(\partial_{y_{i}}\right)=\tilde{Q}_{i}, 1 \leq i \leq n, \\
\tilde{\Phi}\left(z_{i}\right)=S_{i}, \quad \tilde{\Phi}\left(\partial_{z_{i}}\right)=R_{i}, 1 \leq i \leq \nu .
\end{array}
$$

Since $\tilde{\Phi}\left(y_{i} \partial_{y_{i}}-z_{i} \partial_{z_{i}}\right)=y_{i} \partial_{y_{i}}-z_{i} \partial_{z_{i}}+C_{i}$ for $1 \leq i \leq \nu, \tilde{\Phi}$ induces an automorphism of $\left.\mathcal{E}_{x_{n}^{*}}\right|_{\psi^{-1}(U)}$ and the Theorem is proved under the assumption (7.3.3).

Example 7.4. Given $\lambda \in \mathbb{C}^{\nu}$ there is one and only one quantization $\Phi_{\lambda}$ of $i d_{T^{*}\langle x / Y\rangle}$ such that 


$$
\begin{aligned}
\Phi_{\lambda}\left(x_{i}\right) & =x_{i}, & & 1 \leq i \leq n, \\
\Phi_{\lambda}\left(\delta_{x_{i}}\right) & =\delta_{x_{i}}+\lambda_{i}, & & 1 \leq i \leq \nu, \\
\Phi_{\lambda}\left(\delta_{x_{i}}\right) & =\delta_{x_{i}}, & & \nu+1 \leq i \leq n .
\end{aligned}
$$

Actually the system of quantized contact coordinates $\left(x_{1}, \cdots, x_{n}, \delta_{x_{i}}+\lambda_{1}, \cdots, \delta_{x_{y}}+\right.$ $\lambda_{\nu}, \delta_{x_{\nu+1}}, \cdots, \delta_{x_{n}}$ ) verifies (7.3.3).

Put $A=\mathcal{E}_{\left\langle x_{n} / Y_{\nu}\right\rangle, p^{0}}$ and $B=\left\{P \in A:\left[\delta_{x_{i}}+A_{i} x_{i}+C_{i}, P\right] \in\left(x_{i}\right)\right\}$. Once more $B$ equals $A$ by Theorem 5.10 and Corollary 5.15.

Therefore $\left[C_{i}, x_{j}\right]=\left[C_{i}, \delta_{x_{j}}\right]=0$ for $1 \leq j \leq n, j \neq i$. We conclude from the commutation relations that $\left[C_{i}, A_{i} x_{i}\right] \in\left(x_{i}^{2}\right)$. If ord $C_{i} \leq l$

$$
\sigma\left(A_{i}\right) \frac{\partial \sigma_{l}\left(C_{i}\right)}{\partial \xi_{i}}+\left\{\sigma_{l}\left(C_{i}\right), \sigma\left(A_{i}\right)\right\} \in\left(x_{i}\right) .
$$

Since $\sigma\left(C_{i}\right)$ only depends of $\xi_{i}$ and $\sigma\left(A_{i}\right)$ is invertible then $\partial_{\xi_{i}} \sigma_{l}\left(C_{i}\right)=0$. Therefore

$$
\text { the symbol of } C_{i} \text { is constant. }
$$

Put $\lambda_{i}=\sigma_{0}\left(C_{i}\right)$ for $1 \leq i \leq \nu$ and $\lambda=-\left(\lambda_{1}, \cdots, \lambda_{\nu}\right)$. By Example 7.4 the family of logarithmic microdifferential operators

$$
\left(\Phi_{\lambda}\left(Q_{1}\right), \cdots, \Phi_{\lambda}\left(Q_{n}\right), \Phi_{\lambda}\left(P_{1}\right), \cdots, \Phi_{\lambda}\left(P_{n}\right)\right)
$$

constitute a system of quantized contact coordinates for $T^{*}\left\langle X_{n} / Y_{\nu}\right\rangle$ on $U$. Moreover $\Phi_{\lambda}\left(P_{i}\right)$ belongs to $\left(\delta_{x_{i}}\right)+\mathcal{E}_{\left\langle x_{n} / Y_{\nu}\right\rangle}(-1)$ for $1 \leq i \leq \nu$. Therefore by (7.4.1) $\Phi_{\lambda}\left(P_{i}\right) \in\left(\delta_{x_{i}}\right)$. Hence $P_{i}$ verifies (7.3.3).

This ends the proof of Theorem 7.3.

Corollary 7.5. Let $\tilde{X}$ be a complex manifold and $\tilde{Y}$ a divisor with normal crossings of $\tilde{X}$. Let $\tilde{U}$ be an open set of $T^{*}\langle\tilde{X} / \tilde{Y}\rangle$ and $(\tilde{Q}, \tilde{P})$ a system of quantized contact coordinates for $\mathcal{E}_{\langle\tilde{X} / \tilde{Y}\rangle}$ on $\tilde{U}$.

Let $\varphi: \widetilde{U} \rightarrow U$ be the only canonical transformation such that

$$
\varphi^{*}\left(\sigma\left(P_{i}\right)\right)=\sigma\left(\widetilde{P}_{i}\right), \varphi^{*} \sigma\left(Q_{i}\right)=\sigma\left(\tilde{Q}_{i}\right), \quad 1 \leq i \leq n .
$$

Then there is one and only one quantization $\Phi$ of $\varphi$ such that

$$
\Phi\left(P_{i}\right)=\widetilde{P}_{i}, \Phi\left(Q_{i}\right)=\tilde{Q}_{i}, \quad 1 \leq i \leq n .
$$

\section{§8. Quantized Logarithmic Contact Manifolds}

We remember that a ringed space over $C$ is a pair $(X, \mathcal{A})$ where $X$ is a topological space and $\mathcal{A}$ a sheaf of $\boldsymbol{C}$-Algebras on $X$. Usually we will omit the 
expression "over $C$ ".

Given ringed spaces $(X, \mathcal{A})$ and $(Y, \mathscr{B})$ a morphism of ringed spaces from $(X, \mathcal{A})$ into $(Y, \mathscr{B})$ is a pair $(\varphi, \Phi)$ where $\varphi$ is a continuous map from $X$ into $Y$ and $\Phi$ is a morphism of $\mathbb{C}$-Algebras from $\varphi^{-1} \mathscr{B}$ into $\mathcal{A}$.

Definition 8.1. Let $(X, A)$ be a ringed space. We call an adjoint morphism of $(X, \mathcal{A})$ to an anti isomorphism $(a, *):(X, \mathcal{A}) \rightarrow\left(X, a^{-1} \mathcal{A}\right)$ such that $a^{-1} \mathcal{A}$ is isomorphic to $\mathcal{A}$ and $(a, *)^{2}=\operatorname{id} \mathcal{A}$.

We will in general write $\mathscr{A}^{a}$ and $\mathscr{A}^{*}$ instead of $a^{-1} \mathscr{A}$ and $*(\mathcal{A})$.

We call a ringed space with an adjoint morphism a self dual ringed space. We say that a subsheaf $\mathscr{B}$ of a self dual ringed space is self dual if $\mathscr{B}^{*}=\mathscr{B}$. We say that a morphism of ringed spaces between two self dual ringed spaces is self dual if it commutes with the adjoint morphisms.

Example 8.2. (i) Let $X_{n}$ be a copy of $\mathbb{C}^{n}$ with coordinates $\left(x_{1}, \cdots, x_{n}\right)$. Given an open set $U$ of $T^{*} X_{n}$ and a total symbol $P=\sum_{k} P_{k} \in \mathcal{E}_{X_{n}}(U)$ we denote by $P^{*}$ the total symbol $\sum_{k} Q_{k} \in \mathcal{E}_{X_{n}}^{a}(U)$, where

$$
Q_{l}(x,-\xi)=\sum_{\substack{l=k-|\alpha| \\ \alpha \in N}} \frac{(-)^{|\alpha|}}{\alpha !} \partial_{\xi}^{\alpha} \partial_{x}^{\alpha} Q_{k} .
$$

We call $*$ the adjoint morphism of $\mathcal{E}_{X_{n}}$. The pair $(a, *)$ is an adjoint morphism.

(ii) There is one and only one morphism of filtered $\mathbb{C}$-Algebras

$$
*: \varepsilon_{\left\langle X_{n} / Y_{\nu}\right\rangle} \rightarrow \mathcal{E}_{\left\langle X_{n} / Y_{y}\right\rangle}^{a}
$$

such that the following diagram commutes.

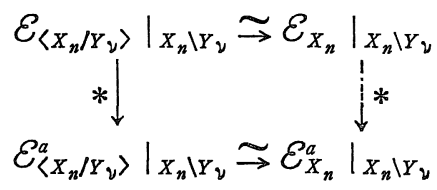

This morphism is an adjoint morphism.

For the proof of (i) cf. [SKK]. The existence of the morphism introduced in (ii) is a straightforward consequence of Theorem 5.10 and Corollary 5.15

Proposition 8.3. A quantized contact transformation

$$
\Phi:\left.\varphi^{-1} \mathcal{E}_{\left\langle X_{n} / Y_{y}\right\rangle} \rightarrow \mathcal{E}_{\langle X / Y\rangle}\right|_{U}
$$

is self dual iff 


$$
\begin{gathered}
\Phi\left(x_{i}\right)^{*}=\Phi\left(x_{i}\right), \quad 1 \leq i \leq n, \\
\Phi\left(\delta_{x_{i}}\right)+\Phi\left(\delta_{x_{i}}\right)^{*}= \begin{cases}1 & \text { if } 1 \leq i \leq \nu \\
0 & \text { if } \nu+1 \leq i \leq n\end{cases}
\end{gathered}
$$

Proof. It is a straightforward consequence of Theorem 5.10 and Corollary 5.15 .

Q.E.D.

Definition 8.4. A [self dual] quantized logarithmic contact manifold is given by the data (i), (ii), (iii) verifying the condition (iv).

(i) A filtered [self dual] ringed space $(X, \mathcal{E})$ where $X$ is an homogeneous logarithmic symplectic manifold $X$.

(ii) An open covering $U_{i}, i \in 1$, of $X$ by conic open sets and homogeneous canonical transformations $\varphi_{i}: U_{i} \rightarrow T^{*}\left\langle X_{n} / Y_{v_{i}}\right\rangle$

(iii) Isomorphisms of [self dual] filtered $\mathbb{C}$-Algebras

$$
\Phi_{i}:\left.\varphi_{i}^{-1} \mathcal{E}_{\left\langle X_{n} / Y_{\nu_{i}}\right\rangle} \rightarrow \mathcal{E}\right|_{U_{i}} .
$$

(iv) The isomorphisms of filtered $\boldsymbol{C}$-Algebras

$$
\Phi_{j}^{-1} \Phi_{i}:\left.\varphi_{i j}^{-1} \mathcal{E}_{\left\langle x_{n} / Y_{v_{i}}\right\rangle} \rightarrow \mathcal{E}_{\left\langle x_{n} / Y_{v_{j}}\right\rangle}\right|_{V_{i j}} .
$$

is a quantization of $\varphi_{i j}$. Here $V_{i j}$ equals $\varphi_{j}\left(U_{i} \cap U_{j}\right)$ and $\varphi_{i j}$ equals $\varphi_{i} \varphi_{j}^{-1}: V_{i j}$ $\rightarrow T^{*}\left\langle X_{n} / Y_{\nu_{j}}\right\rangle$.

Remark 8.5. (i) In general we will speak of the quantized contact manifold $(X, \mathcal{E})$, omiting the other data. If the homogeneous logarithmic contact manifold $X$ has poles along a divisor $Y$ we say that $(X, \mathcal{E})$ has poles along $Y$.

(ii) If $X$ is a homogeneous symplectic manifold we say that $(X, \mathcal{E})$ is a [self dual] quantized contact manifold.

(iii) There are obvious generalizations of the notions of [self dual] quantized contact transformation and quantized contact coordinates to the context of [self dual] quantized logarithmic contact manifolds.

(iv) We understand a [self dual] quantized logarithmic contact manifold as a pair $(X, \mathcal{E})$ where $X$ is the patching of a family of copies $U_{i}$ of homogeneous open sets of logarithmic cotangent bundles $T^{*}\left\langle X_{n} / Y_{v_{i}}\right\rangle$ by homogeneous canonical transformations and $\mathcal{E}$ is the glueing of the sheaves $\varepsilon_{\left\langle X_{n} / Y_{\nu_{i}}\right\rangle}$ along [self dual] quantizations of the homogeneous canonical transformations refered above.

Example 8.6. (i) Let $X$ be a complex manifold and $Y$ a divisor with normal crossings of $Y$. Then 


$$
\mathcal{E}_{\langle X / Y\rangle} \mid \dot{\circ}^{*}\langle X / Y\rangle
$$

is a quantized contact manifold. In general it is not self dual.

(ii) Put $\omega_{X}=\Omega^{\text {dimX } X}$. Let $\mathcal{L}_{1}, \mathcal{L}_{2}$ be invertible $\mathcal{O}_{X}$-modules defined in an open set of $X$. If $\varphi_{1}, \varphi_{2}: \mathcal{L}_{1} \rightarrow \mathcal{L}_{2}$ are isomorphisms such that $\varphi_{i}(s)^{\otimes 2}=s^{\otimes 2}$ for any local section $s$ of $\mathcal{L}_{1}$ then $\varphi_{1}= \pm \varphi_{2}$. Locally there is allways an isomorphism $\varphi: \mathcal{L}_{1} \rightarrow \mathcal{L}_{2}$. Moreover locally there is always an invertible $\mathcal{O}_{X}$-module $\mathcal{L}_{U}$ such that $\stackrel{\mathcal{L}^{\otimes 2}}{\rightarrow} \omega_{X}$. Therefore we can glue in a canonical way the sheaves

$$
\left.\mathcal{L}_{U} \otimes \mathcal{E}_{\langle X / Y\rangle}\right|_{U} \otimes \mathcal{L}_{U}^{\otimes(-1)},
$$

where $\mathcal{L}_{U}$ is a locally free $\mathcal{O}_{X}$-module defined in an open set $U$ of $T^{*}\langle X / Y\rangle$ such that $\mathcal{L}_{U}^{\otimes 2}$ is isomorphic to $\left.\omega_{X}\right|_{U}$, into a sheaf on $T^{*}\langle X / Y\rangle$ that we will denote by

$$
\omega_{X}^{\otimes 1 / 2} \otimes \mathcal{E}_{\langle X / Y\rangle} \otimes \omega_{X}^{\otimes-1 / 2}
$$

The adjoint morphism introduced in 8.2 (ii) induces an adjoint morphism in (8.9.1) and the restriction of (8.9.1) to $\stackrel{\circ}{*}^{*}\langle X \mid Y\rangle$ has a canonical structure of self dual quantized contact manifold with poles along $\AA^{-1}(Y)$.

Definition 8.7. Given a self dual quantized logarithmic contact manifold $(X, \mathcal{E})$, a connected open set $U$ of $X$ and $P \in \mathcal{E}_{(m)}(U)$ we define

$$
\sigma_{m}^{\prime}(P)=\frac{1}{2} \sigma_{m-1}\left(P-(-)^{m} P^{*}\right) .
$$

We call $\sigma_{m}^{\prime}(P)$ the subprincipal symbol of $P$ of order $m$.

We define $\sigma^{\prime}(P)$ as $\sigma_{m}^{\prime}(P)$ if $P$ has order $m \in \mathbb{Z}$ and zero if $P$ equal zero. We call $\sigma^{\prime}(P)$ the subprincipal symbol of $P$.

Proposition 8.8. (i) If $P \in \mathcal{E}_{(m-1)}$ then $\sigma_{m}^{\prime}(P)$ equals $\sigma_{m-1}(P)$.

(ii) If $P \in \mathcal{E}_{(m)}, Q \in \mathcal{E}_{(n)}$, then

$$
\sigma_{m+n}^{\prime}(P Q)=\sigma_{m}(P) \sigma_{n}^{\prime}(Q)+\sigma_{m}^{\prime}(P) \sigma_{n}(Q)+\frac{1}{2}\left\{\sigma_{m}(P), \sigma_{n}(Q)\right\}
$$

(iii) Let $\left(x_{1}, \cdots, x_{n}\right)$ be a system of coordinates on a open set $U$ of a complex manifold $X$ and let $(x, \xi)$ be the associated system of canonical coordinates of $T^{*} X$. Let $V$ be an open set of $\pi^{-1}(U)$ and $P$ a section of $\mathcal{E}_{X}(m)(V)$.

Then $\sigma_{m}^{\prime}\left(d x^{\otimes 1 / 2} \otimes P \otimes d x^{\otimes(-1 / 2)}\right)$ equals

$$
d x^{\otimes 1 / 2} \otimes\left(P_{m-1}-\sum_{i=1}^{n} \frac{\partial^{2} P_{m}}{\partial \xi_{i} \partial x_{i}}\right) \otimes d x^{\otimes(-1 / 2)}
$$


Proposition 8.9. Let $(X, \mathcal{E})$ be a self dual quantized contact manifold with poles along a smooth divisor $Y$. Let $Z$ be the residual submanifold of $X$.

(i) There is one and only one both side Ideal $\mathcal{G}_{Y}$ of $\mathcal{E}$ that is prime along the set of poles of $X$.

(ii) For each $\lambda \in C$ there is one and only one both side Ideal $\mathcal{G}_{\lambda}$ that is maximal along $Z$ and moreover is contained in the set of the local sections $P$ of $\mathcal{E}$ such that

$$
\xi \sigma^{\prime}(P) \equiv \lambda \sigma(P) \quad\left(\bmod I_{Y}+I_{Z}^{2}\right) .
$$

Here $\xi$ denotes an arbitrary residual function.

The ideal $\mathcal{G}_{\lambda}$ is self dual iff $\lambda=0$.

The sheaf of $\boldsymbol{C}$-Algebras $\mathcal{E} / \mathcal{J}_{0}$ is a self dual quantization of the contact manifold $Z$.

Definition 8.10. (i) We call $\mathcal{G}_{Y}$ the ideal of the set of poles of $(X, \mathcal{E})$.

(ii) We call $\mathcal{G}_{0}$ the self dual residual ideal.

Proof of Proposition 8.9. Statement (i) is a straightforward consequence of Theorem 6.6. It is enough to prove statement (ii) locally. By Theorem 6.6 it is enough to show that $\left(\delta_{x_{1}}+\lambda+\frac{1}{2}\right)$ is contained in

$$
\left\{P \in \mathcal{E}_{\left\langle X n / Y_{1}\right\rangle}: \xi_{1} \sigma^{\prime}(P) \equiv \lambda_{0} \sigma(P)\left(\bmod \left(x_{1}\right)+\left(\xi_{1}^{2}\right)\right)\right\}
$$

iff $\lambda=\lambda_{0}$.

By Remark 6.5 if $P \in\left(\delta_{x_{1}}+\lambda+\frac{1}{2}\right)$ then there are $R, S \in \mathcal{E}_{\left\langle x_{n} / Y_{1}\right\rangle}$ such that $P=S\left(\delta_{x_{1}}+\lambda+\frac{1}{2}\right)+x_{1} R$. Now, by Proposition 8.8 (ii),

$$
\begin{aligned}
\sigma^{\prime}(P) & \equiv \sigma^{\prime}(S) \xi_{1}+\lambda \sigma(S) & & \left(\bmod \left(x_{1}\right)\right) \\
\xi_{1} \sigma^{\prime}(P) & \equiv \lambda \xi_{1} \sigma(S) & & \left(\bmod \left(x_{1}\right)+\left(\xi_{1}^{2}\right)\right) \\
& \equiv \lambda \sigma(P) & & \left(\bmod \left(x_{1}\right)+\left(\xi_{1}^{2}\right)\right)
\end{aligned}
$$

Moreover $\xi_{1} \sigma^{\prime}\left(\delta_{x_{1}}+\lambda+\frac{1}{2}\right)=\lambda \sigma\left(\delta_{x_{1}}\right)$ and $\left(\lambda-\lambda_{0}\right) \xi_{1} \equiv 0\left(\bmod \left(x_{1}\right)+\left(\xi_{1}^{2}\right)\right)$ iff $\lambda=\lambda_{0}$.

Q.E.D.

\section{Chapter III. Blow up of a Holonomic System}

In $\S 9$ we show that the blow up $\pi: \widetilde{X} \rightarrow X$ of a contact manifold $X$ along a closed Lagrangian submanifold $\Lambda$ has a canonical structure of logarithmic contact manifold with poles along the exceptional divisor of $\tilde{X}$. We also show that, given a quantization $\mathcal{E}$ of $X$ there is a canonical quantization $\tilde{\mathcal{E}}$ of $\tilde{X}$ and a canonical morphism $\Phi: \pi^{-1} \mathcal{E}_{\Lambda} \rightarrow \tilde{\mathcal{E}}$, where $\mathcal{E}_{\Lambda}$ is a well known subsheaf of $\mathcal{E}$. 
Let $X$ be a logarithmic contact manifold with poles along a smooth divisor. In $\S 10$ we study the blow up $\pi: \tilde{X} \rightarrow X$ of $X$ along its residual submanifold. We show that $\tilde{X}$ has a canonical structure of logarithmic contact manifold. We also show how to associate to a quantization of $X$ a quantization of $\tilde{X}$.

Proposition 11.13 relates the construction presented in this Chapter with the construction described in the Introduction. Let $X$ be a complex manifold and $\lambda$ a point of $X$. Let $\pi_{X}: \mathbb{P}^{*} X \rightarrow X$ be the projective cotangent bundle of $X$. Put $\Lambda=\pi^{-1}(\lambda)$. Let $X_{0}$ be the blow up of $\mathbb{P}^{*} X$ along $\Lambda$ and let $X_{1}$ be the blow up of $X_{0}$ along its residual set. Let $Y_{1}$ be the set of poles of $X_{1}$. Then $X_{1} \backslash Y_{1}$ is a contact manifold isomorphic to $\mathbb{P}^{*} \widetilde{X} \backslash \mathbb{P}_{E}^{*} \tilde{X}$, where $\tilde{X}$ is the blow up of $X$ along $\{\lambda\}$ and $E$ the exceptional divisor of $\tilde{X}$.

Nevertheless we do not define the "total blow up of $\mathbb{P}^{*} X$ along $\Lambda^{\prime \prime}$ as $X_{1} / Y_{1}$. There is a loss of information when we take away the set $Y_{1}$. To minimize it we define recursively a family $\left(X_{k}\right)$ of logarithmic contact manifolds by putting $X_{k+1}=$ "blow up of $X_{k}$ along its residual submanifold". We put $Y_{k}=$ "set of poles of $X_{k}$ ". Finally we define the total blow up $\mathbb{P}^{*} X$ of $\mathbb{P}^{*} X$ as the union of the family of contact manifolds $\left(X_{k} \backslash Y_{k}\right)$. Here we identify $X_{k} \backslash Y_{k}$ with a canonical open set of $X_{k+1} \backslash Y_{k+1}$.

We show that $\mathbb{P}^{*} X$ has a canonical quantization. Finally we show how to associate to a holonomic $\mathcal{E}_{X}$-module $\mathscr{M}$ a holonomic $\tilde{\mathcal{E}}$-module $\tilde{\mathscr{M}}$.

\section{§9. Blow up of a Quantized Contact Mamifold along a Lagramgian Submanifold}

9.1. Given a scheme $S$ of finite type over $\mathbb{C}$ we will still denote by $S$ the associated analytic space. Given a nonnegative integer $n$ we will call the analytic space $\operatorname{Spec}\left(\mathbb{C}\left[x_{1}, \cdots, x_{n}\right]\right)$ a affine complex manifold of dimension $n$.

Definition 9.1.1. An affine logarithmic contact manifold $\mathbb{X}$ of dimension $2 n+1$ is given by the following data:

(i) A polynomial algebra $A$ over $\mathbb{C}$.

(ii) An algebraic basis $x_{1}, \cdots, x_{2 n+1}$ of $A$.

(iii) A subset $\varpi$ of $[1, n+1]$.

If $\varpi=\phi$ we call $X$ an affine contact manifold.

Put $X=\operatorname{Spec} A$ and $Y=\left\{\mathbb{I} i \in \varpi x_{i}=0\right\}$. The set $Y$ is a divisor with normal crossings of $X$. We call 


$$
\omega=\delta_{x_{n+1}}-\sum_{i=1}^{n} x_{i+n+1} \delta_{x_{i}}
$$

the canonical logarithmic contact form of $X$.

For instance, if $\varpi=\{1,2, n+1\}$, then

$$
\omega=\frac{d x_{n+1}}{x_{n+1}}-x_{n+2} \frac{d x_{1}}{x_{1}}-x_{n+3} \frac{d x_{2}}{x_{2}}-\sum_{i=3}^{n} x_{n+1+i} d x_{i} .
$$

We will in general denote the affine logarithmic contact manifold $\boldsymbol{X}$ simply by

$$
\left[\delta x_{n+1}-\sum_{i=1}^{n} x_{i+n+1} \delta x_{i}\right]
$$

Given (9.1.1) we recover the data (i), (ii), (iii) in the following way. We put $A=C\left[x_{1}, \cdots, x_{2 n+1}\right]$ and we choose for $\varpi$ the smallest subset $I$ of $[1, n+1]$ such that $\left(\prod_{i \in I} x_{i}\right) \omega$ is a differential form on $X$.

Definition 9.1.2. An affine homogeneous logarithmic symplectic manifold $\boldsymbol{X}$ of dimension $2 n$ is given by the following data:

(i) A polynomial algebra $A$ over $\boldsymbol{C}$ with a structure of graded ring.

(ii) An algebraic basis $\left(x_{1}, \cdots, x_{n}, \xi_{1}, \cdots, \xi_{n}\right)$ of $A$ such that $x_{1}, \cdots, x_{n}$ are homogeneous of degree 0 and $\xi_{1}, \cdots, \xi_{n}$ are homogeneous of degree 1 .

(iii) Subsets $\varpi, \iota$ of $[1, n]$, where $\iota$ is nonempty.

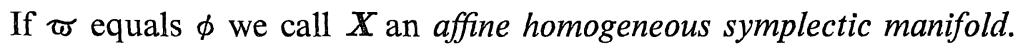

Put $X=\operatorname{Spec} A \backslash \cap_{i \in \iota}\left\{\xi_{i}=0\right\}$ and $Y=\left\{\prod_{i \in \varpi} x_{i}=0\right\} \cap X$. We call $\theta=$ $\sum \xi_{i} \delta x_{i}$ the canonical 1-form of $\boldsymbol{X}$. We will in general denote the affine logarithmic contact manifold $\boldsymbol{X}$ simply by

$$
\left[\sum_{i=1}^{n} \xi_{i} \delta x_{i}\right]_{i} .
$$

If $\iota=\{k\}$ then we denote $\boldsymbol{X}$ by $\left[\sum_{i=1}^{n} \xi_{i} \delta x_{i}\right]_{k}$. If $\iota=[1, n]$ then we denote $\boldsymbol{X}$ by $\left[\sum_{i=1}^{n} \xi_{i} \delta x_{i}\right]$.

Defimition 9.1.4. Given an affine logarithmic contact manifold $\boldsymbol{X}$ of dimension $2 n+1$ we associate to it the affine homogeneous logarithmic symplectic manifold $\hat{\boldsymbol{X}}$ given by the following data.

(i) The $\boldsymbol{C}$-algebra $A \otimes_{\boldsymbol{C}} \boldsymbol{C}\left[\omega^{\otimes(-1)}\right]$, where $\omega$ is the canonical contact form of $\boldsymbol{X}$. We endow $A \otimes_{C} \boldsymbol{C}\left[\omega^{\otimes(-1)}\right]$ with the only graduation such that all the elements of $A$ have degree 0 and $\omega^{\otimes(-1)}$ has degree 1 .

(ii) The algebraic basis $x_{1}, \cdots, x_{n+1}, \xi_{1}, \cdots, \xi_{n+1}$, where

$$
\xi_{i}=-p_{i} \omega^{\otimes(-1)}, \quad 1 \leq i \leq n, \quad \xi_{n+1}=\omega^{\otimes(-1)} .
$$


(iii) The sets $\approx$ and $\{n+1\}$.

We call $\hat{X}=\left[\sum_{i=1}^{n+1} \xi_{i} d x_{i}\right]_{n+1}$ the affine homogeneous logarithmic symplectic manifold associated to the affine logarithmic contact manifold $\mathbb{X}$.

Remark 9.1.5. Given an affine homogeneous symplectic manifold $\mathbb{X}$ of dimension $2 n$ let $M$ be the Spectrum of the subalgebra of $A$ generated by $x_{1}, \cdots, x_{n}$. Put $N=\left\{\mathbb{I}_{\mathrm{i} \in \varpi} x_{i}=0\right\} \subset M$. We identify $\mathbb{T}^{*}\langle M \mid N\rangle$ with Spec $A$. Let $\iota$ be the open inclusion $X \hookrightarrow T^{*}\langle M \mid N\rangle$. We call the sheaf of $\mathbb{C}$-algebras $\mathcal{E}=\iota^{-1} \mathcal{E}_{\langle M / N\rangle}$ the quantization of the affine homogeneous logarithmic symplectic manifold $X$.

Let $(X, \mathcal{L})$ be a contact manifold. Let $\Lambda$ be a closed Lagrangian submanifold of $X$ and $I_{\Lambda}$ the defining ideal of $\Lambda$. Let

$$
\pi: \tilde{X}=\operatorname{Projan}\left(\oplus_{k \geq 0} I_{A}^{k}\right) \rightarrow X
$$

be the blow up of $X$ along $\Lambda$ ). Let $E$ be the exceptional divisor of $\pi$ and $I_{E}$ its defining ideal. We can identify the dual $\mathcal{O}_{(E)}$ of $I_{E}$ in a canonical way with a subsheaf of $j_{*} \mathcal{O}_{\tilde{X} \backslash E}$, where $j: \widetilde{X} \backslash E \hookrightarrow \widetilde{X}$ is the inclusion map.

Proposition 9.2. The $\mathcal{O}_{\tilde{X}}$-module $\tilde{\mathcal{L}}=\mathcal{O}_{(E)} \pi^{*} \mathcal{L}$ is a structure of logarithmic contact manifold with poles along $E$. It is the only structure of logarithmic contact manifold on $\tilde{X}$ such that the restriction of $\pi$ to $\tilde{X} \backslash E$ is a contact transformation.

Defimition 9.3. We call the pair $(\pi: \tilde{X} \rightarrow X, \tilde{\mathcal{L}})$ blow up of the contact manifold $(X, \mathcal{L})$ along its (closed) Lagrangian submanifold $\Lambda$.

Proposition 9.40. Let $X$ be a complex manifold. Let $\lambda$ be a point of $X$ and put $\Lambda=\pi_{X}^{-1}(\lambda) \subset \mathbb{P}^{*} X$. Then the blow up of $\mathbb{P}^{*} X$ along $\Lambda$ equals the logarithmic contact manifold $\mathbb{P}^{*}\langle\tilde{X} \mid E\rangle$, where $\tilde{X}$ is the blow up of $X$ along $\{\lambda\}$ and $E$ is the exceptional divisor of $\tilde{X} \rightarrow X$.

Proof of Proposition 9.2. Since the problem is local in $X$ we can suppose that $X$ is the affine logarithmic contact manifold $\left[d x_{n+1}-\sum_{i=1}^{n} p_{i} d x_{b}\right]$ and that $\Lambda=\left\{x_{1}=\cdots=x_{n+1}=0\right\}$. Put $z=x_{n+1}-\sum_{i=1}^{n} x_{i} p_{i}$. Then $\tilde{X}$ is the obvious patching of the affine complex manifolds.

$$
\begin{aligned}
X_{k} & =\operatorname{Spec}\left(\mathbb{C}\left[x_{k}, \frac{x_{j}}{x_{k}}, j \neq k, \frac{z}{x_{k}}, p_{j}, j \neq k\right]\right), \quad 1 \leq k \leq n, \\
X_{n+1} & =\operatorname{Spec}\left(\mathbb{C}\left[z, \frac{x_{j}}{z}, 1 \leq j \leq n, p_{j}, 1 \leq j \leq n\right]\right) .
\end{aligned}
$$


Moreover $E \cap X_{k}=\left\{x_{k}=0\right\}, 1 \leq k \leq n, E \cap X_{n+1}=\{z=0\}$. Put $\omega_{k}=\left.\pi^{*} \omega\right|_{X_{k}}$, $1 \leq k \leq n+1$. Then the $\mathcal{O}_{X}$-module $\mathcal{L}$ is determined by the logarithmic differential forms

$$
\begin{aligned}
& \frac{\omega_{k}}{x_{k}}=d \frac{x_{n+1}}{x_{k}}-\frac{z}{x_{k}} \frac{d x_{k}}{x_{k}}-\sum_{j \neq k} p_{j} d x_{j}, \quad \text { if } 1 \leq k \leq n \\
& \frac{\omega_{n+1}}{z}=\frac{d z}{z}+\sum_{j=1}^{n} \frac{x_{j}}{z} d p_{j},
\end{aligned}
$$

Proof of Proposition 9.4. There is a canonical open immersion

$$
\left.\boldsymbol{P}^{*}\langle\tilde{X} / E\rangle\right|_{\tilde{X} \backslash E} \rightarrow \boldsymbol{P}^{\tilde{*}} X .
$$

Here $\boldsymbol{P}^{*}\langle\tilde{X} \mid E\rangle$ denotes the blow up of $\boldsymbol{P}^{*} X$ along $\Lambda$.

Since $\boldsymbol{P}^{*}\langle\tilde{X} \mid E\rangle|\tilde{X}| E$ is dense in $\boldsymbol{P}^{*}\langle\tilde{X} \mid E\rangle$ then its enough to show that (9.4.1) admits locally an extension to $\boldsymbol{P}^{*}\langle\tilde{X} \mid E\rangle$. This can be easily shown by some simple computations with local coordinates.

Q.E.D.

Let $(X, \mathcal{E})$ be a quantized contact manifold and $A$ a (closed) Lagrangian submanifold of $X$. Let $I_{A}$ be the defining ideal of $\Lambda, \mathcal{I}_{A}$ the sub $\mathcal{E}_{(0) \text {-module }}$ of $\mathcal{E}_{(1)}$ of the microdifferential operators $P \in \mathcal{E}_{(1)}$ such that $\sigma_{1}(P) \in I_{\Lambda}$. Following Kashiwara-Oshima [13] we define

$$
\mathcal{E}_{\Lambda}=\sum_{k \geq 1} \mathcal{J}_{\Lambda}^{k}
$$

The $\boldsymbol{C}$-algebra $\mathcal{E}_{\Lambda}$ is noetherian and has zariskian fibers. Moreover if $(X, \mathcal{E})$ is self dual then $\mathcal{E}_{\Lambda}$ is also self dual.

Theorem 9.5. Let $(X, \mathcal{E})$ be a [self dual] quantized contact manifold and $A$ a closed Lagrangian submanifold of $X$. Then there is a [self dual] quantization $\tilde{\mathcal{E}}$ of the blow up of the contact manifold $X$ along $\Lambda$ and $a$ [self dual] morphism

$$
\Phi: \pi^{-1} \mathcal{E}_{\Lambda} \rightarrow \tilde{\mathcal{E}}
$$

such that $\left.\Phi\right|_{\tilde{X} \backslash E}:\left.\left.\pi^{-1} \mathcal{E}_{\Lambda}\right|_{\tilde{X} \backslash E} \rightarrow \tilde{\mathcal{E}}\right|_{\tilde{X} \backslash E}$ is an isomorphism. Moreover the pair $(\Phi, \tilde{\mathcal{E}})$ is unique up to a unique isomorphism.

Definition 9.6. The pair $\left(\pi: \tilde{X} \rightarrow X, \Phi: \pi^{-1} \mathcal{E}_{\Lambda} \rightarrow \tilde{\mathcal{E}}\right)$ is called the blow up of the [self dual] quantized contact manifold $(X, \mathcal{E})$ along the Lagrangian submanifold $\Lambda$.

Lemma 9.7. There is one and only one morphism of $\pi^{-1} \mathcal{O}_{X}$-modules

$$
\varphi: \pi^{-1} \operatorname{gr} \mathcal{E}_{\Lambda} \rightarrow \oplus_{k \in \boldsymbol{Z}} \tilde{\mathcal{L}}^{\otimes(-k)}
$$


that extends the natural isomorphism

$$
\left.\left.\pi^{-1} \operatorname{gr} \mathcal{E}\right|_{\tilde{X} \backslash E} \rightarrow \bigoplus_{k \in Z} \tilde{\mathcal{L}}^{\otimes(-k)}\right|_{\tilde{X} \backslash E}
$$

induced by $\pi$.

Remark 9.8. The sheaf of graded algebras gr $\tilde{\mathcal{E}}$ equals $\oplus_{k \in Z} \tilde{\mathcal{L}}^{\otimes(-k)}$. Moreover the morphism

$$
\operatorname{gr} \Phi: \pi^{-1} \text { gr } \varepsilon_{\Lambda} \rightarrow \operatorname{gr} \tilde{\varepsilon}
$$

equals the morphism (9.7.1).

Proof of the Lemma 9.7. Since $\tilde{X} \backslash E$ is dense in $\tilde{X}$ it is enough to prove the existence of $\varphi$ locally on $X$. We will use the notations and assumptions of the proof of Proposition 9.3. We notice that

$$
\operatorname{gr} \mathcal{E}_{\Lambda}=\oplus_{k \in Z} I_{\Lambda}^{k} \otimes \mathcal{L}^{\otimes(-k)},
$$

where $I_{\Lambda}^{k}$ equals $\mathcal{O}_{X}$ if $k \leq 0$. Given an open set $U$ of $X, f=\left(f_{1}, \cdots, f_{n_{1}}\right) \in \mathcal{O}(U)^{n+1}$, $g \in \mathcal{O}(U)$ put $\frac{f}{g}=\left(\frac{f_{1}}{g}, \cdots, \frac{f_{n+1}}{g}\right)$. Put $I_{k}=\left\{\alpha \in N^{n+1}:|\alpha|=k\right\}$. The free $\mathcal{O}_{X}$-module $I_{\Lambda}^{k} \otimes \mathcal{L}^{\otimes(-1)}$ admits the basis

$$
x^{\alpha} \otimes \omega^{\otimes(-k)}, \quad \alpha \in I_{k},
$$

and

$$
z^{b} x^{\beta} \otimes \omega^{\otimes(-k)}, \quad(b, \beta) \in I_{k}
$$

Since

$$
\left.\pi^{*}\left(x^{\alpha} \otimes \omega^{\otimes(-k)}\right)\right|_{x_{i}}=\left(\frac{x}{x_{i}}\right)^{\alpha} \omega_{i}^{\otimes(-k)}, \quad 1 \leq i \leq n
$$

and

$$
\left.\pi^{*}\left(z^{b} x^{\beta}\right) \otimes \omega^{\otimes(-k)}\right)\left.\right|_{x_{n+1}}=\left(\frac{x}{z}\right)^{\beta} \otimes \omega_{n+1}^{\otimes(-k)}
$$

then we can built morphisms

$$
\varphi_{i}:\left.\left.\pi^{-1} \operatorname{gr} \varepsilon_{\Lambda}\right|_{X_{i}} \rightarrow \oplus_{k} \tilde{\mathcal{L}}^{\otimes(-k)}\right|_{X_{i}}, \quad 1 \leq i \leq n+1,
$$

that extend the natural isomorphisms

$$
\left.\left.\pi^{-1} \operatorname{gr} \mathcal{E}\right|_{X_{i} \backslash E} \rightarrow \bigoplus_{k} \tilde{\mathcal{L}}^{\otimes(-k)}\right|_{X_{j} \backslash E}, \quad 1 \leq i \leq n+1 .
$$

We can glue the morphisms $\varphi_{i}$ into the desired morphism $\varphi$.

Q.E.D。

We will now prove the Theorem in the case $X$ is the quantization of the affine contact manifold $\left[d x_{n+1}-\sum_{i=1}^{n} p_{i} d x_{i}\right]$. We will use this assumption in para- 
graphs $9.9,9.10$, and 9.11 .

9.9. We will now quantize the homogeneous logarithmic symplectic manifold $\hat{\tilde{X}}$. We know from the proof of Proposition 9.2 that $\tilde{X}$ is the patching of the affine logarithmic contact manifolds $X_{k}, 1 \leq k \leq n+1$, where

$$
\begin{aligned}
X_{k} & =\left[d \frac{x_{n+1}}{x_{k}}+\frac{z}{x_{k}} \frac{d x_{k}}{x_{k}}-\sum_{j \neq k} p_{j} d \frac{x_{j}}{x_{k}}\right], \quad 1 \leq k \leq n, \\
X_{n+1} & =\left[\frac{d z}{z}+\sum_{i=1}^{n} \frac{x_{i}}{z} d p_{i}\right] .
\end{aligned}
$$

For $1 \leq k \leq n$ put

Put

$$
\begin{aligned}
& x_{k j}=\frac{x_{j}}{x_{k}}, \quad \xi_{k j}=x_{k} \xi_{j}, \quad j \neq k, \\
& x_{k k}=x_{k}, \quad \xi_{k k}=\sum_{i=1}^{n+1} x_{i} \xi_{i} . \\
& \begin{array}{rlrl}
x_{n+1, j} & =p_{j}, \quad \xi_{n+1, j} & =x_{j} \xi_{n+1}, & j \neq n+1, \\
x_{n+1, n+1} & =z, \quad \xi_{n+1, n+1} & =\sum_{i=1}^{n+1} x_{i} \xi_{i} .
\end{array}
\end{aligned}
$$

Now $\hat{\tilde{X}}$ is a patching of affine homogeneous logarithmic symplectic manifolds $\hat{X}_{k}, 1 \leq k \leq n+1$, where $\hat{X}_{k}$ equals

$$
\left[\xi_{k k} \frac{d x_{k k}}{x_{k k}}-\sum_{j \neq k} \xi_{k j} d x_{k j}\right]_{n+1},
$$

along the obvious contact transformations $\varphi_{k l}: \hat{X}_{k l} \rightarrow \hat{X}_{l}$. Here $X_{k l}=X_{k} \backslash\left\{x_{k l}=\right.$ $0\}$. For $1 \leq k \leq n+1$ let $\mathcal{E}_{k}$ be the quantization of $\hat{X}_{k}$. We glue the sheafs $\mathcal{E}_{k}$ by quantized contact transformations

$$
\Phi_{k l}:\left.\varphi_{l k}^{-1} \mathcal{E}_{k} \rightarrow \mathcal{E}_{l}\right|_{x_{l k}}
$$

defined as follows. If $1 \leq k, l \leq n, k \neq l$, then we define $\Phi_{k l}$ by

$$
\begin{array}{rlrl}
x_{k j} & \mapsto x_{l j} x_{l k}^{-1}, & \partial_{x_{k j}} \mapsto x_{l k} \partial_{x_{l j}}, & j \neq k, l, \\
x_{k k} \mapsto x_{l l} x_{l k}, & \delta_{x_{k k}} \mapsto \delta_{x_{l l}}, \\
x_{k l} \mapsto x_{l k}^{-1}, & \partial_{x_{k l}} \mapsto x_{l k}\left(\delta_{x_{l l}}-\sum_{i \neq l} x_{l i} \partial_{x_{l i}}-\frac{n}{2}\right) .
\end{array}
$$

If $k \neq n+1$ then we define $\Phi_{k, n+1}:\left.\varphi_{n+1, l}^{-1} \mathcal{E}_{k} \rightarrow \mathcal{E}_{n+1}\right|_{X_{n+1, k}}$ by

$$
\begin{aligned}
x_{k j} & \mapsto \partial_{x_{n+1}, j} \partial_{x_{n+1, k}}^{-1}, \\
\partial_{x_{k j}} & \mapsto-x_{n+1, j} \partial_{x_{n+1, k}}, \\
x_{k k} & \mapsto \partial_{x_{n+1, k} \delta_{x_{n+1, n+1}}^{-1}} x_{n+1, n+1} \\
\delta_{x_{k k}} & \mapsto \delta_{x_{n+1, n+1}},
\end{aligned}
$$




$$
\begin{aligned}
& x_{k, n+1} \mapsto \delta_{x_{n+1}, n+1} \partial_{x_{n+1, k}}^{-1}+\sum_{i=1}^{n} x_{n+1, i} \partial_{x_{n+1, i}} \partial_{x_{n+1, k}}^{-1}+\frac{n}{2} \partial_{x_{n+1, k}}^{-1} \\
& \partial_{x_{k, n+1}} \mapsto \partial_{x_{n+1, k}} .
\end{aligned}
$$

We obtain in this way a sheaf $\tilde{\mathcal{E}}$ on $\hat{\tilde{X}}$. Since the quantized contact transformations $\Phi_{k l}$ are self dual then $\tilde{\varepsilon}$ is a self dual quantization.

9.10. We will now build the morphism (9.5.1). We will first introduce self dual quantized contact transformations

$$
\Phi_{k}:\left.\left.\pi^{-1} \mathcal{E}\right|_{X_{k} \backslash\left\{x_{k k}=0\right\}} \rightarrow \mathcal{E}_{k}\right|_{X_{k} \backslash\left\{x_{k k}=0\right\}}, \quad 1 \leq k \leq n+1,
$$

in the following way. If $1 \leq k \leq n$ then we define $\Phi_{k}$ by

$$
\begin{array}{llll}
x_{j} \mapsto x_{k k} x_{k j}, & \partial_{x_{j}} \mapsto x_{k k}^{-1} \partial_{x_{k j}}, \quad j \neq k, \\
x_{k} \mapsto x_{k k} & \partial_{x_{k}} \mapsto x_{k k}^{-1}\left(\delta_{x_{k k}}-\sum_{i \neq k} x_{k i} \partial_{x_{k i}}-\frac{n}{2}\right) .
\end{array}
$$

We define $\Phi_{n+1}$ by

$$
\begin{aligned}
x_{j} & \mapsto \delta_{x_{n+1} n+1}^{-1} x_{n+1, n+1} \partial_{x_{n+1}, j}, \\
\partial_{x_{j}} & \mapsto-x_{n+1, n+1}^{-1} \delta_{x_{n+1, n+1}} x_{n+1, j}, \\
x_{n+1} & \mapsto x_{n+1, n+1}+\left(\sum_{i=1}^{n} x_{n+1, i} \partial_{x_{n+1, i}}+\frac{n}{2}\right) \delta_{x_{n+1, n+1}}^{-1} x_{n+1, n+1}, \\
\partial_{x_{n+1}} & \mapsto x_{n+1, n+1}^{-1} \delta_{x_{n+1, n+1}} .
\end{aligned}
$$

We will now extend $\Phi_{k}, 1 \leq k \leq n+1$, into a morphism from $\left.\pi^{-1} \mathcal{E}_{A}\right|_{x_{k}}$ into $\left.\tilde{\mathcal{E}}\right|_{x_{k}}$ that we will still denote by $\Phi_{k}$. Choose $p^{0} \in E \cap X_{k}$. Let $\tilde{\varepsilon}_{A}[\hat{\tilde{E}}]$ be the formal analog of $\varepsilon_{\Lambda}[\tilde{E}]$. Put $A=\hat{\varepsilon}_{\Lambda, p^{0}}$. Let $B$ be the subalgebra of $A$ of the formal microdifferential operators $P \in A$ such that there is a neighbourhood $\Omega$ of $p^{0}$ in $\tilde{X}$, a representative $P^{\prime}$ of $P$ in $\Omega$ and $Q \in \hat{\tilde{E}}(\Omega)$ such that $\Phi\left(\left.P^{\prime}\right|_{\Omega \backslash E}\right)=$ $\left.Q\right|_{\Omega \backslash E}$. We conclude from Theorem 5.10 and Corollary 5.15 that $B$ equals $A$. One can show, using the Cauchy estimates, that if $P \in \mathcal{E}_{1, p^{0}}$ then $Q \in \widetilde{\mathcal{E}}_{p^{0}}$. We can glue the $\Phi_{k}$ 's into the desired morphism $\Phi$. This morphism is self dual by construction.

Definition 9.10.3. We call the pair $\left(\pi: \tilde{X} \rightarrow X, \Phi: \pi^{-1} \mathcal{E} \rightarrow \tilde{\mathcal{E}}\right)$ the quantization of the blow up of the affine contact manifold $\left[d x_{n+1}-\sum_{i=1}^{n} p_{i} d x_{i}\right]$ along the Lagrangian submanifold $\left\{x_{1}=\cdots=x_{n+1}=0\right\}$.

9.11. Still under the assumption (9.8.1) let $\tilde{\mathcal{E}}^{\prime}$ be another [self dual] quantization of $\tilde{X}$ and $\Phi^{\prime}: \pi^{-1} \mathcal{E}_{\Lambda} \rightarrow \tilde{\mathcal{E}^{\prime}}$ another [self dual] morphism such that $\Phi^{\prime} \mid \tilde{X} \backslash E^{:}$ $\left.\pi^{-1} \mathcal{E}_{\Lambda} \rightarrow \tilde{\mathcal{E}}\right|_{\tilde{X} \backslash E}$ is an isomorphism. There is at most one morphism $\Psi: \tilde{E} \rightarrow \tilde{\mathcal{E}^{\prime}}$ such that $\Psi \circ \Phi=\Phi^{\prime}$. Put $\vartheta=\sum_{i=1}^{n+1} x_{i} \partial_{x_{i}}+\frac{n}{2}$. We notice that, for $1 \leq k \leq n$, 


$$
\begin{aligned}
\Phi_{k}\left(x_{k}\right) & =x_{k k}, & \Phi_{k}(\vartheta) & =\delta_{x_{k k}}, \\
\Phi_{k}\left(x_{k} \partial_{x_{j}}\right) & =\partial_{x_{k j}}, & \Phi_{k}\left(x_{j} \partial_{x_{j}}\right) & =x_{k i} \partial_{x_{k j}}, \\
\Phi_{k}\left(x_{j}\right) & =x_{k k} x_{k j}, & &
\end{aligned}
$$

Put $\Phi_{k}^{\prime}=\left.\Phi^{\prime}\right|_{x_{k}}$ for $1 \leq k \leq n+1$. Since $\sigma\left(\Phi_{k}^{\prime}(P)\right)=\sigma\left(\Phi_{k}(P)\right)$ for $1 \leq k \leq n+1$ and any $P$ then $\Phi_{k}^{\prime}\left(x_{k} \partial_{x_{j}}\right) \Phi_{k}^{\prime}\left(x_{j} \partial_{x_{j}}\right)^{-1}$ is defined outside $\left\{\xi_{k j}=0\right\}$ and $\Phi_{k}^{\prime}\left(x_{j}\right) \Phi^{\prime}\left(x_{k}\right)^{-1}$ is defined outside $\left\{x_{k k}=0\right\}$. Since this sections coincide in the intersections of their domains then there is one and only one extension of both to $X_{k}$ that we will denote by $\Phi_{k}^{\prime}\left(x_{j} / x_{k}\right)$. We define $\Psi_{k}:\left.\mathcal{E}_{k} \rightarrow \tilde{\mathcal{E}^{\prime}}\right|_{X_{k}}$ as the quantization of $\mathrm{id}_{X_{k}}$ determined by

$$
\begin{aligned}
x_{k k} & \mapsto \Phi_{k}^{\prime}\left(x_{k}\right), & \delta_{x_{k k}} & \mapsto \Phi_{k}^{\prime}(\vartheta), \\
x_{k j} & \mapsto \Phi_{k}^{\prime}\left(x_{j} / x_{k}\right), & \partial_{x_{k j}} \mapsto \Phi_{k}^{\prime}\left(x_{k} \partial_{x_{j}}\right), & j \neq k .
\end{aligned}
$$

We can build a morphism $\Psi_{n+1}:\left.\mathcal{E}_{n+1} \rightarrow \mathcal{E}^{\prime}\right|_{x_{n+1}}$ in a similar way. We define $\Psi$ as the glueing of the morphisms $\Psi_{k}, 1 \leq k \leq n+1$.

9.12. We will now finish the proof of Theorem 9.5.

Let $X_{\alpha}, \alpha \in I$, be an open covering of $\Lambda$ and for each $\alpha$ let $\iota_{\alpha}: X_{\alpha} \rightarrow \boldsymbol{X}=$ $\left[d x_{n+1}-\sum_{i=1}^{n} p_{i} d x_{i}\right]$ be quantized contact transformation such that $\iota_{\alpha}^{-1}\left\{x_{1}=\cdots\right.$ $\left.=x_{n+1}=0\right\}=\Lambda \cap X_{\alpha}$. Let $\mathcal{E}_{n+1}$ denote the quantization of the affine contact manifold $\boldsymbol{X}$ and put $\mathcal{E}_{\alpha}=\iota_{\alpha}^{-1} \mathcal{E}_{n+1}, X_{\alpha \beta}=X_{\alpha} \cap X_{\beta}, X_{\alpha}^{\prime}=X_{\alpha} \backslash \Lambda$.

We can understand the quantization $\mathcal{E}$ of $\boldsymbol{X}$ as the glueing of $\mathcal{E}_{\alpha}, \alpha \in I$, $\left.\mathcal{E}\right|_{X \backslash \Lambda}$ by quantized contact transformations

$$
\begin{aligned}
& \Phi_{\alpha \beta}:\left.\left.\mathcal{E}_{\alpha}\right|_{X_{\alpha \beta}} \rightarrow \mathcal{E}_{\beta}\right|_{X_{\alpha \beta}}, \\
& \Phi_{\alpha}^{\prime}:\left.\left.\mathcal{E}_{\alpha}\right|_{X_{\alpha}^{\prime}} \rightarrow \mathcal{E}\right|_{X_{\alpha}^{\prime}} .
\end{aligned}
$$

The contact transformation $\iota_{\alpha}$ induces a quantization

$$
\Phi_{\alpha}: \pi_{\alpha}^{-1} \mathcal{E}_{\alpha \Lambda_{\alpha}} \rightarrow \tilde{\mathcal{E}_{\alpha}}
$$

of the blow up of $X_{\alpha}$ along $\Lambda_{\alpha}$. If $X_{\alpha} \cap X_{\beta} \neq \phi$ then $\Phi_{\alpha \beta}$ induces a quantized contact transformation

$$
\tilde{\Phi}_{\alpha \beta}: \tilde{\mathcal{E}}_{\alpha}\left|\tilde{X}_{\alpha \beta} \rightarrow \tilde{\mathcal{E}}_{\beta}\right| \tilde{X}_{\alpha \beta} .
$$

If $\Phi_{\alpha \beta}$ is self dual then $\tilde{\Phi}_{\alpha \beta}$ is also self dual. The proof of the existence of $\Phi_{\alpha \beta}$ is quite similar to the reasoning of paragraph 9.9.

We can glue the morphisms of self dual filtered $\boldsymbol{C}$-Algebras $\Phi_{\alpha}$ into a morphism of [self dual] filtered $\boldsymbol{C}$-Algebras. 


$$
\Phi: \pi^{-1} \varepsilon_{\Lambda} \rightarrow \tilde{\varepsilon}
$$

Let now $\Phi^{\prime}: \pi^{-1} \mathcal{E}_{1} \rightarrow \tilde{\mathcal{E}^{\prime}}$ be another quantization of $\pi: \tilde{X} \rightarrow X$. The local uniqueness of a quantization $\Psi$ of the identity such that $\Psi \Phi=\Phi^{\prime}$ is obvious. Therefore it is enough to prove the existence locally. This is trivial outside the exceptional divisor. This was proven near the exceptional divisor in paragraph 9.11 .

This ends the proof of Theorem 9.5.

Proposition 9.13. The morphism $\Phi: \pi^{-1} \mathcal{E}_{\Lambda} \rightarrow \tilde{\mathcal{E}}$ is flat.

Proof. The morphism $\Phi$ is flat outside the exceptional divisor. Choose $p^{0} \in E$. We can suppose that $(X, \mathcal{E})$ is the quantization of the affine contact manifold $\left[d x_{n+1}-\sum_{i=1}^{n} p_{i} d x_{i}\right]$ and $x_{i}\left(\pi\left(p^{0}\right)\right)=p_{j}\left(\pi\left(p^{0}\right)\right)=0$ for $1 \leq i \leq n+1$, $1 \leq j \leq n$. Since the filtrations of $\mathcal{E}_{\Lambda, \pi\left(p^{0}\right)}$ and $\tilde{\mathcal{E}}_{p^{0}}$ are zariskian and the sequence

$$
0 \rightarrow \varepsilon_{\Lambda_{0} \pi\left(p^{0}\right)} \rightarrow \tilde{\varepsilon}_{p^{0}}
$$

is strictly exact it is enough to prove that the morphism

$$
\varphi_{p}{ }^{0}: \operatorname{gr} \varepsilon_{\Lambda_{0} \pi\left(p^{0}\right)} \rightarrow \operatorname{gr} \tilde{\varepsilon}_{p}^{0}
$$

is flat (cf. for instance Schapira [15]). Suppose that there is a $k, 1 \leq k \leq n$, such that $p^{0} \in X_{k}$. Put

$$
\begin{aligned}
& A_{k}=\boldsymbol{C}\left[x_{j} \eta_{n+1}, 1 \leq j \leq n+1, p_{j}, 1 \leq j \leq n, \eta_{n+1}^{-1}\right], \\
& B_{k}=\boldsymbol{C}\left[x_{k j}, 1 \leq j \leq n+1, p_{k j}, 1 \leq j \leq n, \eta_{k, n+1}, \eta_{k, n+1}^{-1}\right] .
\end{aligned}
$$

There are canonical immersions of $A_{k}\left[B_{k}\right]$ into gr $\mathcal{E}_{\Lambda, 0}\left[\mathrm{gr} \tilde{\varepsilon}_{p^{0}}\right]$. The morphism $\varphi_{p^{0}}$ induces morphisms $\varphi_{k}: A_{k} \rightarrow B_{k}$.

Let $I_{k}\left[\mathcal{G}_{k}\right]$ be the ideal of $A_{k}\left[\operatorname{gr} \mathcal{E}_{\Lambda, \pi\left(p^{0}\right)}\right]$ generated by $x_{1}, \cdots, x_{n+1}, p_{1}, \cdots, p_{n}$. Let $J_{k}\left[\mathcal{G}_{k}\right]$ be the ideal of $B_{k}\left[\operatorname{gr}{\tilde{\mathcal{E}_{p}}}^{0}\right]$ generated by $x_{k j}-x_{k j}\left(p^{0}\right), 1 \leq j \leq n+1$, $p_{k_{j}}-p_{k_{j}}\left(p^{0}\right), 1 \leq j \leq n$. The completion of $A_{k}\left[B_{k}\right]$ relative to the $I_{k}$-adic topology [ $J_{k}$-adic] topology equals the completion of $\operatorname{gr} \mathcal{E}_{\Lambda, \pi\left(p^{0}\right)}$ [gr $\tilde{\mathcal{E}}_{p^{0}}$ ] relative to the $\mathcal{G}_{k^{-}}$ adic topology [ $\mathscr{g}_{k}$-adic topology]. Since the maps $\varphi_{k}, \varphi_{p^{0}}$ are continuous and have the same completion relatively to the topologies introduced above it is enough to show that $\varphi_{k}$ is flat (cf. Bourbaky [1]). The morphism $\varphi_{k}$ is flat because $\varphi_{k}\left(x_{k} \eta_{n+1}\right)$ is invertible in $B_{k}$ and $\varphi_{k}$ induces an isomorphism

$$
A_{k_{x_{k} \eta_{n+1}}} \stackrel{\sim}{\rightarrow} B_{k}
$$

If $p^{0} \in X_{n+1}$ then we can prove the proposition in a similar way. Q.E.D. 
Theorem 9.14. Let $\left(\pi: \tilde{X} \rightarrow X, \Phi: \pi^{-1} \mathcal{E}_{\Lambda} \rightarrow \tilde{E}\right)$ be the blow up of the quantized contact manifold $(X, \mathcal{E})$ along a closed Lagrangian submanifold $\Lambda$. Let $\Re$ be

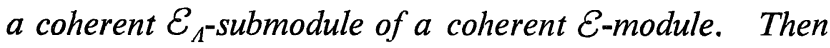

$$
\text { codim supp } \tilde{\mathcal{E}} \otimes_{\mathcal{E}_{\Lambda}} \mathfrak{\Omega} \geq \operatorname{codim} \text { supp } \mathscr{\Omega}
$$

Theorem 9.15. Let $(X, \mathcal{E})$ be a quantized logarithmic contact manifold and $\mathcal{A}$ a coherent sub $\mathcal{E}(0)$-algebra of $\mathcal{E}$ with zariskian fibers. Let $\Re$ be a coherent $\mathcal{A}$-submodule of a coherent $\mathcal{E}$-module. Then, for $j<d$,

$$
\text { codim supp } \Re \geq d \text { iff } \mathcal{E x t}_{\mathcal{A}}^{j}(\mathscr{H}, \mathcal{A})=0 \text {. }
$$

Proof of Theorem 9.14. Let $\Re$ be a coherent $\mathcal{E}_{\Lambda^{-}}$-submodule of a coherent $\mathcal{E}$-module. Suppose codim supp $\Re \geq d$. Then by Theorem 9.15 .

$$
\mathcal{E}_{x t_{\mathcal{E}_{\Lambda}}^{j}}\left(\mathscr{\Omega}, \mathcal{E}_{\Lambda}\right)=0, \text { for } j<d .
$$

Hence by Proposition 9.13.

$$
\mathcal{E} x t_{\pi^{-1} \mathcal{E}_{\Lambda}}^{j}\left(\pi^{-1} \mathcal{I}, \tilde{\mathcal{E}}\right)=0 \text { for } j<d .
$$

Therefore

$$
\mathcal{E x t}_{\mathcal{E}}^{j}(\tilde{\mathcal{E}} \otimes \Re, \tilde{\mathcal{E}})=0 \text { for } j<d
$$

Theorem 9.14 is now a consequence of $(9.15 .1)$ and Theorem 9.15. Q.E.D. Theorem 9.15 is a consequence of the two following Lemmas.

Lemma 9.16. Let $\Re$ be a coherent $\mathcal{A}$-submodule of a coherent $\mathcal{E}$-module. Then

(i) $\operatorname{Ext}_{\mathcal{A}}^{j}(\Re, \mathcal{A})=0$ iff $j<$ codim supp $\Re$.

(ii) codim supp $\mathcal{E x t}_{\mathcal{A}}^{j}(\Re, \mathcal{A}) \geq j$.

Lemma 9.17. Let $W$ be an irreducible component of the supports of a coherent $\mathcal{A}$-module $\Re$. If $W$ has codimension $d$ then

$$
W \subset \operatorname{supp} \operatorname{Ext}_{\AA}^{d}(\mathscr{\Omega}, \AA) \text {. }
$$

Proof of Theorem 9.15. If codim supp $\Re \geq d$ then, by 9.16 (i),

$$
\mathcal{E x t}_{\mathfrak{A}}^{j}(\Re, \mathscr{A})=0 \quad \text { if } j<d .
$$

Suppose that $\mathcal{E x t}^{j}(\mathscr{M}, \mathcal{A})=0$ for $j>d$ and let $W$ be an irreducible component of supp $\mathscr{M}$. By Lemma $9.17 W$ is contained in supp $\mathcal{E x t}_{\mathfrak{A}}^{j}(\mathscr{R}, \mathcal{A})$ for some 
$j \geq d$. Therefore, by 9.16 (ii), codim $V \geq d$.

Q.E.D.

Proof of Lemma 9.16. It is well known that

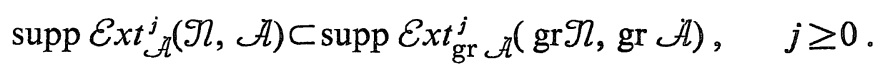

We notice that the morphism gr $A \rightarrow \mathcal{O}_{X}$ is flat. Actually gr $\mathcal{A} \rightarrow \mathrm{gr} \mathcal{E}$ is flat because for any $p \in X \operatorname{gr} \mathcal{E}_{p}$ is the localization of $\mathrm{gr} \mathcal{A}_{p}$ by any invertible homogeneous element of gr $\mathcal{E}$ of negative order. It is well known that gr $\mathcal{E} \rightarrow$ $\mathcal{O}_{X}$ is flat. Therefore

$$
\operatorname{supp} \mathcal{E x t}_{\mathscr{O}_{X}}^{j}\left(\mathcal{O}_{X} \otimes \operatorname{gr} \mathscr{M}, \mathcal{O}_{X}\right) \supset \operatorname{supp} \mathcal{E x t} t_{\mathcal{A}}^{j}(\mathscr{I}, \mathcal{A}) \text {. }
$$

Let $\mathscr{M}$ be a coherent $\mathcal{E}$-module that contains $\mathscr{R}$ as a $\mathcal{A}$-submodule. We can suppose that $\mathscr{N}$ generates $\mathscr{M}$. Then gr $\mathscr{I}$ generates gr $\mathscr{M}$ as a gr $\mathcal{E}$-module and

$$
\text { supp gr } \mathcal{E} \otimes_{\mathrm{gr} \mathcal{A}} \operatorname{gr} \mathscr{N}=\operatorname{supp} \text { gr } \mathscr{M}=\operatorname{supp} \mathscr{I} \text {. }
$$

Since the morphism $\mathrm{gr} \mathcal{E} \rightarrow \mathcal{O}_{X}$ is faithfully flat

$$
\operatorname{supp} \mathcal{O}_{X} \otimes_{\text {gr } \mathscr{A}} \operatorname{gr} \mathscr{I}=\operatorname{supp} \mathscr{I} \text {. }
$$

Statements (9.16.1) and (9.16.2) allow us to deduce Lemma 9.16 from the well known theorem of Analytic geometry we obtain when substituting $\mathcal{A}$ by $\mathcal{O}_{X}$ in the statement of 9.16 .

Q.E.D.

Proof of Lemma 9.17. Let $p^{0}$ be a generical point of $W$. If $j<d$ then $\mathcal{E} x t^{j}(\Re, \mathcal{A})=0$ by 9.16 (i). If $j>d$ then we can suppose by 9.16 (ii) that $\mathcal{E} x t^{j}(\mathscr{I}, \mathcal{A})$ vanishes at $p^{0}$. Suppose now that $\mathcal{E} x t^{d}(\mathscr{N}, \mathcal{A})_{p^{0}}$ equals 0 . Then

$$
\mathbb{R}_{\mathcal{H}}(\mathfrak{l}, \mathcal{A})_{p^{0}}=0 \text {. }
$$

Hence

$$
\Re_{p^{0}}=\mathbb{R} \operatorname{Ham}_{\mathcal{A}}\left(\boldsymbol{R} \operatorname{Ham}_{\mathcal{A}}(\mathscr{I}, \mathcal{A}), \mathcal{A}\right)_{p^{0}}=0 .
$$

The assumption above lead us into a contradiction and therefore there is an open dense subset of $W$ that is contained in supp $\mathcal{E x t}_{\mathcal{A}}^{d}(\Re, \mathcal{A})$.

This ends the proof of Lemma 9.17.

\section{\$10. Blow up of a Quantized Logarithmic Contact Manifold along Its Residual Submanifold}

Let $(X, \mathcal{L})$ be a logarithmic contact manifold. We will suppose in this section 
that the set of poles $Y$ of $(X, \mathcal{L})$ is smooth. Let $Z$ be the residual submanifold of $X$. Let

$$
\pi: \tilde{X}=\operatorname{Projan}\left(\oplus_{k \geq 0} I_{Z}^{k}\right) \rightarrow X
$$

be the blow up of $X$ along $Z$. Let $E$ be the exceptional divisor of $\pi$ and $\tilde{Y}$ the proper inverse image of $Y$.

Proposition 10.1. (i) The $\mathcal{O}_{\tilde{X}-\text { module }} \tilde{\mathcal{L}}=\pi^{*} \mathcal{L}$ is a structure of logarithmic contact manifold with poles along $\tilde{Y}$. Moreover $\mathcal{L}$ is the only structure of logarithmic contact manifold on $\tilde{X}$ for which $\pi$ is a morphism of logarithmic contact manifolds.

(ii) There is one and only one morphism of conic manifolds $\hat{\pi}: \hat{\tilde{X}} \rightarrow \hat{X}$ such that the following diagram commutes.

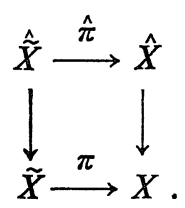

Moreover $\hat{\pi}$ is a morphism of homogeneous symplectic manifolds.

Definition 10.2. (i) We call the pair $(\pi: \tilde{X} \rightarrow X, \tilde{\mathcal{L}})$ the blow up of the contact manifold $(X, \mathcal{L})$ along its residual submanifold.

(ii) We call $\hat{\pi}: \hat{\tilde{X}} \rightarrow \hat{X}$ the blow up of the homogeneous logarithmic symplectic manifold $\hat{X}$ along its residual submanifold.

Remark 10.3. We notice that $\pi[\hat{\pi}]$ is a morphism of logarithmic contact manifolds [homogeneous logarithmic symplectic manifolds] but is not a local homeomorphism.

Proof of Proposition 10.1. (i) Since the problem is local in $X$ we can assume that $X$ is the affine logarithmic contact manifold $\left[d x_{n+1}-p_{1} d x_{1} / x_{1}-\right.$ $\left.\sum_{i=1}^{n} p_{i} d x_{i}\right]$. Then $\tilde{X}$ is the patching of the affine logarithmic contact manifolds

$$
\begin{aligned}
& X^{\prime}=\operatorname{Spec}\left(C\left[x_{1}, \cdots, x_{n+1}, \frac{p_{1}}{x_{1}}, p_{2}, \cdots, p_{n}\right]\right) \\
& X^{\prime \prime}=\operatorname{Spec}\left(C\left[\frac{x_{1}}{p_{1}}, x_{2}, \cdots, x_{n+1}, x_{n+1}-p_{1}, p_{2}, \cdots, p_{n}\right]\right) .
\end{aligned}
$$

and

Statement (i) follows from the equalities 


$$
\begin{aligned}
\left.\pi^{*} \omega\right|_{X^{\prime}} & =d x_{n+1}-\frac{p_{1}}{x_{1}} d x_{1}-\sum_{i=2}^{n} p_{i} d x_{i}, \\
\left.\pi^{*} \omega\right|_{x^{\prime \prime}} & =d\left(x_{n+1}-p_{1}\right)-p_{1} d \frac{x_{1}}{p_{1}} \mid \frac{x_{1}}{p_{1}}-\sum_{i=2}^{n} p_{i} d x_{i} . \\
x_{1}^{\prime} & =x_{1}, \quad x_{n+1}^{\prime}=x_{n+1}, \quad p_{1}^{\prime}=\frac{p_{1}}{x_{1}} \\
x_{1}^{\prime \prime} & =\frac{x_{1}}{p_{1}}, \quad x_{n+1}^{\prime \prime}=x_{n+1}-p_{1}, \quad p_{1}^{\prime \prime}=p_{1} .
\end{aligned}
$$

(ii) Put

The logarithmic contact manifold $\tilde{X}$ is the patching of the affine logarithmic contact manifolds

$$
\begin{aligned}
& X^{\prime}=\left[d x_{n+1}^{\prime}-p_{1}^{\prime} d x_{1}^{\prime}-\sum_{i=2}^{n} p_{i} d x_{i}\right] \\
& X^{\prime \prime}=\left[d x_{n+1}^{\prime \prime}-p_{1}^{\prime \prime} \frac{d x_{1}^{\prime \prime}}{x_{1}^{\prime \prime}}-\sum_{i=2}^{n} p_{i} d x_{i}\right]
\end{aligned}
$$

We notice that $\hat{X}=[\theta]_{n+1}, \hat{X}^{\prime}=\left[\theta^{\prime}\right]_{n+1}, \hat{X}^{\prime \prime}=\left[\theta^{\prime \prime}\right]_{n+1}$, where

$$
\begin{aligned}
& \theta=\sum_{i=1}^{n} \xi_{i} \delta x_{i}, \\
& \theta^{\prime}=\xi_{1}^{\prime} \delta x_{1}+\sum_{i=2}^{n} \xi_{i} d x_{i}+\sum \xi_{n+1} d x_{n+1}^{\prime}, \\
& \theta^{\prime \prime}=\xi_{1} \delta x_{1}^{\prime \prime}+\sum_{i=2}^{n} \xi_{i} d x_{i}+\xi_{n+1} d x_{n+1}^{\prime \prime} .
\end{aligned}
$$

Some simple computations show that

$$
\left.\theta^{\prime}\right|_{X^{\prime} \cap x^{\prime \prime}}=\left.\theta^{\prime \prime}\right|_{X^{\prime} \cap x^{\prime \prime}},\left.\quad \pi^{*} \theta\right|_{X^{\prime}}=\theta^{\prime},\left.\quad \pi^{*} \theta\right|_{X^{\prime \prime}}=\theta^{\prime \prime} . \quad \text { Q.E.D. }
$$

Remark 10.4. We notice that

$$
\tilde{Y}=\left\{x^{0} \in X^{\prime \prime}: \frac{x_{1}}{p_{1}}\left(x^{0}\right)=0\right\} \quad \text { and } \quad \tilde{X} \backslash \tilde{Y}=X^{\prime} .
$$

Theorem 10.5. Let $(X, \mathcal{E})$ be a quantized contact manifold with poles along a smooth divisor $Y$ of $X$. Then there is a quantization $\tilde{\mathcal{E}}$ of the blow up of the logarithmic contact manifold $X$ along its residual submanifold and a self dual morphism of self dual filtered $\boldsymbol{C}$-algebras

$$
\Phi: \pi^{-1} \mathcal{E} \rightarrow \tilde{\varepsilon}
$$

such that $\left.\Phi\right|_{\tilde{X} \backslash E}:\left.\left.\pi^{-1} \mathcal{E}\right|_{\tilde{X} \backslash E} \rightarrow \tilde{E}\right|_{\tilde{X} \backslash E}$ is an isomorphism and the right Ideal $\mathcal{G}_{-1 / 2} \tilde{E}$ of $\tilde{\mathcal{E}}$ is proper at least along $E$.

Moreover, the pair $(\Phi, \tilde{\mathcal{E}})$ is unique up to a unique isomorphism.

Definition 10.6. We call the pair $\left(\pi: \tilde{X} \rightarrow X, \Phi: \pi^{-1} \mathcal{E} \rightarrow \tilde{E}\right)$ the blow up of the quantized contact manifold $(X, \mathcal{E})$ along its residual set. 
Proof of Theorem 10.5. We will prove the Theorem in the case $(X, \mathcal{E})$ is the quantization of the affine logarithmic contact manifold (10.3.1). The generalization of this result is similar to (9.12) and therefore omited.

We patch the quantizations $\mathcal{E}^{\prime}, \mathcal{E}^{\prime \prime}$ of $X^{\prime}, X^{\prime \prime}$ by the self dual quantized contact transformation $\Psi: \mathcal{E}^{\prime} \rightarrow \mathcal{E}^{\prime \prime}$ determined by:

$$
\begin{aligned}
x_{1}^{\prime} & \mapsto \delta_{x_{1}^{\prime \prime}} x_{1}^{\prime \prime} \partial_{x_{n+1}^{\prime \prime}}^{-1} \\
\partial_{x_{1}^{\prime}} & \mapsto x_{1}^{\prime \prime-1} \partial_{x_{n+1}^{\prime \prime}} \\
x_{n+1}^{\prime} & \mapsto x_{n+1}^{\prime \prime}-\left(\delta_{x_{1}^{\prime \prime}}+\frac{1}{2}\right) \partial_{x_{n+1}}^{-1} \\
\partial_{x_{n+1}^{\prime}} & \mapsto \partial_{x_{n+1}^{\prime \prime}} .
\end{aligned}
$$

We introduce self dual quantized contact transformations

$$
\begin{aligned}
& \Phi^{\prime}:\left.\left.\pi^{-1} \mathcal{E}\right|_{X^{\prime} \backslash\left\{x_{1}^{\prime}=0\right\}} \rightarrow \tilde{\mathcal{E}}\right|_{X^{\prime} \backslash\left\{x_{1}^{\prime}=0\right\}}, \\
& \Phi^{\prime \prime}:\left.\left.\pi^{-1} \mathcal{E}\right|_{X^{\prime \prime} \backslash\left\{p_{1}^{\prime \prime}=0\right\}} \rightarrow \tilde{\mathcal{E}}\right|_{X^{\prime \prime} \backslash\left\{p_{1}^{\prime \prime}=0\right\}},
\end{aligned}
$$

determined respectively by:

$$
\begin{aligned}
& x_{1} \mapsto x_{1}^{\prime} \\
& x_{n+1} \mapsto x_{n+1}^{\prime} \\
& \delta_{x_{1}} \mapsto x_{1}^{\prime} \partial_{x_{1}^{\prime}} \\
& x_{1} \mapsto-\delta_{x_{1}^{\prime \prime}} x_{1}^{\prime \prime} \partial_{x_{n+1}^{\prime \prime}}^{-1} \\
& \partial_{x_{n+1}} \mapsto \partial_{x_{n+1}^{\prime}} \\
& x_{n+1} \mapsto x_{n+1}^{\prime \prime}-\left(\delta_{x_{1}^{\prime}}+\frac{1}{2}\right) \partial_{x_{n+1}^{\prime \prime}}^{-1} \\
& \delta_{x_{1}} \mapsto \delta_{x_{1}^{\prime \prime}} \\
& \partial_{x_{n+1}} \mapsto \partial_{x_{n+1}^{\prime \prime}}
\end{aligned}
$$

Just like in (9.10) there is one and only one morphism $\Phi: \pi^{-1} \mathcal{E} \mapsto \tilde{\mathcal{E}}$ that extends $\Phi^{\prime}$ and $\Phi^{\prime \prime}$.

We call the pair $(\pi: \tilde{X} \rightarrow X, \Phi: \mathcal{E} \rightarrow \tilde{E})$ the blow up of the affine logarithmic contact manifold $(X, \mathcal{E})$ along the residual ideal $\left(\delta_{x_{1}}\right)$.

Let $\tilde{\mathcal{E}}_{0}$ be another self dual quantization of $\tilde{X}$ and $\Phi_{0}: \pi^{-1} \mathcal{E} \rightarrow \tilde{\mathcal{E}}_{0}$ another morphism of self dual filtered $\boldsymbol{C}$-algebras such that $\left.\Phi_{0}\right|_{\tilde{X} \backslash E}:\left.\left.\pi^{-1} \mathcal{E}\right|_{\tilde{X} \backslash E} \rightarrow \tilde{\mathcal{E}}\right|_{\tilde{X} \backslash E}$ is an isomorphism and the right ideal $\left(\delta_{x_{1}}\right) \tilde{\mathcal{E}}_{0}$ is proper at least along $E$.

The $\boldsymbol{C}$-algebra $\tilde{\mathcal{E}}_{0}$ is the glueing of $\mathcal{E}^{\prime}$ and $\tilde{\mathcal{E}}^{\prime \prime}$ along some quantized contact transformation $\Psi_{0}$. Put $\Phi_{0}^{\prime}=\left.\Phi_{0}\right|_{X^{\prime}}, \Phi_{0}^{\prime \prime}=\left.\Phi_{0}\right|_{X^{\prime \prime}}$. Then there are unique microdifferential operators $A, B \in \tilde{\mathcal{E}}_{0}(0)\left(X^{\prime}\right)$ such that

$$
\Phi_{0}^{\prime}\left(\delta_{x_{1}}\right)=x_{1}^{\prime} \partial_{x_{1}^{\prime}}+x_{1}^{\prime} A+B,
$$

and $\left[\partial_{x_{1}^{\prime}}, B\right]=0$. Since $x_{1} \mathcal{E}^{\prime}$ is the maximal right ideal of $\mathcal{E}^{\prime}$ that is proper along $\left\{x_{1}=0\right\}$ then $B$ equals 0 . We define a quantization $\chi^{\prime}$ of $\mathrm{id}_{X^{\prime}}$ by 


$$
\begin{array}{llll}
x_{1} \mapsto \Phi_{0}^{\prime}\left(x_{1}\right), & \partial_{x_{1}} \mapsto \partial_{x_{1}^{\prime}}+A, & \\
x_{j} \mapsto \Phi_{0}^{\prime}\left(x_{j}\right), & \partial_{x_{j}^{\prime}} \mapsto \Phi_{0}^{\prime}\left(\partial_{x_{j}}\right), & 2 \leq j \leq n+1 .
\end{array}
$$

Since $\Phi_{0}^{\prime \prime}\left(x_{1}\right)$ belongs to the right ideal of $\mathcal{E}^{\prime \prime}$ generated by $\Phi_{0}^{\prime \prime}\left(\delta_{x_{1}}\right)$ there is a logarithmic microdifferential operator $A \in \mathcal{E}^{\prime \prime}\left(X^{\prime \prime}\right)$ such that

$$
\Phi_{0}^{\prime \prime}\left(x_{1}\right)=\Phi_{0}^{\prime \prime}\left(\delta_{x_{1}}\right) A
$$

We define a quantization $\chi^{\prime \prime}$ of $\mathrm{id}_{X^{\prime \prime}}$ by

$$
\begin{array}{rlrl}
x_{1}^{\prime \prime} & \mapsto-A \Phi_{0}^{\prime \prime}\left(\partial_{x_{n+1}}\right), & \delta_{x_{1}^{\prime \prime}} \mapsto \Phi_{0}^{\prime \prime}\left(\delta_{x_{1}}\right), & \\
x_{j}^{\prime \prime} & \mapsto \Phi_{0}^{\prime \prime}\left(x_{j}\right), & \partial_{x_{j}^{\prime \prime}} \mapsto \Phi_{0}^{\prime \prime}\left(\partial_{x_{j}}\right), & \\
x_{n+1}^{\prime \prime} & \mapsto \Phi_{0}^{\prime \prime}\left(\left(\delta_{x_{1}}+x_{n+1} \partial_{x_{n+1}}+\frac{1}{2}\right) \partial_{x_{n+1}}^{-1}\right), & \\
\partial_{x_{n+1}^{\prime \prime}} & \mapsto \Phi_{0}^{\prime \prime}\left(\partial_{x_{n+1}}\right) . & &
\end{array}
$$

We can patch the morphisms $\chi^{\prime}, \chi^{\prime \prime}$ into a morphism $\chi$ such that $\Psi \Phi=\Phi_{0}$.

Q.E.D.

\section{§11. Total Blow up of a Logarithmic Contact Manifold along a Lagrangian Submanifold}

Let $\left(X_{0}, \mathcal{E}_{0}\right)$ be a quantized logarithmic contact manifold with poles along a smooth divisor $Y$. We define a morphism of ringed spaces

$$
\left(\pi_{k 0}: X_{k} \rightarrow X_{0}, \Phi_{k 0}: \pi_{k 0}^{-1} \mathcal{E}_{0} \rightarrow \mathcal{E}_{k}\right)
$$

where $\left(X_{k}, \mathcal{E}_{k}\right)$ is a quantized logarithmic contact manifold with poles along a smooth divisor $Y_{k}$ inductively as follows. Put $\pi_{00}=\mathrm{id}_{X_{0}}, \Phi_{00}=\mathrm{id}_{\mathcal{E}_{0}}$. Given $(11.0 .1)_{k}, k \geq 0$, we define

$$
\left(\pi_{k+1, k}: X_{k+1} \rightarrow X_{k}, \Phi_{k+1, k}: \pi_{k+1, k}^{-1} \mathcal{E}_{k} \rightarrow \mathcal{E}_{k+1}\right)
$$

as the blow up of $\left(X_{k}, \mathcal{E}_{k}\right)$ along its residual set and put

$$
\pi_{k+1,0}=\pi_{k 0} \pi_{k+1, k}, \Phi_{k+1,0}=\Phi_{k+1, k} \Phi_{k 0}: \pi_{k+1}^{-1} \mathcal{E}_{0} \rightarrow \mathcal{E}_{k+1} .
$$

Definition 11.1. We call the pair $(11.0 .1)_{k}$ the $k$-blow up of the logarithmic contact manifold $\left(X_{0}, \mathcal{E}_{0}\right)$ along its residual set.

Let $(X, \mathcal{E})$ be a quantized contact manifold and $\Lambda$ a closed Lagrangian submanifold of $X$. Let $\left(\pi_{0}: X_{0} \rightarrow X, \Phi_{0}: \pi_{0}^{-1} \mathcal{E}_{\Lambda} \rightarrow \mathcal{E}_{0}\right)$ be the blow up of $(X, \mathcal{E})$ along 1 . Let $\left(\pi_{k 0}: X_{k} \rightarrow X_{0}, \Phi_{k 0}: \pi_{k 0}^{-1} \mathcal{E} \rightarrow \mathcal{E}_{k}\right)$ be the $k$-th blow up of $\left(X_{0}, \mathcal{E}_{0}\right)$ along its residual set. Define $\pi_{k}=\pi_{k 0} \pi_{k+1, k}, \Phi_{k}=\Phi_{k 0} \Phi_{0}$. 
There is a canonical immersion $X_{k} \backslash Y_{k} \hookrightarrow X_{k+1} \backslash Y_{k+1}$ such that the following diagram commutes.

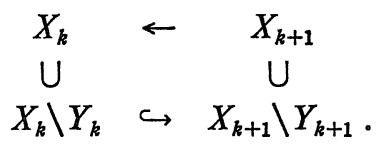

Put

$$
\tilde{X}=\underset{k}{\lim }\left(X_{k} \backslash Y_{k}\right) .
$$

The contact structures $\left.\mathcal{L}_{k}\right|_{X_{k} \backslash Y_{k}}$ define a contact structure $\tilde{\mathcal{L}}$ on $\tilde{X}$. The morphisms $\pi_{k}^{\prime}=\left.\pi_{k}\right|_{X_{k} \backslash Y_{k}}: X_{k} \backslash Y_{k} \rightarrow X$ define a morphism $\pi: \tilde{X} \rightarrow X$. The quantizations $\left.\mathcal{E}_{k}\right|_{X_{k} \backslash Y_{k}}$ of $X_{k} \backslash Y_{k}$ define a quantization $\tilde{\mathcal{E}}$ of $\tilde{X}$. The morphisms $\Phi_{k}^{\prime}=$ $\left.\Phi_{k}\right|_{X_{k} \backslash Y_{k}}$ define a morphism of self dual filtered $\boldsymbol{C}$-Algebras

$$
\Phi: \pi^{-1} \varepsilon_{\Lambda} \rightarrow \tilde{\varepsilon}
$$

Definition 11.2. We call the pair

$$
\left(\pi: \tilde{X} \rightarrow X, \Phi: \pi^{-1} \mathcal{E}_{\Lambda} \rightarrow \tilde{E}\right),
$$

the total blow up of the quantized contact manifold $(X, \mathcal{E})$ along the (closed) Lagrangian submanifold $\Lambda$.

Given a coherent $\mathcal{E}_{\Lambda}$-module $\eta$ we call the $\tilde{\mathcal{E}}$-module $\tilde{\mathcal{E}} \otimes_{\mathcal{E}_{\Lambda}} \Re$ the total blow up of $\Re$ along $\Lambda$.

Definition 11.3. Let $(X, \mathcal{E})$ be a quantized contact manifold. A coherent $\mathcal{E}$-module $\mathscr{M}$ is called holonomic if its support is a Lagrangian submanifold of $X$.

Theorem 11.4. Let $(X, \mathcal{E})$ be a quantized contact manifold and $\Lambda$ a closed Lagrangian submanifold of $X$. Let $\mathscr{M}$ be a holonomic $\mathcal{E}$-module and let $\mathscr{N}$ be a coherent sub $\mathcal{E}_{\Lambda}$-module of $\mathscr{M}$ such that $\mathscr{N}$ generates $\mathscr{M}$ as an $\mathcal{E}$-module and that locally there is a polynomial $b$ and a microdifferential operator $\vartheta$ verifying the following conditions.

(i) $\vartheta \in \mathcal{I}_{\Lambda}$.

(ii) $d \sigma(\vartheta) \equiv \theta \bmod I_{\Lambda} \Omega_{X}^{1}$.

(iii) $\sigma^{\prime}(\vartheta)+\frac{1}{2} \in I_{\Lambda}$.

(iv) $b(k) \neq 0, k=0,1,2, \cdots$.

(v) $b(\vartheta) \Re \subset \Re(-1)$.

Then $\tilde{\mathcal{E}} \otimes_{\mathcal{E}_{\Lambda}} \Re$ does not depend on the choice of $\Re$. 
Let $\left(X_{\omega \sigma}\right)$ be a covering of $X$ by copies of open sets of affine logarithmic contact manifolds. Put $\tilde{X}_{\alpha}=\pi^{-1}\left(X_{\alpha}\right)$. For each $\alpha$ there is a sub $\left.\varepsilon_{\Lambda}\right|_{X_{\alpha}}$-module $\Re_{\alpha}$ verifying the conditions of Theorem 11.4. This was proven by Kashiwara and Kawai in the regular holonomic case in [12] and generalized to holonomic systems by Laurent in [14]. We can patch the $\left.\tilde{\mathcal{E}}\right|_{\tilde{x}_{\alpha}}$-modules $\left.\tilde{\mathcal{E}}\right|_{\tilde{x}_{\infty}} \otimes_{\left.\mathcal{E}_{\Lambda}\right|_{x_{\alpha}}} \Re_{\infty}$ into a coherent $\tilde{\mathcal{E}}$-module $\tilde{\mathscr{M}}$.

Definition 11.5. We call the $\tilde{\mathcal{E}}$-module $\tilde{\mathscr{M}}$ the total blow up of the holonomic $\mathcal{E}$-module $\mathscr{M}$ along $\Lambda$.

Theorem 11.6. The $\tilde{\mathcal{E}}$-module $\tilde{\mathscr{M}}$ is holonomic.

In order to prove Theorems 11.4 and 11.6 we will first introduce a family of subrings of $\mathcal{E}$. Let $A$ be a closed Lagrangian submanifold of $X$. For any integer $k \geq 0$ let $\mathcal{A}_{(k)}$ be the subring of $\mathcal{E}$ locally generated by $\mathcal{E}_{\Lambda}$ and $\mathcal{E}(1) \mho^{k}$, where $\vartheta$ is a microdifferential operator verifying the conditions (i), (ii), (iii) of Theorem 11.4. The $\boldsymbol{C}$-Algebra $\mathcal{A}_{(k)}$ is noetherian, with zariskian fibers and self dual (cf. 11.11). Theorems 11.4 and 11.6 are a consequence of the following Lemmas.

Lemma 11.7. For any integer $k \geq 0$ there is one and only one morphism from $\left.\pi_{k}^{-1} \mathcal{A}_{(k)}\right|_{X_{k} \backslash Y_{k}}$ into $\left.\tilde{\mathcal{E}}\right|_{X_{k} \backslash Y_{k}}$ that extends the morphism $\Phi_{k}^{\prime}$ introduced before Definition 11.2.

We will still denote this morphism by $\Phi_{k}^{\prime}$.

Lemma 11.8. The morphisms

$$
\Phi_{k}^{\prime}:\left.\left.\pi_{k}^{-1} \mathcal{A}_{(k)}\right|_{X_{k} \backslash Y_{k}} \rightarrow \tilde{\mathcal{E}}\right|_{X_{k} \backslash Y_{k}}
$$

are flat for any non negative integer $k$.

Lemma 11.9. Let $\mathcal{M}$ be a holonomic $\mathcal{E}$-module and $\mathscr{N}$ a $\mathcal{E}_{\Lambda}$-submodule of $\mathscr{M}$ verifying the hipoteses of Theorem 11.4. Then $\mathscr{A}_{k} \otimes_{\mathcal{E}_{\Lambda}} \mathfrak{I}$ is isomorphic to $\mathscr{M}$.

Proof of Theorem 11.4. If $\mathscr{M}$ and $\mathscr{N}$ verify the hypothesis of the Theorem then

$$
\begin{aligned}
\left.\mathcal{E}\right|_{X_{k} \backslash Y_{k}} \otimes_{\mathcal{E}_{\Lambda}} \mathscr{I} & =\left.\mathcal{E}\right|_{X_{k} \backslash Y_{k}} \otimes_{\mathcal{A}_{(k)}} \mathscr{A}_{(k)} \otimes_{\mathcal{E}_{\Lambda}} \mathscr{I} \\
& =\left.\mathcal{E}\right|_{Y_{k} \backslash Y_{k}} \otimes_{\mathcal{H}_{(k)}} \mathscr{M} .
\end{aligned}
$$

Proof of Theorem 11.6. Let $\mathscr{M}$ be an holonomic $\mathcal{E}$-module. Then 
$\left.\tilde{\mathscr{M}}\right|_{X_{k} \backslash Y_{k}}$ equals $\left.\mathcal{E}_{k}\right|_{X_{k} \backslash Y_{k}} \otimes_{\mathcal{H}_{(k)}} \mathscr{M}$. Since the morphism

$$
\left.\mathcal{A}_{(k)} \rightarrow \mathcal{E}_{k}\right|_{X_{k} \backslash Y_{k}}
$$

is flat we conclude by a reasoning similar to the one used in the proof of Theorem 9.14 that

$$
\text { codim supp }\left(\left.\tilde{\mathscr{M}}\right|_{X_{k} \backslash Y_{k}}\right) \geq \operatorname{codim} \operatorname{supp} \mathscr{M} \text {. }
$$

Q.E.D.

Proof of Lemma 11.7. The uniqueness is obvious. Therefore we can suppose that $(X, \mathcal{E})$ equals the quantization of the affine logarithmic contact manifold of dimension $2 n+1$ and $\Lambda$ equals $\left\{x_{1}=\cdots=x_{n+1}=0\right\}$.

11.10. We will first study the $k$-th blow up of the quantization of the affine logarithmic contact manifold

$$
X_{0}=\left[d y_{n+1}-q_{1} \frac{d y_{1}}{y_{1}}-\sum_{i=2}^{n} q_{i} d y_{i}\right] .
$$

We notice that $X_{k}$ is the patching of $k$ affine logarithmic contact manifolds $X_{1}^{\prime}, \cdots, X_{k}^{\prime}$ and an affine logarithmic contact manifold $X_{k}^{\prime \prime}$ isomorphic to $X_{0}$. Actually suppose that the statement above is true. Then $X_{k+1}$ is the patching of $X_{1}^{\prime}, \cdots, X_{k}^{\prime},\left(X_{k}^{\prime \prime}\right)^{\prime},\left(X_{k}^{\prime \prime}\right)^{\prime \prime}$ and by the proof of Proposition 10.1. $\left(X_{k}^{\prime \prime}\right)^{\prime}$ is an affine contact manifold and $\left(X_{k}^{\prime \prime}\right)^{\prime \prime}$ is isomorphic to $X_{0}$. We put $X_{k+1}^{\prime}=$ $\left(X_{k}^{\prime \prime}\right)^{\prime}$ and $X_{k+1}^{\prime \prime}=\left(X_{k}^{\prime \prime}\right)^{\prime \prime}$. We introduce global sections $x_{k 1}, x_{k, n+1}, p_{k}$ of $X_{k}^{\prime}$, $y_{k 1}, y_{k 1}, q_{k 1}$ of $X_{k}^{\prime \prime}$ inductively as follows:

$$
\begin{array}{lll}
x_{k+1,1}=y_{k 1}, & x_{k+1, n+1}=y_{k, n+1}, & p_{k+1,1}=\frac{q_{k 1}}{x_{1}}, \\
y_{k+1,1}=\frac{y_{k 1}}{q_{k 1}}, & y_{k+1, n+1}=y_{k, n+1}-q_{k 1}, & q_{k+1,1}=q_{k 1} .
\end{array}
$$

We notice that, for any non negative integer $k$,

$$
\begin{aligned}
& X_{k}^{\prime}=\left[d x_{k, n+1}-p_{k 1} d x_{k 1}-\sum_{i=2}^{n} q_{i} d y_{i}\right], \\
& X_{k}^{\prime \prime}=\left[d y_{k, n+1}-q_{k 1} \frac{d y_{k 1}}{y_{k 1}}-\sum_{i=2}^{n} q_{i} d y_{i}\right] .
\end{aligned}
$$

Then the morphisms $\Phi_{k 0}^{\prime}=\left.\Phi_{k 0}\right|_{X_{j}^{\prime}}: \pi_{k}^{-1} \mathcal{E}_{0}\left|X_{j}^{\prime} \rightarrow \mathcal{E}_{k}\right|_{X_{j}^{\prime}}, \quad 1 \leq j \leq k$,

$$
\Phi_{k}^{\prime \prime}=\left.\Phi_{k 0}\right|_{X_{k}^{\prime \prime}}:\left.\left.\pi_{k}^{-1} \mathcal{E}_{0}\right|_{X_{k}^{\prime \prime}} \rightarrow \mathcal{E}_{k}\right|_{X_{k}^{\prime \prime}}
$$

are determined respectively by: 


$$
\begin{aligned}
& y_{1} \mapsto\left(-x_{j 1} \partial_{x_{j 1}}\right)^{j-1} x_{j 1} \partial_{x_{j, n+1}}^{1-j}, \quad \delta_{y 1} \mapsto x_{j 1} \partial_{x_{j 1}}, \\
& y_{n+1} \mapsto x_{j, n+1}-j\left(x_{j} \partial_{x_{j}}+\frac{1}{2}\right) \partial_{x_{j, n+1}}^{-1}, \quad \partial_{y_{n+1}} \mapsto \partial_{x_{j, n+1}} \text {, } \\
& x_{i} \mapsto x_{i}, \quad \partial_{x_{i}} \mapsto \partial_{x_{i}}, \quad 2 \leq i \leq n . \\
& y_{1} \mapsto\left(-\delta_{y_{k 1}}\right)^{k} y_{k 1} \partial_{y_{k, n+1}}^{-k}, \quad \delta_{y_{1}} \mapsto \delta_{y_{k 1}}, \\
& y_{n+1} \mapsto y_{k, n+1}-k\left(\delta_{y_{k 1}}+\frac{1}{2}\right) \partial_{y_{k_{0} n+1}}, \quad \partial_{y_{n+1}} \mapsto \partial_{y_{k_{0} n+1}} \text {, } \\
& y_{i} \mapsto y_{i}, \quad \partial_{y_{i}} \mapsto \partial_{y_{i}}, \quad 2 \leq i \leq n .
\end{aligned}
$$

11.11. We will now finish the proof of Lemma 11.7. Let $X$ denote the affine contact manifold

$$
\left[d x_{n+1}-\sum_{i=1}^{n} p_{i} d x_{i}\right]
$$

and $\Lambda$ its submanifold $\left\{x_{1}=\cdots=x_{n+1}=0\right\}$.

Notice that $\vartheta$ is determined modulo $\mathcal{G}_{\Lambda}(-1)$. Therefore $\vartheta^{k}$ is determined modulo $\mathcal{E}_{1}(-1)$ and the sub $\mathcal{E}(0)$-algebra of $\mathcal{E}$ generated by $\mathcal{E}_{1}$ and $\mathcal{E}_{(1)} \vartheta^{k}$ does not depend of the choice of $\vartheta$. We can choose

$$
\vartheta=\sum_{i=1}^{n+1} x_{i} \partial_{x_{i}}+\frac{n}{2}
$$

Notice that $\left(\partial_{x_{n+1}} \vartheta^{k}\right)^{*}=(-)^{k+1} \partial_{x_{n+1}} \vartheta^{k}$. Since $\mathcal{E}_{A}$ is self adjoint the $\mathcal{E}_{\Lambda^{-} \text {-module }}$ generated by $\mathcal{E}_{\Lambda}$ and $\mathcal{E}_{(1)}$ and $\mathcal{E}_{(1)} \vartheta^{k}$ is also self adjoint.

We will denote by $\bar{X}$ the blow up of $X$ along $\Lambda$ and by $\bar{X}_{1}, \cdots, \bar{X}_{n+1}$ the affine logarithmic contact manifolds introduced in the proof of Theorem 9.5. Then the morphism

$$
\Phi_{k}^{\prime}:\left.\left.\pi_{k}^{-1} \mathcal{E}_{\Lambda}\right|_{X_{k} \backslash Y_{k}} \rightarrow \mathcal{E}\right|_{X_{k} \backslash Y_{k}}
$$

is the patching of its restrictions

$$
\Phi_{k l}^{\prime}=\left.\Phi_{k}^{\prime}\right|_{x_{k l}}, \quad 1 \leq l \leq n,
$$

where $X_{k l}=\pi_{k}^{-1}\left(\bar{X}_{l}\right) \cap\left(X_{k} \backslash Y_{k}\right)$. We can suppose $l=1$. We will identify $\bar{X}_{1}$ and $X_{0}=\left[d y_{n+1}-p_{1} d y_{1}-\sum_{i=2}^{n} p_{i} d y_{i}\right]$. Then

$$
\begin{array}{cc}
\left.\Phi_{k 1}^{\prime}(\vartheta)\right|_{X_{s 1}}=x_{s 1} \partial_{x_{s 1}}, & 1 \leq s \leq k, \\
\left.\Phi_{k 1}^{\prime}\left(\partial_{x_{n+1}}\right)\right|_{x_{s 1}}=x_{s 1}^{-1}\left(x_{s 1} \partial_{x_{s 1}}\right)^{1-s} \partial_{x_{s, n+1}}^{s} . &
\end{array}
$$

Therefore

$$
\left.\Phi_{k 1}^{\prime}\left(\partial_{x_{n+1}} \vartheta^{k}\right)\right|_{X_{s 1}}=\partial_{x_{s 1}}\left(x_{s 1} \partial_{x_{s 1}}\right)^{k-s} \partial_{x_{s, n+1}} .
$$

This ends the proof of Lemma 11.7.

Proof of Lemma 11.8. The proof of this Lemma is very similar to the proof 
of Proposition 9.13. Therefore we will only prove the following fact.

Fact 11.12. Put

$$
\begin{aligned}
& A=\boldsymbol{C}\left[x_{1}, \cdots, x_{n+1}, p_{1}, \cdots, p_{n}, \boldsymbol{\xi}, \boldsymbol{\xi}^{-1}\right], \\
& B=\boldsymbol{C}\left[y_{1}, \cdots, y_{n+1}, q_{1}, \cdots, q_{n}, \eta, \eta^{-1}\right] .
\end{aligned}
$$

For $k \geq 1$ let $A_{k}$ be the subring of $A$ generated by

$$
x_{j} \xi, 1 \leq j \leq n+1, \quad p_{j}, 1 \leq j \leq n, \quad z^{k} \xi^{k+1}, \xi^{-1},
$$

where $z=x_{n+1}-\sum_{i=1}^{n} x_{i} p_{i}$. For $k \geq 1$ let $\Psi_{k}: A_{k} \rightarrow B$ be the morphism of $\boldsymbol{C}$ algebras defined by:

$$
\begin{array}{rlrlrl}
x_{1} \xi & \mapsto \eta, & x_{j} \xi & \mapsto y_{j} \eta, & & 2 \leq j \leq n+1, \\
z & \mapsto y_{1}^{k+1} q_{1}^{k}, & p_{j} \mapsto q_{j}, & 2 \leq j \leq n+1, \\
\xi^{-1} & \mapsto y_{1}^{k} q_{1}^{k-1} \eta^{-1} . & & &
\end{array}
$$

Then $\Psi_{k}$ is a flat morphism.

Proof. Let $A_{k}^{\prime}$ be the localization of $A_{k}$ by $x_{1} \xi$. Then $\Psi_{k}$ extends to a morphism $\Psi_{k}^{\prime}: A_{k}^{\prime} \rightarrow B$ and

$$
\begin{array}{rlrl}
\left(x_{1} \xi\right)^{-1} & \mapsto \eta^{-1}, & \left(x_{j} \xi\right)\left(x_{1} \xi\right)^{-1} \mapsto y_{j}, \quad 2 \leq j \leq n, \\
z^{k} \xi^{k+1}\left(x_{1} \xi\right)^{-(k+1)} & \mapsto q_{1} \\
\Psi_{1}\left(x_{1}\right) & =y_{1} .
\end{array}
$$

Therefore $\Psi_{1}^{\prime}$ is an isomorphism.

For $k \geq 2$ let $A_{k}^{\prime \prime}$ be the localization of $A_{k}^{\prime}$ by $z^{k-1} \xi^{k}\left(x_{1} \xi\right)^{-k}$. Then $\Psi_{k}^{\prime}$ extends to a morphism $\Psi_{k}^{\prime \prime}: A_{k}^{\prime \prime} \rightarrow B$ and

$$
\left(z^{k-1} \xi^{k}\left(x_{1} \xi\right)^{-k}\right)^{-1} \mapsto y_{1} .
$$

Therefore $\Psi_{k}^{\prime \prime}$ is an isomorphism.

This ends the proof of Lemma 11.8.

Proof of Lemma 11.9. We will show that the morphisms (11.12.1) are isomorphisms.

$$
\begin{gathered}
\mathscr{A}_{(k)} \otimes_{\mathcal{E}_{\Lambda}} \mathscr{I} \rightarrow \mathscr{M} \\
P \otimes u \mapsto P u .
\end{gathered}
$$

Put $\mathcal{E}_{\Lambda}(l)=\mathcal{E}(l) \mathcal{E}_{\Lambda}=\mathcal{E}_{\Lambda} \mathcal{E}(l), \mathscr{\eta}(l)=\mathcal{E}(l) \mathscr{I}$. Then the sequence

$$
0 \rightarrow \mathscr{R}_{(l)} / \mathscr{R}_{(l-1)} \stackrel{\vartheta}{\rightarrow} \mathscr{\eta}_{(l)} / \mathscr{I}_{(l-1)} \rightarrow 0
$$




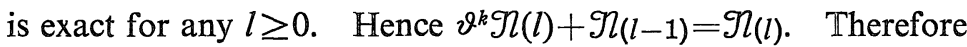

$$
\varepsilon_{(1)} \vartheta^{k} \Re_{(l)}+\Re_{(l)}=\Re_{(l+1)} \text {. }
$$

Henceforth $\Re_{(l)} \subset \mathcal{A}_{(k)} \Re$ for any integer $l$.

We will now show that (11.12.1) is a monomorphism. Let $\mathcal{A}_{(k), N}$ be the right $\operatorname{sub} \varepsilon_{A}$-module generated by $\left(\partial_{y} \vartheta^{k}\right)^{\nu}, 1 \leq \nu \leq N$. We will now show by induction in $N$ that the sequence (11.12.2) is exact.

$$
0 \rightarrow \mathcal{A}_{(k), N} \otimes_{\mathcal{E}_{1}} \mathscr{I} \rightarrow \mathscr{M}
$$

Suppose $N \geq 1$. We will show that

$$
\text { if } u \in \mathscr{M},\left(\partial_{y} \vartheta^{k}\right)^{N} u \in \mathscr{A}_{(k), N-1} \text { then } u \in \Re_{(-1)} \text {. }
$$

Actually if the hypothesis of (11.12.3) is satisfied then $\left(\partial_{y} \vartheta^{k}\right)^{N} \in \mathscr{l}(N-1)$. Therefore

$$
((\vartheta-N+1) \cdots(\vartheta-2)(\vartheta-1) \vartheta)^{k} u \in \mathscr{L}(-1) .
$$

Hence $u \in \mathscr{R}(-1)$.

Given sections $u_{1}, \cdots, u_{n}$ of $\mathscr{I}$ if $\sum_{k=0}^{N}\left(\partial_{y} \vartheta^{k}\right)^{j} \otimes u_{j}$ is mapped into 0 then $u_{N} \in \mathscr{L}(-1)$ by (11.12.3). Taking $v=\partial_{x} u_{N}$

$$
\left(\partial_{y} \vartheta^{k}\right)^{N} \otimes u_{N} \text { equals }\left(\partial_{y} \vartheta^{k}\right)^{N} \partial_{y}^{-1} \otimes v .
$$

Therefore by the induction hypothesis

$$
\sum_{j=0}^{N}\left(\partial_{y} \vartheta^{k}\right)^{j} \otimes u_{j}=0
$$

Propositionl11.13. Let $M$ be a complex manifold and $\lambda$ a point of $M$. Let $\tilde{M}$ be the blow up of $M$ along $\{\lambda\}$ and let $E$ be its exceptional divisor. Put $X=\mathbb{P}^{*} M$ and $\Lambda=\pi^{-1}(\lambda)$. Then. with the notations introduced in the begining of this chapter, there is a canonical isomorphism

$$
X_{1} \backslash Y_{1} \underset{\rightarrow}{\longrightarrow} \tilde{M} \backslash \mathbb{P}_{E}^{*} \tilde{M} .
$$

Moreover the canonical morphism from $\mathbb{P}^{*} \tilde{M} \backslash \mathbb{P}_{E}^{*} \tilde{M}$ into $\mathbb{P}^{*} M$ equals the canonical morphism from $X_{1} \backslash Y_{1}$ into $X$.

Proof. There are canonical open immersions of $\mathbb{P}^{*}(\tilde{X} \backslash E)$ into $X_{1} \backslash Y_{1}$ and $\mathbb{P}^{*} \tilde{M} \backslash \mathbb{P}_{E}^{*} \tilde{M}$. Therefore there is at most a morphism from $X_{1} \backslash Y_{1}$ into $\mathbb{P}^{*} \tilde{M} \backslash \mathbb{P}_{E}^{*} \tilde{M}$. Hence it is enough to prove locally the existence of the isomorphism. This can be easily done using local coordinates. We can prove the second statement in a similar way.

Q.E.D. 


\section{Acknowledgment}

I would like to express my gratitude towards my thesis adviser M. Kashiwara for suggesting this problem as well as for his advice and criticism duering the realization of this work.

\section{Bibliography}

[1] Bourbaki, N., Commutative Algebra, Herman

[2] Deligne, P., Equations differentielles a points singuliers reguliers, Springer Lect. Notes, 163 (1970).

[3] Grothendieck, A. and Dieudonné, J., Eléments de Géométrie Algébrique, I Springer Verlag, II Publ. Math. IHES, 4 (1960).

[4] Grothendieck, A., Techniques de construction en Géometrie analytic, Seminaire Henri Cartan, 13 ${ }^{e}$ année 1960/61 W.A. Benjamin (1967).

[5] Hartshorne, R., Algebraic Geometry, Springer Verlag, 1977.

[6] Hörmander, L., The analysis of linear partial differential operators III, Chapter XXI, Springer Verlag, 1985.

[7] Hironaka, H., Resolution of singularities of an algebraic variety over a field of characteristic 0, Ann. of Math., 79 (1964).

[8] Houzel, C., Géometrie analytic locale, Seminaire Henri Cartan, $13^{e}$ année, 1960/61, W.A. Benjamin (1967).

[9] Kashiwara, M., Introduction to Microlocal Analysis, Monographie $\mathrm{N}^{\circ} 32$ de L'Enseignement Mathématique (1986).

[10] Kashiwara, M. and Fernandes T.M., Involutivité des varietés microcharacteristiques, Bull. Soc. Math. France, 114 (1986), 393-402.

[11] Kashiwara, M. and Kawai, T., On Holonomic systems of Microdifferential Equations, III Systems with Regular Singularities, Publ. RIMS, Kyoto Univ., 17 (1981), 813-979.

[12] - Second microlocalization and asymptotic expansions, Springer Lect. Notes in Physics, 126 (1980), 21-76.

[13] Kashiwara, M. and Oshima, T., Systems of differential equations with regular singularities and their boundary value problems, Ann. of Maths., 106 (1977), 145-200.

[14] Laurent, Y., Poligone de Newton et $b$-fonctions pour les modules microdifferentielles, Ann. scient. Éc. Norm. Sup., $4^{e}$ série, 20 (1987), 391-441.

[15] Schapira, P., Microdifferential systerns in the Complex Domain, Springer-Verlag, 1985.

[SKK] Sato, M., Kawai, T. and Kashiwara, M.. Microfunctions and Pseudo-differential Equations, Springer Lect. Notes, 287 (1973), 265-529. 
